\title{
THE LOWER PART OF THE SPECTRUM OF THE HAMILTONIAN OF THE SPINLESS PAULI-FIERZ MODEL (A TWO-COMPONENT BOSE FIELD INTERACTING WITH A CHARGED PARTICLE)
}

\author{
R. A. MINLOS
}

\begin{abstract}
We investigate a model of a vector massive spinless Bose field in the space $R^{3}$ interacting with a non-relativistic particle, where the interaction parameter is assumed to be sufficiently small. We study the ground state of the Hamiltonian $H_{P}$ for a fixed total momentum $P$ of the system and show that such a state is nondegenerate and exists only for a bounded domain of values of this momentum. We also show that, apart from the ground state, the operator $H_{P}$ has no other eigenvalues below the continuous spectrum. Furthermore, the next two, "one-boson", branches of the spectrum of $H_{P}$ are constructed, which describe the scattering of one boson (with two possible polarization values) on the ground state.
\end{abstract}

\section{IntRoduction AND THE STATEMENT OF THE MAIN RESULTS}

In this paper we consider a model of a vector massive Bose field in the space $R^{3}$ interacting with a non-relativistic charged particle for a small value of the charge of the particle. Here we study the ground state of the Hamiltonian $H_{P}$ of the model for a fixed value of the total momentum $P$ of the system, as well as the branches of the continuous spectrum of the operator $H_{P}$ next in magnitude, which describe the scattering states of one boson (with various polarization values) on the ground state.

Although the whole area involved in studying the spectrum of Hamiltonians describing the interaction of one (or several) particles with an electromagnetic (vector or scalar) field has a long history (see [10, 19]), it has been very intensively developed in recent years (see 1, 2, 4, 5, 9, 11, 12, 13, 15, 16, 17, 18, 20, 22, 25]; there are detailed lists of references on this subject in 23 and in the book [25, 3]). The main results in these papers concern the construction of the ground state of the Hamiltonian $H_{P}$ and an investigation of its properties, under various assumptions and by various methods; the principal requirement remains the assumption that the constant of the interaction between the particle and the field is small. It turns out that when the dimension $d \geq 3$, a ground state exists only for a bounded domain $G_{0} \subset R^{d}$ of values of the momentum $P$ and disappears for $P \notin G_{0}$; in the case $d=1,2$, a ground state exists for all $P$. This state is non-degenerate in the case of a spinless model, and is doubly degenerate for a model with spin [17.

2000 Mathematics Subject Classification. Primary 81Q10; Secondary 47A10, 47A40, 47A55, 81T10, 81U99, 81V10.

Key words and phrases. Non-relativistic charged particle, Bose field, Pauli-Fierz model, Hamiltonian, spectrum, ground state, scattering.

The author thanks Professor H. Spohn and Dr. E. A. Zhizhina for useful discussions on questions relating to this paper. The author thanks the Mathematics Centre of the Munich Technical University, where the plan for this research emerged for the first time, for its warm hospitality and financial support. The author also thanks the following organisations for financial support: the Russian Foundation for Basic Research (grant \# 05-01-00449), the President Foundation for Support of Scientific Schools of Russia, and the American Foundation CRDF, grant RUM1-2603-MO-05. 
Using visual representations common in physics, a ground state of the Hamiltonian $H_{P}$ can be thought of as a charged particle surrounded by a "cloud" of a large number of bosons. The next question is how to describe (and construct) the state of this "cloud" when it has "emitted" (or "absorbed") one boson (or several bosons). Such states "scattering states" - belong to the space of the continuous spectrum of the operator $H_{P}$. The main part of this paper is devoted to such states when one boson has been emitted. At the same time, we find that the Hamiltonian $H_{P}$ has no "excited" levels, that is, the only eigenvalue of $H_{P}$ lying below the continuous spectrum is the energy of the ground state (if it exists).

Note that in the paper [12] mentioned above, branches of the continuous spectrum corresponding to scattering of one or several bosons on the ground state are also constructed for the case of a scalar boson field. This construction (after the ground state is obtained) is achieved by the methods of scattering theory.

This paper is a continuation of the papers [22] and [1, where the results discussed above were obtained for the case of a scalar field with linear interaction terms in the Hamiltonian $H_{P}$ (with respect to the creation and annihilation operators). In many places in this paper we actually repeat the steps in the construction used in these papers and we refer the reader to them for the proofs of the corresponding assertions.

The plan of the paper is as follows.

- In $\S 1$ we give a detailed description of the model and state the main results of the paper.

- In $\S 2$, so that the reader does not get bogged down in the subtleties of the analysis, we expound the main idea and the general plan of the construction developed in the paper.

- In $\S 3$ we do the main technical step in our constructions: when solving the equations defining the resolvent of the operator $H_{P}$ in the Fock space, we eliminate the higher components of the desired vector by expressing them in a special way via its lower components. After that the problem reduces to studying a family of Friedrichs-type operators.

- In $\S 4$ we describe the requisite properties of such operators and find the form of their resolvents and eigenfunctions.

- Finally, in $\S 5$, using the facts obtained in $\S 4$, we construct a ground state and lower branches of the continuous spectrum of the operator $H_{P}$.

We now turn to a systematic exposition of the paper.

The Hilbert space $\mathcal{H}$ of states of the model is

$$
\mathcal{H}=L_{2}\left(R^{d}\right) \otimes \mathcal{F}_{\text {sym }} \otimes \mathcal{F}_{\text {sym }},
$$

where the first factor $L_{2}\left(R^{d}\right)$ describes the state of the particle, and $\mathcal{F}_{\text {sym }} \otimes \mathcal{F}_{\text {sym }}=\mathcal{F}_{\text {sym }}^{\otimes 2}$ is the space of states of the boson field consisting of two components with different polarization; $\mathcal{F}_{\text {sym }}=\mathcal{F}_{\text {sym }}\left(L_{2}\left(R^{d}\right)\right)$ is the symmetric Fock space over the space $L_{2}\left(R^{d}\right)$ (see [21]).

The Hamiltonian of the model has the form (see [25, 3])

$$
H=\frac{1}{2}\left(p \otimes \mathbb{I}_{f}-e \mathbb{I}_{p} \otimes \mathcal{A}_{\varphi}(x)\right)^{2}+\mathbb{I}_{p} \otimes \mathcal{H}_{f}
$$

Here $p=-i \nabla_{x}$ is the particle momentum operator acting in $L_{2}\left(R^{d}\right), e$ is the charge of the particle, the $\mathcal{A}_{\varphi}(x), x \in R^{d}$, are the field operators, which have the form

$$
\mathcal{A}_{\varphi}(x)=\sum_{j=1,2} \int \frac{\varphi(k)}{\sqrt{2 \omega(k)}} e_{j}(k)\left(a_{j}^{*}(k) e^{-i(k, x)}+a_{j}(k) e^{i(k, x)}\right) d k, \quad x \in R^{d} .
$$


In formula (1.2), for each $k \in R^{d}$, the vectors $e_{j}(k), j=1,2$, are two orthonormal vectors that are orthogonal to the vector $k$ :

$$
\left(e_{j}(k), k\right)=0, \quad k \in R^{d}, \quad\left(e_{i}(k), e_{j}(k)\right)=\delta_{i j} .
$$

These vectors describe the polarization of the boson. Next, $\varphi(k)$ is a bounded smooth function, which is rapidly decreasing at infinity (a form factor); $\omega(k)$ is the dispersion of a free boson and is given by

$$
\omega(k)=\sqrt{k^{2}+m^{2}}+c_{0}, \quad m \geq 0, c_{0}>0 .
$$

Note that this function satisfies the inequality

$$
\omega\left(k_{1}\right)+\omega\left(k_{2}\right) \geq \omega\left(k_{1}+k_{2}\right)+c_{0}
$$

for any $k_{1}, k_{2} \in R^{d}$. The operators $a_{j}(k), a_{j}^{*}(k)$ are the operators of creation and annihilation of bosons (with polarization $j$ ) acting in the corresponding Fock space and obeying the following well-known commutation relations (see, for example, 21]):

$$
\left[a_{j_{1}}^{*}, a_{j_{2}}\right]=-\delta\left(k_{1}-k_{2}\right) \delta_{j_{1}, j_{2}}, \quad\left[a_{j_{1}}\left(k_{1}\right), a_{j_{2}}\left(k_{2}\right)\right]=\left[a_{j_{1}}^{*}\left(k_{1}\right), a_{j_{2}}^{*}\left(k_{2}\right)\right]=0 .
$$

Next, the operator $H_{f}$ in formula (1.1) is equal to

$$
H_{f}=\sum_{j=1,2} \int \omega(k) a_{j}^{*}(k) a_{j}(k) d k
$$

and acts in the space $\mathcal{F}_{\text {sym }} \otimes \mathcal{F}_{\text {sym }}$. Finally, $\mathbb{I}_{p}$ and $\mathbb{I}_{f}$ are the identity operators in the spaces $L_{2}\left(R^{d}\right)$ and $\mathcal{F}_{\text {sym }} \otimes \mathcal{F}_{\text {sym }}$, respectively.

Note that by virtue of the translational invariance of the system, its total momentum operator

$$
P=p \otimes \mathbb{I}_{f}+\mathbb{I}_{p} \otimes P_{f}
$$

commutes with the Hamiltonian $H$. Here $P_{f}$ is the field momentum operator

$$
P_{f}=\sum_{j=1,2} \int k a_{j}^{*}(k) a_{j}(k) d k .
$$

Hence the space $\mathcal{H}$ can be decomposed into the direct integral

$$
\mathcal{H}=\int \mathcal{H}_{P} d P
$$

of the Hilbert spaces $\left\{\mathcal{H}_{P}, P \in R^{d}\right\}$ ("eigenspaces" for the operator $P$ ), and the Hamiltonian $H$ into the direct integral

$$
H=\int H_{P} d P
$$

of operators, each acting in $\mathcal{H}_{P}$ (see more details in [8]). The spaces $\mathcal{H}_{P}$ can be identified with the space $\mathcal{F}_{\text {sym }} \otimes \mathcal{F}_{\text {sym }}=\mathcal{F}_{\text {sym }}^{\otimes 2}$ and then the operator $H_{P}$ is represented in $\mathcal{F}_{\text {sym }}^{\otimes 2}$ in the form (see [25, 3])

$$
\begin{aligned}
H_{P} & =\frac{1}{2}\left(\left(P-P_{f}\right)-e \mathcal{A}_{\varphi}(0)\right)^{2}+H_{f} \\
& =\frac{1}{2}\left(P-P_{f}\right)^{2}-e\left(P-P_{f}\right) \cdot \mathcal{A}_{\varphi}(0)+\frac{e^{2}}{2} \mathcal{A}_{\varphi}^{2}(0)+H_{f}=H_{P}^{0}+H_{\mathrm{int}},
\end{aligned}
$$

where

$$
H_{P}^{0}=\frac{1}{2}\left(P-P_{f}\right)^{2}+H_{f}
$$

Here we have used the fact that $P_{f}$ and $\mathcal{A}_{\varphi}(0)$ commute, which follows from relations (1.2a). 
We consider the following group $\mathcal{S}$ of gauge transformations of the field $\mathcal{A}(x)$ consisting of functions

$$
S: \quad R^{3} \rightarrow O(2): \quad k \rightarrow S(k) \in O(2),
$$

where $O(2)$ is the group of orthogonal $2 \times 2$ matrices (a rotation of the plane and, possibly, a reflection with respect to some straight line). Multiplication in $\mathcal{S}$ is defined by a pointwise product of matrices $S(k)$. This group transforms the creation and annihilation operators:

$$
\begin{array}{ll}
\tilde{a}_{1}(k)=s_{11}(k) a_{1}(k)+s_{12}(k) a_{2}(k), & \tilde{a}_{1}^{*}(k)=s_{11}(k) a_{1}^{*}(k)+s_{12}(k) a_{2}^{*}(k), \\
\tilde{a}_{2}(k)=s_{21}(k) a_{1}(k)+s_{22}(k) a_{2}(k), & \tilde{a}_{2}^{*}(k)=s_{21}(k) a_{1}^{*}(k)+s_{22}(k) a_{2}^{*}(k) ;
\end{array}
$$

and the vectors $e_{1}(k)$ and $e_{2}(k)$ :

$$
\begin{aligned}
& \tilde{e}_{1}(k)=s_{11}(k) e_{1}(k)+s_{12}(k) a_{2}(k), \\
& \tilde{e}_{2}(k)=s_{21}(k) a_{1}(k)+s_{22}(k) a_{2}(k),
\end{aligned}
$$

where $S(k)=\left\{s_{i_{1} i_{2}}(k)\right\}$ is an orthogonal matrix. The transformations (1.9b) are canonical transformations (that is, they preserve the commutation relations between the creation and annihilation operators) and generate some unitary transformation of the space $\mathcal{F}_{\mathrm{sym}}^{\otimes 2}$ (see [7]). Thus, for each $P$ the operator $\tilde{H}_{P}$ obtained from $H_{P}$ by replacing the operators $a_{j}(k), a_{j}^{*}(k)$ and vectors $e_{j}(k)$ by $\tilde{a}_{j}(k), \tilde{a}_{j}^{*}(k)$ and $\tilde{e}_{j}(k), j=1,2$, is unitarily equivalent to $H_{P}$.

In what follows it will be convenient for us to think of $\mathcal{F}_{\mathrm{sym}}^{\otimes 2}$ as the Hilbert space $L_{2}\left(\Gamma_{0} \times \Gamma_{0}, d \lambda\right)$ of functions defined on the set $\Gamma_{0} \times \Gamma_{0}$ of ordered pairs of finite subsets $\left(\gamma_{1}, \gamma_{2}\right), \gamma_{i} \in \Gamma_{0}, i=1,2$, of the space $R^{d}$ that are square-summable with respect to the so-called Lebesgue-Poisson measure [21]

$$
d \lambda=d \lambda\left(\gamma_{1}, \gamma_{2}\right)=\frac{\prod_{i=1}^{n_{1}} d x_{i}^{(1)} \prod_{i=1}^{n_{2}} d x_{i}^{(2)}}{n_{1} ! n_{2} !}
$$

where $\gamma_{j}=\left\{x_{i}^{(j)}\right\}_{i=1}^{n_{j}}, j=1,2$, and $n_{j}=\left|\gamma_{j}\right|$ is the cardinality of the set $\gamma_{j}, j=$ 1,2. Visually, $\gamma_{j}$ is the set of momenta of bosons that have polarization $j$. Under this realization of the Fock space $\mathcal{F}_{\text {sym }}^{\otimes 2}$, the operators introduced above are written as follows:

$$
\begin{aligned}
\left(P_{f} F\right)\left(\gamma_{1}, \gamma_{2}\right)= & \left(\sum_{k \in \gamma_{1}} k+\sum_{k \in \gamma_{2}} k\right) F\left(\gamma_{1}, \gamma_{2}\right), \\
\left(H_{f} F\right)\left(\gamma_{1}, \gamma_{2}\right)= & \left(\sum_{k \in \gamma_{1}} \omega(k)+\sum_{k \in \gamma_{2}} \omega(k)\right) F\left(\gamma_{1}, \gamma_{2}\right), \\
\left(\left(e\left(P-P_{f}\right) \cdot \mathcal{A}_{\varphi}(0)\right) F\right)\left(\gamma_{1}, \gamma_{2}\right) & \\
= & {\left[\sum_{s \in \gamma_{1}} c_{1}\left(P-\sum_{k \in \gamma_{1}} k-\sum_{k \in \gamma_{2}} k ; s\right) F\left(\gamma_{1} \backslash\{s\}, \gamma_{2}\right)\right.} \\
& +\sum_{s \in \gamma_{2}} c_{2}\left(P-\sum_{k \in \gamma_{1}} k-\sum_{k \in \gamma_{2}} k ; s\right) F\left(\gamma_{1}, \gamma_{2} \backslash\{s\}\right) \\
& +\int c_{1}\left(P-\sum_{k \in \gamma_{1}} k-\sum_{k \in \gamma_{2}} k ; q\right) F\left(\gamma_{1} \cup\{q\}, \gamma_{2}\right) d q \\
& \left.+\int c_{2}\left(P-\sum_{k \in \gamma_{1}} k-\sum_{k \in \gamma_{2}} k ; q\right) F\left(\gamma_{1}, \gamma_{2} \cup\{q\}\right) d q\right]
\end{aligned}
$$


Here,

$$
\begin{gathered}
c_{j}(r, s)=\left(r, e_{j}(s)\right) \frac{\varphi(s)}{\sqrt{2 \omega(s)}}, \quad r, s \in R^{d}, \quad j=1,2, \\
\frac{e^{2}}{2} \mathcal{A}_{\varphi}^{2}(0)=\frac{e^{2}}{2}\left[\int \int d k _ { 1 } d k _ { 2 } \left\{\sum _ { \substack { j _ { 1 } , j _ { 2 } = 1 , 2 \\
j _ { 1 } } } w _ { j _ { 1 } , j _ { 2 } } ( k _ { 1 } , k _ { 2 } ) \left(a_{j_{1}}\left(k_{1}\right) a_{j_{2}}\left(k_{2}\right)\right.\right.\right. \\
\left.\left.+2 a_{j_{1}}^{*}\left(k_{1}\right) a_{j_{2}}\left(k_{2}\right)+a_{j_{1}}^{*}\left(k_{1}\right) a_{j_{2}}^{*}\left(k_{2}\right)\right)\right\} \\
\left.+\int d k \sum_{j=1,2} w_{j, j}(k, k) \mathbb{I}\right] \\
w_{j_{1}, j_{2}}\left(k_{1}, k_{2}\right)=\frac{\varphi\left(k_{1}\right) \varphi\left(k_{2}\right)}{2 \sqrt{\omega\left(k_{1}\right) \omega\left(k_{2}\right)}}\left(e_{j_{1}}\left(k_{1}\right), e_{j_{2}}\left(k_{2}\right)\right),
\end{gathered}
$$

and II is the identity operator in $\mathcal{F}_{\text {sym }}^{\otimes 2}$.

We write out the action of each summand in (1.12).

1. The first summand:

$$
\begin{aligned}
& \left(\left(\iint\left(w_{1,1}\left(k_{1}, k_{2}\right) a_{1}\left(k_{1}\right) a_{1}\left(k_{2}\right)\right) d k_{1} d k_{2}\right) F\right)\left(\gamma_{1}, \gamma_{2}\right) \\
& \quad=\left(2 \sum_{\left\{s_{1}, s_{2}\right\} \subset \gamma_{1}} w_{1,1}\left(s_{1}, s_{2}\right) F\left(\gamma_{1} \backslash\left\{s_{1}, s_{2}\right\}, \gamma_{2}\right)\right) \cdot \theta_{2}\left(\left|\gamma_{1}\right|\right),
\end{aligned}
$$

where $\left\{s_{1}, s_{2}\right\}$ is a two-point subset, and

$$
\begin{aligned}
& \left(\left(\iint\left(w_{1,2}\left(k_{1}, k_{2}\right) a_{1}\left(k_{1}\right) a_{2}\left(k_{2}\right)\right) d k_{1} d k_{2}\right) F\right)\left(\gamma_{1}, \gamma_{2}\right) \\
& =\left(\left(\iint\left(w_{2,1}\left(k_{1}, k_{2}\right) a_{2}\left(k_{1}\right) a_{1}\left(k_{2}\right)\right) d k_{1} d k_{2}\right) F\right)\left(\gamma_{1}, \gamma_{2}\right) \\
& =\left(\sum_{\substack{s_{1} \in \gamma_{1} \\
s_{2} \in \gamma_{2}}} w_{1,2}\left(s_{1}, s_{2}\right) F\left(\gamma_{1} \backslash\left\{s_{1}\right\}, \gamma_{2} \backslash\left\{s_{2}\right\}\right)\right) \cdot \theta_{1}\left(\left|\gamma_{1}\right|\right) \cdot \theta_{1}\left(\left|\gamma_{2}\right|\right), \\
& \left(\left(\iint\left(w_{2,2}\left(k_{1}, k_{2}\right) a_{2}\left(k_{1}\right) a_{2}\left(k_{2}\right)\right) d k_{1} d k_{2}\right) F\right)\left(\gamma_{1}, \gamma_{2}\right) \\
& \left.=\left(2 \sum_{\substack{\left\{s_{1}, s_{2}\right\} \subset \gamma_{1} \\
w_{2,2}}} w_{1}, s_{2}\right) F\left(\gamma_{1}, \gamma_{2} \backslash\left\{s_{1}, s_{2}\right\}\right)\right) \cdot \theta_{2}\left(\left|\gamma_{2}\right|\right),
\end{aligned}
$$

where

$$
\theta_{a}(x)= \begin{cases}1, & x \geq a \\ 0, & x<a\end{cases}
$$


2. The second summand:

$$
\begin{array}{r}
\left.2\left(\left(\iint w_{1,1}\left(k_{1}, k_{2}\right) a_{1}^{*}\left(k_{1}\right) a_{1}\left(k_{2}\right)\right) d k_{1} d k_{2}\right) F\right)\left(\gamma_{1}, \gamma_{2}\right) \\
=2 \int \sum_{s \in \gamma_{1}} w_{1,1}(s, t) F\left(\left(\gamma_{1} \backslash\{s\}\right) \cup\{t\}, \gamma_{2}\right) d t \\
2\left(\left(\iint\left(w_{1,2}\left(k_{1}, k_{2}\right) a_{1}^{*}\left(k_{1}\right) a_{2}\left(k_{2}\right)\right) d k_{1} d k_{2}\right) F\right)\left(\gamma_{1}, \gamma_{2}\right) \\
=2 \int \sum_{s \in \gamma_{2}} w_{1,2}(t, s) F\left(\gamma_{1} \cup\{t\}, \gamma_{2} \backslash\{s\}\right) d t \\
2\left(\left(\int\left(w_{2,1}\left(k_{1}, k_{2}\right) a_{2}^{*}\left(k_{1}\right) a_{1}\left(k_{2}\right)\right) d k_{1} d k_{2}\right) F\right)\left(\gamma_{1}, \gamma_{2}\right) \\
=2 \int \sum_{s \in \gamma_{1}} w_{2,1}(s, t) F\left(\gamma_{1} \backslash\{s\}, \gamma_{2} \cup\{t\}\right) d t \\
2\left(\left(\int\left(w_{2,2}\left(k_{1}, k_{2}\right) a_{2}^{*}\left(k_{1}\right) a_{2}\left(k_{2}\right)\right) d k_{1} d k_{2}\right) F\right)\left(\gamma_{1}, \gamma_{2}\right) \\
=2 \int \sum_{s \in \gamma_{2}} w_{2,2}(s, t) F\left(\gamma_{1},\left(\gamma_{2} \backslash\{s\}\right) \cup\{t\}\right) d t .
\end{array}
$$

3. The third summand:

$$
\begin{gathered}
\left(\left(\iint\left(w_{1,1}\left(k_{1}, k_{2}\right) a_{1}^{*}\left(k_{1}\right) a_{1}^{*}\left(k_{2}\right)\right) d k_{1} d k_{2}\right) F\right)\left(\gamma_{1}, \gamma_{2}\right) \\
=\iint w_{1,1}\left(t_{1}, t_{2}\right) F\left(\gamma_{1} \cup\left\{t_{1}, t_{2}\right\}, \gamma_{2}\right) d t_{1} d t_{2} \\
\left(\left(\iint\left(w_{1,2}\left(k_{1}, k_{2}\right) a_{1}^{*}\left(k_{1}\right) a_{2}^{*}\left(k_{2}\right)\right) d k_{1} d k_{2}\right) F\right)\left(\gamma_{1}, \gamma_{2}\right) \\
\left.=\int\left(\iint\left(w_{2,1}\left(k_{1}, k_{2}\right) a_{2}^{*}\left(k_{1}\right) a_{1}^{*}\left(k_{2}\right)\right) d k_{1} d k_{2}\right) F\right)\left(\gamma_{1}, \gamma_{2}\right) \\
=\iint w_{1,2}\left(t_{1}, t_{2}\right) F\left(\gamma_{1} \cup\left\{t_{1}\right\}, \gamma_{2} \cup\left\{t_{2}\right\}\right) d t_{1} d t_{2}, \\
\left(\left(\int\left(w_{2,2}\left(k_{1}, k_{2}\right) a_{2}^{*}\left(k_{1}\right) a_{2}^{*}\left(k_{2}\right)\right) d k_{1} d k_{2}\right) F\right)\left(\gamma_{1}, \gamma_{2}\right) \\
=\iint w_{2,2}\left(t_{1}, t_{2}\right) F\left(\gamma_{1}, \gamma_{2} \cup\left\{t_{1}, t_{2}\right\}\right) d t_{1} d t_{2} .
\end{gathered}
$$

The preceding formulae give a detailed description of the action of the operator $H_{P}$. For convenience, in what follows, instead of the operator $H_{P}$ we consider the operator $\bar{H}_{P}=H_{P}-B \cdot \mathbb{I}$, where

$$
B=e^{2} \int\left(w_{1,1}(k, k)+w_{2,2}(k, k)\right) d k=\frac{e^{2}}{2} \int \frac{\varphi^{2}(k)}{\omega(k)} d k .
$$

Note that $H_{P}^{0}$ is the "free" Hamiltonian; that is, $H_{P}$ for $e=0$ has the form

$$
\left(H_{P}^{0} F\right)\left(\gamma_{1}, \gamma_{2}\right)=\left[\frac{1}{2}\left(P-\sum_{k \in \gamma_{1}} k-\sum_{k \in \gamma_{2}} k\right)^{2}+\sum_{k \in \gamma_{1}} \omega(k)+\sum_{k \in \gamma_{2}} \omega(k)\right] F\left(\gamma_{1}, \gamma_{2}\right)
$$

The operator $H_{P}^{0}$ is obviously self-adjoint and semibounded from below. 
The space $\mathcal{F}_{\mathrm{sym}}^{\otimes 2}$ decomposes in an obvious fashion into the orthogonal sum of the subspaces

$$
\mathcal{H}_{n_{1}, n_{2}}=\left\{F \in \mathcal{F}_{\text {sym }}^{2}: F\left(\gamma_{1}, \gamma_{2}\right)=0 \text { if }\left|\gamma_{1}\right| \neq n_{1} \text { or }\left|\gamma_{2}\right| \neq n_{2}\right\}
$$

which are invariant under $H_{P}^{0}$. The spectrum of $\left.H_{P}^{0}\right|_{\mathcal{H}_{n_{1} n_{2}}}$ coincides with the values of the function

$$
a_{n_{1}, n_{2}}^{(P)}\left(\gamma_{1}, \gamma_{2}\right)=\frac{1}{2}\left(P-\sum_{k \in \gamma_{1}} k-\sum_{k \in \gamma_{2}} k\right)^{2}+\sum_{k \in \gamma_{1}} \omega(k)+\sum_{k \in \gamma_{2}} \omega(k)
$$

and extends from the value $\lambda_{n_{1}, n_{2}}^{0}(P)$ to $\infty$, where

$$
\lambda_{n_{1}, n_{2}}^{0}(P)=\min _{\substack{\gamma_{1}, \gamma_{2} \\\left|\gamma_{1}\right|=n_{1},\left|\gamma_{2}\right|=n_{2}}} a_{n_{1}, n_{2}}^{(P)}\left(\gamma_{1}, \gamma_{2}\right)
$$

It is obvious that

$$
\lambda_{n_{1}, n_{2}}^{0}(P)=\lambda_{n_{1}^{\prime}, n_{2}^{\prime}}^{0}(P)
$$

for $n_{1}^{\prime}+n_{2}^{\prime}=n_{1}+n_{2}$. Therefore the actual thresholds of the spectrum $H_{P}^{0}$ can be numbered as

$$
\lambda_{0}^{(0)}(P)<\lambda_{1}^{(0)}(P)<\cdots<\lambda_{n}^{(0)}(P)<\cdots,
$$

where $\lambda_{n}^{0}(P)=\lambda_{n_{1}, n_{2}}^{(0)}(P)$ for $n_{1}+n_{2}=n$. Note that inequality (1.3) implies the inequality

$$
\lambda_{N}^{(0)}(P)>\lambda_{N^{\prime}}^{(0)}(P)+\left(N-N^{\prime}\right) c_{0}
$$

where $N>N^{\prime} \geq 1$.

In this paper we study the spectrum of the total Hamiltonian $\bar{H}_{P}$ on the ray

$$
\left(-\infty, \lambda_{2}^{(0)}(P)-\varepsilon\right),
$$

where $\varepsilon>0$ is an arbitrary fixed number. Our main result is contained in the following theorem.

\section{Theorem 1.1.}

I. Let $d \geq 3$. Then there exists $e_{0}>0$ such that for any $0<e<e_{0}$ and $P \in R^{d}$ there is a value $\lambda_{1}(P)<\lambda_{1}^{(0)}(P)$ and a bounded domain $G_{0} \subset R^{d}$ such that for $P \in G_{0}$ the operator $H_{P}$ has only one eigenvalue $\xi_{P}$ in the interval $\left(-\infty, \lambda_{1}(P)\right)$, while for $P \notin G_{0}$ there are no such eigenvalues. This eigenvalue is non-degenerate and does not exceed $\lambda_{0}^{(0)}(P)=P^{2} / 2$. Furthermore, $\lim _{P \rightarrow P^{\prime} \in \partial G_{0}} \xi_{P}=\lambda_{1}\left(P^{\prime}\right)$, where $\partial G_{0}$ is the boundary of the domain $G_{0}$. An eigenvector corresponding to the eigenvalue $\xi_{P}$ is thus a ground state of the operator $H_{P}, P \in G_{0}$. For $P \notin G_{0}$, the operators $H_{P}$ have no ground states.

II. For any pre-assigned value $0<\varepsilon<c_{0}$, there exists $\bar{e}=\bar{e}(\varepsilon) \leq e_{0}$ such that for $e<\bar{e}$ and for all $P \in R^{d}$ there exist a bounded domain $G_{1}^{\varepsilon}(P)$, a real function $\left\{\xi_{P}^{\varepsilon}(q), q \in G_{1}^{\varepsilon}(P)\right\}$ defined in this domain and taking values in the interval $\left(\lambda_{1}(P), \lambda_{2}^{0}(P)-\varepsilon\right)$, and two subspaces $\mathcal{H}_{j}^{\varepsilon}(P) \subset \mathcal{F}_{\mathrm{sym}}^{\otimes 2}, j=1,2$, invariant under $H_{P}$. The restriction of the operator $H_{P}$ to each of these subspaces is unitarily equivalent to the operator of multiplication by the function $\xi_{P}^{\varepsilon}(q)$ acting in the space $L_{2}\left(G_{1}^{\varepsilon}(P), d q\right)$. Furthermore, for $0<\varepsilon_{2}<\varepsilon_{1}$ we have $\bar{e}\left(\varepsilon_{1}\right) \geq \bar{e}\left(\varepsilon_{2}\right)$, $G_{1}^{\varepsilon_{1}}(P) \subset G_{1}^{\varepsilon_{2}}(P), \mathcal{H}_{j}^{\varepsilon_{1}}(P) \subset \mathcal{H}_{j}^{\varepsilon_{2}}(P), j=1,2$, and

$$
\left.\xi_{P}^{\varepsilon_{2}}\right|_{G_{1}^{\varepsilon_{1}}(P)}(q)=\xi_{P}^{\varepsilon_{1}}(q), q \in G_{1}^{\varepsilon_{1}}(P) .
$$




\section{Plan of the Proof of Theorem 1.1}

In this paper we follow the main methods used in 22 and [1]. For the convenience of the reader we give a brief description of our general strategy in the proof of the main theorem.

We consider the action of the resolvent $R_{\bar{H}_{P}}(z) L=F$ on an element $L \in \mathcal{F}_{\mathrm{sym}}^{\otimes 2}$. We obviously have the equation

$$
\left(\bar{H}_{P}-z \mathbb{I}\right) F=L .
$$

We represent the space $\mathcal{F}_{\mathrm{sym}}^{\otimes 2}$ in the form of the sum of spaces

$$
\mathcal{F}_{\mathrm{sym}}^{\otimes 2}=\mathcal{H}^{\leq 1} \oplus \mathcal{H}^{>1}
$$

where $\mathcal{H}^{\leq 1}=\mathcal{H}_{0,0} \oplus \mathcal{H}_{1,0} \oplus \mathcal{H}_{0,1}$ and $\mathcal{H}^{>1}=\bigoplus_{n_{1}+n_{2}>1} \mathcal{H}_{n_{1} n_{2}}$. Correspondingly, the solution $F$ of equation (2.1) and its right-hand side are represented in the form

$$
F=F_{1}+F_{2}, \quad L=L_{1}+L_{2}, \quad \text { where } \quad F_{1}, L_{1} \in \mathcal{H}^{\leq 1}, \quad F_{2}, L_{2} \in \mathcal{H}^{>1},
$$

and the operator $\bar{H}_{P}$ itself is represented by the operator matrix

$$
\bar{H}_{P} \backsim\left(\begin{array}{ll}
h_{11} & h_{12} \\
h_{21} & h_{22}
\end{array}\right),
$$

where $h_{i_{1} i_{2}}=\Pi_{i_{1}} \bar{H}_{P} \Pi_{i_{2}}, i_{1}, i_{2}=1,2$, and $\Pi_{1}$ and $\Pi_{2}$ are the projections onto the subspaces $\mathcal{H}_{P}^{\leq 1}$ and $\mathcal{H}_{P}^{>1}$, respectively. Equation (2.1) is now rewritten as the system of equations

$$
\begin{aligned}
& \left(h_{11}-z\right) F_{1}+h_{12} F_{2}=L_{1}, \\
& h_{21} F_{1}+\left(h_{22}-z\right) F_{2}=L_{2} .
\end{aligned}
$$

Lemma 2.1. Let $\bar{\lambda}_{2}(P)=\inf \operatorname{spec} h_{2,2}$. Then

$$
\bar{\lambda}_{2}(P) \leq \lambda_{2}^{0}(P)
$$

and for any $\varepsilon>0$ and sufficiently small $e$,

$$
\bar{\lambda}_{2}(P)>\lambda_{2}^{0}(P)-\varepsilon \text {. }
$$

Proof. It is obvious that

$$
\bar{\lambda}_{2}(P)=\inf _{\substack{F \in \mathcal{H}>1 \\\|F\|=1}}\left(h_{22} F, F\right) .
$$

We choose $F=\left\{f_{n_{1}, n_{2}}\right\}$ such that $f_{n_{1}, n_{2}}=0$ for $\left(n_{1}, n_{2}\right) \neq(2,0)$. Then

$$
\begin{aligned}
\left(h_{22} F, F\right)= & \frac{1}{2} \iint a_{2,0}^{P}\left(\left\{s_{1}, s_{2}\right\}, \varnothing\right) f_{2,0}^{2}\left(\left\{s_{1}, s_{2}\right\}, \varnothing\right) d s_{1} d s_{2} \\
& +e^{2} \iiint w_{1,1}(s, t) f_{2,0}(\{\bar{s}, t\}, \varnothing) f_{2,0}(\{\bar{s}, s\}, \varnothing) d s d \bar{s} d t .
\end{aligned}
$$

It is easy to verify that the minimum of the function $a_{2,0}^{P}\left(\left\{s_{1}, s_{2}\right\}, \varnothing\right)$, which is equal to $\lambda_{2}^{0}(P)$, is attained at a point $\left(s_{1}^{0}, s_{2}^{0}\right)$, where $\left(s_{1}^{0}=s_{2}^{0}=s^{0}\right)$. Let

$$
f_{2,0}\left(\left\{s_{1}, s_{2}\right\}\right)=\frac{\sqrt{2}}{|u|} \chi_{u}\left(s_{1}\right) \chi_{u}\left(s_{2}\right) \text {, }
$$

where $u \subset R^{d}$ is a neighbourhood of the point $s^{0}, \chi_{u}(\cdot)$ is the characteristic function of this neighbourhood, and $|u|$ is the volume of the neighbourhood $u$. It is easy to see that for this choice of $f_{2,0}$ and for a sufficiently small neighbourhood $u$ the quadratic form (2.6d) is equal to

$$
\lambda_{2}^{0}(P)+O(|u|)
$$


This fact and (2.6c) imply inequality (2.6a). We shall derive inequality (2.6b) later.

Now let $z$ be real and let $s<\bar{\lambda}_{2}(P)$. Then the resolvent $\left(h_{22}-z \mathbb{I}_{2}\right)^{-1}$ is defined (here $\mathbb{I}_{2}$ is the identity operator in the space $\mathcal{H}^{>1}$ ) and from the second equation (2.5) we find that $F_{2}$ is equal to

$$
F_{2}=\left(h_{22}-z \mathbb{I}_{2}\right)^{-1}\left(L_{2}-h_{21} F_{1}\right) .
$$

Substituting this expression into the first equation (2.5) we find that $F_{1}$ satisfies the equation

$$
\left(h_{11}-h_{12}\left(h_{22}-z \mathbb{I}_{2}\right)^{-1} h_{21}-z \mathbb{I}_{1}\right) F_{1}=L_{1}-h_{12}\left(h_{22}-z \mathbb{I}_{2}\right)^{-1} L_{2}
$$

(where $\mathbb{I}_{1}$ is the identity operator in $\mathcal{H}^{\leq 1}$ ).

Setting $z=\xi$ we introduce the family of operators

$$
\mathcal{A}(\xi)=h_{11}-h_{12}\left(h_{22}-\xi \mathbb{I}_{2}\right)^{-1} h_{21}, \quad-\infty<\xi<\lambda_{2}^{0}(P)-\varepsilon,
$$

acting in the space $\mathcal{H} \leq 1$.

As we shall verify below, operators in this family are generalized Friedrichs operators (see [26]); that is, they have the form

$$
\begin{aligned}
(\mathcal{A}(\xi) F)_{00} & =a_{0,0}(\xi) f_{0,0}+(\widehat{F}, v) \\
(\mathcal{A}(\xi) F)(q) & =M(q, \xi) \widehat{F}(q)+\int D\left(q, q^{\prime} ; \xi\right) \widehat{F}\left(q^{\prime}\right) d q^{\prime}+v(q) f_{00}
\end{aligned}
$$

Here,

$$
\begin{gathered}
F=\left(f_{00}, \widehat{F}(q)\right), \quad \widehat{F}(q)=\left\{f_{10}(q), f_{01}(q)\right\}, \quad v(q)=\left(v_{10}(q), v_{01}(q)\right), \\
M(q, \xi), \quad D\left(q, q^{\prime} ; \xi\right)
\end{gathered}
$$

are $2 \times 2$ matrices. Furthermore,

$$
(\widehat{F}, v)=\int\left(v_{10}(q) f_{10}(q)+v_{01}(q) f_{01}(q)\right) d q .
$$

Operators of the form (2.10) are well researched, and we use certain well-known facts relating to these operators. In particular, for each $\xi$ less than some value $\bar{\xi}=\bar{\xi}(P)$, we construct an eigenvector $\psi_{0}(\xi) \in \mathcal{H}^{\leq 1}$ with eigenvalues $E_{0}(\xi)$, as well as a system of generalized eigenvectors $\left\{\psi_{\bar{q}, j}(\xi), q \in R^{d}, j=1,2\right\}$ with eigenvalues $E_{\bar{q}, j}(\xi)$ for the operator $\mathcal{A}(\xi)$. Next, choosing $\xi=\xi_{0}$ satisfying the equation

$$
E_{0}\left(\xi_{0}\right)=\xi_{0},
$$

we obtain an eigenvector $F^{0}$ of the operator $\bar{H}_{p}$ by setting $F_{1}^{0}=\psi_{0}\left(\xi_{0}\right) \in \mathcal{H}^{\leq 1}$ and

$$
F_{2}^{0}=-\left(h_{22}-\xi_{0} \mathbb{I}_{2}\right)^{-1} h_{21} \psi_{0} \equiv S\left(\xi_{0}\right) \psi_{0} \in \mathcal{H}^{>1} .
$$

In a similar fashion we construct a system of generalized eigenvectors of $\bar{H}_{P}$ by finding a value $\xi=\xi_{\bar{q}, j}$, for each $\bar{q}$ in some domain $G_{1}^{\varepsilon}(P)$ that will be indicated below, that satisfies the equation

$$
E_{\bar{q}, j}\left(\xi_{\bar{q}, j}\right)=\xi_{\bar{q}, j}
$$

Again setting

$$
F_{1, j}^{\bar{q}}=\psi_{\bar{q}, j}\left(\xi_{\bar{q}, j}\right) \quad \text { and } \quad F_{2, j}^{\bar{q}}=-\left(h_{22}-\xi_{\bar{q}, j} \mathbb{I}_{2}\right)^{-1} h_{21} \psi_{\bar{q}, j}\left(\xi_{\bar{q}, j}\right),
$$

we obtain a generalized eigenvector $F_{j}^{\bar{q}}$ of the operator $\bar{H}_{P}$. The vector $F^{0}$ (which exists only for $P$ in some bounded domain $G_{0} \subset R^{d}$ ) is a ground state of $\bar{H}_{P}$, and the generalized eigenvectors $F_{j}^{\bar{q}}$ (which exist for $\bar{q}$ in some bounded domain $G_{1}^{\varepsilon}(P)$ ) form two invariant subspaces $\mathcal{H}_{j}, j=1,2$, of the operator $\bar{H}_{P}$. 
This is a brief description of the scheme we will follow in our constructions. We now pass to a systematic exposition of the proof of the main theorem.

3. Elimination of the higher Components of the solution of EQUation (2.1)

We write down the first equation of the system (2.5) in more detail, where

$$
\begin{gathered}
F_{1}=\left\{f_{00}, f_{10}, f_{01}\right\} \in \mathcal{H} \leq 1, \quad F_{2}=\left\{f_{n_{1}, n_{2}}, n_{1}+n_{2} \geq 2\right\} \in \mathcal{H}^{>1}, \\
f_{10}(q)=f_{10}(\{q\}, \varnothing), \quad f_{01}(q)=f_{01}(\varnothing,\{q\}), \\
L_{1}=\left\{l_{00}, l_{10}, l_{01}\right\}, \quad L_{2}=\left\{l_{n_{1}, n_{2}}, n_{1}+n_{2} \geq 2\right\}: \\
\text { 1. } \quad\left(\frac{1}{2} P^{2}-z\right) f_{00}-e \int c_{1}(P, t) f_{10}(t) d t-e \int c_{2}(P, t) f_{01}(t) d t \\
+\frac{e^{2}}{2}\left(\iint w_{1,1}\left(t_{1}, t_{2}\right) f_{20}\left(\left\{t_{1}, t_{2}\right\}, \varnothing\right) d t_{1} d t_{2}\right. \\
+\iint w_{2,2}\left(t_{1}, t_{2}\right) f_{02}\left(\varnothing,\left\{t_{1}, t_{2}\right\}\right) d t_{1} d t_{2} \\
\left.+2 \iint w_{1,2}\left(t_{1}, t_{2}\right) f_{11}\left(\left\{t_{1}\right\},\left\{t_{2}\right\}\right) d t_{1} d t_{2}\right)=l_{00} .
\end{gathered}
$$

2. $\left(\frac{1}{2}(P-q)^{2}+\omega(q)-z\right) f_{10}(q)-e c_{1}(P, q) f_{00}$

$$
\begin{aligned}
& -e \int c_{1}(P-q ; t) f_{20}(\{q, t\}, \varnothing) d t-e \int c_{2}(P-q ; t) f_{11}(\{q\},\{t\}) d t \\
& +\frac{e^{2}}{2}\left[2 \int f_{10}(t) w_{1,1}(t, q) d t+2 \int f_{01}(t) w_{2,1}(q, t) d t\right.
\end{aligned}
$$$$
+2 \iint f_{21}\left(\left\{q, t_{1}\right\}, t_{2}\right) w_{1,2}\left(t_{1}, t_{2}\right) d t_{1} d t_{2}
$$$$
+\iint f_{30}\left(\left\{q, t_{1}, t_{2}\right\}, \varnothing\right) w_{1,1}\left(t_{1}, t_{2}\right) d t_{1} d t_{2}
$$$$
\left.+\iint f_{12}\left(\{q\},\left\{t_{1}, t_{2}\right\}\right) w_{2,2}\left(t_{1}, t_{2}\right) d t_{1} d t_{2}\right]=l_{10}(q) .
$$

3. $\left(\frac{1}{2}(P-q)^{2}+\omega(q)-z\right) f_{01}(q)-e c_{2}(P, q) f_{00}$

$$
\begin{aligned}
& -e \int c_{2}(P-q ; t) f_{02}(\varnothing,\{q, t\}) d t-e \int c_{1}(P-q ; t) f_{11}(\{t\},\{q\}) d t \\
& +\frac{e^{2}}{2}\left[2 \int f_{01}(t) w_{2,2}(q, t) d t+2 \int f_{10}(t) w_{1,2}(t, q) d t\right. \\
& \quad+2 \iint f_{12}\left(t_{1},\left\{q, t_{2}\right\}\right) w_{1,2}\left(t_{1}, t_{2}\right) d t_{1} d t_{2} \\
& \quad+\iint f_{03}\left(\varnothing,\left\{q, t_{1}, t_{2}\right\}\right) w_{2,2}\left(t_{1}, t_{2}\right) d t_{1} d t_{2} \\
& \left.\quad+\iint f_{21}\left(\left\{t_{1}, t_{2}\right\}, q\right) w_{1,1}\left(t_{1}, t_{2}\right) d t_{1} d t_{2}\right]=l_{01}(q) .
\end{aligned}
$$

When $z<\bar{\lambda}_{2}(P)$, the second equation (2.5) can be rewritten in the form

$$
F_{2}+Q(z) F_{2}=\left(h_{22}^{0}-z \mathbb{I}_{2}\right)^{-1} L_{2}+G(z) F_{1},
$$


where

$$
\begin{aligned}
h_{22}^{0} & =\Pi_{2} H_{P}^{0} \Pi_{2}, \\
Q(z) & =\left(h_{22}^{0}-z \mathbb{I}_{2}\right)^{-1} H_{P, \mathrm{int}} \Pi_{2}, \\
G(z) & =-\left(h_{22}^{0}-z \mathbb{I}_{2}\right)^{-1} h_{21} .
\end{aligned}
$$

The non-zero components of the vector $G(z) F_{1}$ have the form

$$
\begin{aligned}
& \left(G(z) F_{1}\right)_{2,0}\left(\left\{k_{1}, k_{2}\right\}, \varnothing\right) \\
= & \frac{e c_{1}\left(P-k_{1}-k_{2} ; k_{1}\right) f_{10}\left(k_{2}\right)+e c_{1}\left(P-k_{1}-k_{2} ; k_{2}\right) f_{10}\left(k_{1}\right)-\frac{e^{2}}{2} w_{1,1}\left(k_{1}, k_{2}\right) f_{00}}{\frac{1}{2}\left(P-k_{1}-k_{2}\right)^{2}+\omega\left(k_{1}\right)+\omega\left(k_{2}\right)-z},
\end{aligned}
$$

$$
\begin{aligned}
& \left(G(z) F_{1}\right)_{0,2}\left(\varnothing,\left\{k_{1}, k_{2}\right\}\right) \\
= & \frac{e c_{2}\left(P-k_{1}-k_{2} ; k_{1}\right) f_{01}\left(k_{2}\right)+e c_{2}\left(P-k_{1}-k_{2} ; k_{2}\right) f_{01}\left(k_{1}\right)-\frac{e^{2}}{2} w_{2,2}\left(k_{1}, k_{2}\right) f_{00}}{\frac{1}{2}\left(P-k_{1}-k_{2}\right)^{2}+\omega\left(k_{1}\right)+\omega\left(k_{2}\right)-z},
\end{aligned}
$$

$$
\begin{aligned}
& \left(G(z) F_{1}\right)_{1,1}\left(\left\{k_{1}\right\},\left\{k_{2}\right\}\right) \\
= & \frac{e c_{2}\left(P-k_{1}-k_{2} ; k_{2}\right) f_{10}\left(k_{1}\right)+e c_{2}\left(P-k_{1}-k_{2} ; k_{1}\right) f_{01}\left(k_{2}\right)-e^{2} w_{1,2}\left(k_{1}, k_{2}\right) f_{00}}{\frac{1}{2}\left(P-k_{1}-k_{2}\right)^{2}+\omega\left(k_{1}\right)+\omega\left(k_{2}\right)-z},
\end{aligned}
$$

$$
\begin{array}{r}
(3.3 \mathrm{~d}) \quad\left(G(z) F_{1}\right)_{3,0}\left(\left\{k_{1}, k_{2}, k_{3}\right\}, \varnothing\right) \\
=-\frac{\frac{e^{2}}{2}\left(w_{1,1}\left(k_{1}, k_{2}\right) f_{10}\left(k_{3}\right)+w_{1,1}\left(k_{1}, k_{3}\right) f_{10}\left(k_{2}\right)+w_{1,1}\left(k_{2}, k_{3}\right) f_{10}\left(k_{1}\right)\right)}{\frac{1}{2}\left(P-k_{1}-k_{2}-k_{3}\right)^{2}+\omega\left(k_{1}\right)+\omega\left(k_{2}\right)+\omega\left(k_{3}\right)-z}, \\
(3.3 \mathrm{e}) \quad\left(G(z) F_{1}\right)_{0,3}\left(\varnothing,\left\{k_{1}, k_{2}, k_{3}\right\}\right) \\
=-\frac{\frac{e^{2}}{2}\left(w_{2,2}\left(k_{1}, k_{2}\right) f_{01}\left(k_{3}\right)+w_{2,2}\left(k_{3}, k_{2}\right) f_{01}\left(k_{1}\right)+w_{2,2}\left(k_{1}, k_{3}\right) f_{01}\left(k_{2}\right)\right)}{\frac{1}{2}\left(P-k_{1}-k_{2}-k_{3}\right)^{2}+\omega\left(k_{1}\right)+\omega\left(k_{2}\right)+\omega\left(k_{3}\right)-z}, \\
(3.3 \mathrm{f}) \quad\left(G(z) F_{1}\right)_{2,1}\left(\left\{k_{1}, k_{2}\right\},\{k\}\right) \\
=-\frac{\frac{e^{2}}{2}\left(w_{1,2}\left(k_{1}, k\right) f_{10}\left(k_{2}\right)+w_{1,2}\left(k_{2}, k_{1}\right) f_{10}\left(k_{1}\right)+w_{1,1}\left(k_{1}, k_{2}\right) f_{01}(k)\right)}{\frac{1}{2}\left(P-k_{1}-k_{2}-k_{3}\right)^{2}+\omega\left(k_{1}\right)+\omega\left(k_{2}\right)+\omega\left(k_{3}\right)-z}, \\
(3.3 \mathrm{~g}) \quad\left(G(z) F_{1}\right)_{1,2}\left(\{k\},\left\{k_{1}, k_{2}\right\}\right) \\
=-\frac{\frac{e^{2}}{2}\left(w_{1,2}\left(k, k_{1}\right) f_{01}\left(k_{2}\right)+w_{1,2}\left(k, k_{2}\right) f_{01}\left(k_{1}\right)+w_{2,2}\left(k, k_{2}\right) f_{10}(k)\right)}{\frac{1}{2}\left(P-k_{1}-k_{2}-k_{3}\right)^{2}+\omega\left(k_{1}\right)+\omega\left(k_{2}\right)+\omega\left(k_{3}\right)-z} .
\end{array}
$$

Lemma 3.1. For $z<\lambda_{2}^{0}(P)$ the operators $\left(h_{22}^{0}-z \mathbb{I}_{2}\right)^{-1}, G(z)$, and $Q(z)$ are bounded and their norms admit the following estimates:

$$
\begin{gathered}
\left\|\left(h_{22}^{0}-z \mathbb{I}_{2}\right)^{-1}\right\|<\frac{1}{\lambda_{2}^{0}(P)-z}, \\
\|G(z)\|<\left(\frac{B_{1}}{\lambda_{2}^{0}(P)-z}+B_{2}\right) e, \\
\|Q(z)\|<\left(\frac{B_{1}^{\prime}}{\lambda_{2}^{0}(P)-z}+B_{2}^{\prime}\right) e,
\end{gathered}
$$

where $B_{1}, B_{2}, B_{1}^{\prime}$ and $B_{2}^{\prime}$ are constants independent of $P, z$, and $e$. 
Proof. Applying inequality (1.3) repeatedly, we obtain that

$$
a_{n_{1}, n_{2}}^{(P)}\left(\gamma_{1}, \gamma_{2}\right)-z>\left(n_{1}+n_{2}-2\right) c_{0}+\lambda_{2}^{0}(P)-z, \quad n_{i}=\left|\gamma_{i}\right|, \quad i=1,2 .
$$

This immediately implies (3.4a). We will now estimate the norm $\|G(z)\|$. First we show that for $z<\lambda_{2}^{0}(P)$,

$$
\left|\frac{c_{j}\left(P-k_{1}-k_{2}, k_{i}\right)}{\frac{1}{2}\left(P-k_{1}-k_{2}\right)^{2}+\omega\left(k_{1}\right)+\omega\left(k_{2}\right)-z}\right|<\frac{5 h\left(k_{i}\right)}{\lambda_{2}^{0}(P)-z}+4 h\left(k_{i}\right), \quad j=1,2, \quad i=1,2,
$$

where $h(k)=\frac{\varphi(k)}{\sqrt{2 \omega(k)}}$. As follows from (1.11a),

$$
\left|c_{j}\left(P-k_{1}-k_{2}, k_{i}\right)\right|<\left|P-k_{1}-k_{2}\right| h\left(k_{i}\right) .
$$

Thus, in the domain

$$
\left\{k_{1}, k_{2}:\left|P-k_{1}-k_{2}\right|<5\right\}
$$

the ratio (3.5) does not exceed $\frac{5 h\left(k_{i}\right)}{\lambda_{2}^{0}(P)-z}$.

Note that the minimum point $\left(\bar{k}_{1}, \bar{k}_{2}\right)$ of the function

$$
\frac{1}{2}\left(P-k_{1}-k_{2}\right)^{2}+\omega\left(k_{1}\right)+\omega\left(k_{2}\right)
$$

is determined from the equations

$$
P-\bar{k}_{1}-\bar{k}_{2}+\frac{\bar{k}_{1}}{\sqrt{\left|\bar{k}_{1}\right|^{2}+m^{2}}}=0, \quad P-\bar{k}_{1}-\bar{k}_{2}+\frac{\bar{k}_{2}}{\sqrt{\left|\bar{k}_{2}\right|^{2}+m^{2}}}=0,
$$

which imply that $\bar{k}_{1}=\bar{k}_{2}=\bar{k}$ and

$$
\bar{k}=\frac{P}{2}+\frac{\bar{k}}{\sqrt{|\bar{k}|^{2}+m^{2}}}
$$

and, consequently,

$$
\left|\bar{k}-\frac{P}{2}\right|<\frac{1}{2}
$$

Since the absolute value of the gradient of the function $\omega(k)$ does not exceed 1 , we find that

$$
\lambda_{2}^{0}(P)=\frac{1}{2}(P-2 \bar{k})^{2}+2 \omega(\bar{k})<\frac{1}{2}+2 \omega\left(\frac{P}{2}\right)+1 .
$$

We now consider the function

$$
\frac{1}{4}\left(P-k_{1}-k_{2}\right)^{2}+\omega\left(k_{1}\right)+\omega\left(k_{2}\right) .
$$

Just as in the preceding arguments, its minimum $\bar{\lambda}_{2}(P)$ is attained at the point $(\overline{\bar{k}}, \overline{\bar{k}})$, where

$$
\overline{\bar{k}}=\frac{P}{2}+\frac{\overline{\bar{k}}}{\sqrt{\overline{\bar{k}}+m^{2}}} .
$$

Hence, $\left|\frac{P}{2}-\overline{\bar{k}}\right|<1$ and $|\bar{k}-\overline{\bar{k}}|<\frac{3}{2}$. Since

$$
\overline{\bar{\lambda}}_{2}(P)=\frac{1}{4}(P-2 \overline{\bar{k}})^{2}+2 \omega(\overline{\bar{k}}) \geq 2 \omega(\overline{\bar{k}}),
$$

we conclude that

$$
|\omega(\bar{k})-\omega(\overline{\bar{k}})|<\frac{3}{2}, \quad 4>\lambda_{2}^{0}(P)-\overline{\bar{\lambda}}_{2}(P)>0 .
$$


Since

$$
\frac{1}{4}\left(P-k_{1}-k_{2}\right)^{2}>\frac{1}{4}\left|P-k_{1}-k_{2}\right|+4
$$

for $\left|P-k_{1}-k_{2}\right|>5$, it follows that

$$
\begin{aligned}
& \frac{1}{2}(P-\left.k_{1}-k_{2}\right)^{2}+\omega\left(k_{1}\right)+\omega\left(k_{2}\right)-z \\
& \quad= \frac{1}{4}\left(P-k_{1}-k_{2}\right)^{2}+\frac{1}{4}\left(P-k_{1}-k_{2}\right)^{2}+\omega\left(k_{1}\right)+\omega\left(k_{2}\right)-z \\
& \quad>\frac{1}{4}\left|P-k_{1}-k_{2}\right|+4+\overline{\bar{\lambda}}_{2}(P)-z>\frac{1}{4}\left|P-k_{1}-k_{2}\right| .
\end{aligned}
$$

Thus, the ratio (3.5) does not exceed

$$
4 h\left(k_{i}\right)+\frac{5 h\left(k_{i}\right)}{\lambda_{2}^{0}(P)-z} .
$$

Next, it follows from (1.12a) that

$$
\left|w_{i j}\left(k_{1}, k_{2}\right)\right|<h\left(k_{1}\right) \cdot h\left(k_{2}\right)
$$

and

$$
\frac{1}{2}\left(P-k_{1}-k_{2}-k_{3}\right)^{2}+\omega\left(k_{1}\right)+\omega\left(k_{2}\right)+\omega\left(k_{3}\right)-z>\lambda_{2}^{0}(P)-z+c_{0} .
$$

Note that

$$
h(k) \in L_{2}\left(R^{d}\right)
$$

since we have assumed that the function $\varphi(k)$ decreases rapidly at $\infty$. By using formulae (3.3a) $-(3.3 \mathrm{~g})$, the estimates (3.6), (3.7), (3.8), and relation (3.8a) we obtain the estimate (3.4b).

Now we estimate the norm of $Q(z)$. The vector $\Phi$ with components

$$
\Phi\left(\gamma_{1}, \gamma_{2}\right)=\frac{\left(\left(P-P_{f}\right) \cdot \mathcal{A}_{\varphi}(0) F\right)\left(\gamma_{1}, \gamma_{2}\right)}{a_{n_{1}, n_{2}}^{P}\left(\gamma_{1}, \gamma_{2}\right)-z}, \quad n_{1}=\left|\gamma_{1}\right|, \quad n_{2}=\left|\gamma_{2}\right|,
$$

where $F \in \mathcal{H}^{>1}$, consists of four summands, $I_{1}, I_{2}, I_{3}, I_{4}$, as is evident from (1.11). Here,

$$
\left(I_{1} F\right)\left(\gamma_{1}, \gamma_{2}\right)=\frac{\sum_{s \in \gamma_{1}} c_{1}\left(P-\sum_{k \in \gamma_{1}} k-\sum_{k \in \gamma_{2}} k ; s\right) F\left(\gamma_{1} \backslash s, \gamma_{2}\right)}{a_{n_{1}, n_{2}}^{P}\left(\gamma_{1}, \gamma_{2}\right)-z} .
$$

The summand $\left(I_{2} F\right)\left(\gamma_{1}, \gamma_{2}\right)$ has a similar form with $c_{1}$ replaced by $c_{2}$ and summation over elements in $\gamma_{1}$ replaced by summation over $s \in \gamma_{2}$.

Next,

$$
\left(I_{3} F\right)\left(\gamma_{1}, \gamma_{2}\right)=\frac{\int c_{1}\left(P-\sum_{k \in \gamma_{1}} k-\sum_{k \in \gamma_{2}} k ; s\right) F\left(\gamma_{1} \cup s, \gamma_{2}\right) d s}{a_{n_{1}, n_{2}}^{P}\left(\gamma_{1}, \gamma_{2}\right)-z} .
$$

The summand $\left(I_{4} F\right)\left(\gamma_{1}, \gamma_{2}\right)$ has a similar form.

We now estimate the norm $\left\|I_{1} f\right\|$. Let $n_{1}>0, n_{1}+n_{2} \geq 2$. Then, as is evident from (1.11),

$$
\begin{aligned}
& \left\|\left(I_{1} F\right)_{n_{1}, n_{2}}\right\|_{L_{2}\left(R^{d\left(n_{1}+n_{2}\right)}\right)} \\
& <\max _{\substack{k_{1}, \ldots, k_{n_{1}} \\
\bar{k}_{1}, \ldots, \bar{k}_{n}}}\left|\frac{\left|P-\sum_{i=1}^{n_{1}} k_{i}-\sum_{i=1}^{n_{2}} \bar{k}_{i}\right|}{\frac{1}{2}\left(P-\sum_{i=1}^{n_{1}} k_{i}-\sum_{i=1}^{n_{2}} \bar{k}_{i}\right)^{2}+\sum_{i=1}^{n_{1}} \omega\left(k_{i}\right)+\sum_{i=1}^{n_{2}} \omega\left(\bar{k}_{i}\right)-z}\right| \\
& \quad \times n_{1} \cdot l\left\|F_{n_{1}-1, n_{2}}\right\|_{L_{2}\left(R^{d\left(n_{1}+n_{2}-1\right)}\right)},
\end{aligned}
$$


where $l=\|h(\cdot)\|_{L_{2}\left(R^{d}\right)}$, and

$$
\left\|F_{n_{1}, n_{2}}\right\|_{L_{2}\left(R^{\left(n_{1}+n_{2}\right) d}\right)}^{2}=\int F_{n_{1}, n_{2}}^{2}\left(\left\{k_{1}, \ldots, k_{n_{1}}\right\},\left\{\bar{k}_{1}, \ldots, \bar{k}_{n_{2}}\right\}\right) \prod d k_{i} \prod d \bar{k}_{i} .
$$

Next, by setting $t_{1}=\sum_{i=1}^{n_{1}} k_{i}, t_{2}=\sum_{i=1}^{n_{2}} \bar{k}_{i}$ and applying again inequality (1.3), we obtain

$$
\begin{aligned}
\max _{\substack{k_{1}, \ldots, k_{n_{1}} \\
\bar{k}_{1}, \ldots, \bar{k}_{n_{2}}}}\left|\frac{\left|P-\sum_{i=1}^{n_{1}} k_{i}-\sum_{i=1}^{n_{2}} \bar{k}_{i}\right|}{\frac{1}{2}\left(P-\sum_{i=1}^{n_{1}} k_{i}-\sum_{i=1}^{n_{2}} \bar{k}_{i}\right)^{2}+\sum_{i=1}^{n_{1}} \omega\left(k_{i}\right)+\sum_{i=1}^{n_{2}} \omega\left(\bar{k}_{i}\right)-z}\right| \\
\quad<\max _{t_{1}, t_{2}} \frac{\left|P-t_{1}-t_{2}\right|}{\frac{1}{2}\left(P-t_{1}-t_{2}\right)^{2}+\omega\left(t_{1}\right)+\omega\left(t_{2}\right)-z+\left(n_{1}+n_{2}-2\right) c_{0}} .
\end{aligned}
$$

By repeating the arguments used in estimating the norm $\|G(z)\|$, with some modifications, we find that for $z<\lambda_{2}^{0}(P)$ the last expression does not exceed

$$
\frac{\bar{B}}{\sqrt{n_{1}+n_{2}-2}} \text { for } n_{1}+n_{2}>2, \quad \text { and } \quad \frac{5}{\lambda_{2}^{0}(P)-z}+4 \text { for } \quad n_{1}+n_{2}=2
$$

where $\bar{B}>0$ is some constant independent of $z, P$, and $k_{1}, k_{2}$.

Thus, the norm of $I_{1} F$ does not exceed

$$
\begin{gathered}
\left(\sum_{\substack{n_{1}, n_{2} \\
n_{1}+n_{2} \geq 3 \\
n_{1}>0}} \frac{\bar{B}^{2} n_{1}^{2} l^{2}}{n_{1}+n_{2}-2} \frac{\left\|F_{n_{1}-1, n_{2}}\right\|_{L_{2}\left(R^{d\left(n_{1}+n_{2}\right)-1}\right)}^{2}}{n_{1} ! n_{2} !}+\left(\frac{5}{\lambda_{2}^{0}(P)-z}+4\right)^{2} l^{2}\left\|F_{0,1}\right\|_{L_{2}\left(R^{d}\right)}^{2}\right)^{1 / 2} \\
\leq\left(\bar{B} l \max _{n \geq 3} \frac{n}{n-2}+\frac{5 l}{\lambda_{2}^{0}-z}+4 l\right)\|F\|_{\mathcal{F}_{\text {sym }}^{2}} .
\end{gathered}
$$

The norm of the second summand $I_{2} F$ is estimated in similar fashion. We now estimate $\left\|I_{3} F\right\|$. For $n_{1}+n_{2} \geq 2$ we have

$$
\left\|\left(I_{3} F\right)_{n_{1}, n_{2}}\right\|_{L_{2}\left(R^{d\left(n_{1}+n_{2}\right)}\right)}<\frac{\bar{B} l}{\sqrt{n_{1}+n_{2}-2}}\left\|F_{n_{1}+1, n_{2}}\right\|_{L_{2}\left(R^{d\left(n_{1}+n_{2}\right)+1}\right)} .
$$

When $n_{1}+n_{2}=2$, by using again (3.5) we obtain

$$
\left\|\left(I_{3} F\right)_{n_{1}, n_{2}}\right\|_{L_{2}\left(R^{2 d}\right)}<\left(\frac{5}{\lambda_{2}^{0}(P)-z}+4\right) \cdot l\left\|F_{n_{1}+1, n_{2}}\right\|_{L_{2}\left(R^{3 d}\right)} .
$$

Thus,

$$
\begin{aligned}
\left\|I_{3} F\right\|_{\mathcal{F}_{\mathrm{sym}}^{\otimes 2}}^{2}< & \bar{B}^{2} l^{2} \sum_{\substack{n_{1}, n_{2} \\
n_{1}+n_{2} \geq 3}} \frac{n+1}{n_{1}+n_{2}-2} \frac{\left\|F_{n_{1}+1, n_{2}}\right\|_{L_{2}\left(R^{d\left(n_{1}+n_{2}\right)+1}\right)}^{2}}{\left(n_{1}+1\right) ! n_{2} !} \\
& +\left(\frac{5}{\lambda_{2}^{0}(P)-z}+4\right)^{2} \cdot l^{2} \sum_{\substack{n_{1}, n_{2} \\
n_{1}+n_{2}=2}} 3 ! \frac{\left\|F_{n_{1}+1, n_{2}}\right\|_{L_{2}\left(R^{3 d}\right)}^{2}}{\left(n_{1}+1\right) ! n_{2} !}
\end{aligned}
$$

and, consequently,

$$
\left\|I_{3} F\right\|_{\mathcal{F}_{\mathrm{sym}}^{\otimes 2}}<\left(\frac{k_{1}}{\lambda_{2}^{0}(p)-z}+k_{2}\right)\|F\|_{\mathcal{F}_{\mathrm{sym}}^{\otimes 2}}
$$


where $k_{1}, k_{2}$ are constants independent of $F, t$ and $p$. A similar estimate is obtained for $\left\|I_{4} F\right\|_{\mathcal{F}_{\mathrm{sym}}^{\otimes 2}}^{\otimes}$. From these estimates we find that

$$
\|\Phi\|_{\mathcal{F}_{\mathrm{sym}}^{\otimes 2}}<\left(\frac{B_{1}^{\prime}}{\lambda_{2}^{0}(P)-z}+B_{2}^{\prime}\right)\|F\|_{\mathcal{F}_{\mathrm{sym}}^{\otimes 2}}
$$

where the vector $\Phi$ was defined in (3.9).

Next, consider the vector $\Psi$ with components

$$
\Psi\left(\gamma_{1}, \gamma_{2}\right)=\frac{\left(\mathcal{A}_{\varphi}^{2}(0) F\right)\left(\gamma_{1}, \gamma_{2}\right)}{a_{n_{1}, n_{2}}^{(P)}\left(\gamma_{1}, \gamma_{2}\right)-z}, \quad F \in \mathcal{H}^{>1} .
$$

It is evident from (1.13) that this vector consists of three groups of summands. In each group the summands are estimated in the same way; therefore we consider only one summand from each group. Let

$$
\left(\mathcal{J}_{1} F\right)_{n_{1}, n_{2}}\left(\gamma_{1}, \gamma_{2}\right)=\frac{2 \sum_{s_{1}, s_{2} \in \gamma_{1}} w_{1,1}\left(s_{1}, s_{2}\right) F\left(\gamma_{1} \backslash\left\{s_{1}, s_{2}\right\}, \gamma_{2}\right)}{a_{n_{1}, n_{2}}^{(P)}\left(\gamma_{1}, \gamma_{2}\right)-z}, \quad n_{i}=\left|\gamma_{i}\right|, \quad i=1,2 .
$$

For $n_{1}+n_{2} \geq 4, n_{1} \geq 2$, and $z<\lambda_{2}^{0}(P)$ we have

$$
\left\|\left(\mathcal{J}_{1} F\right)_{n_{1}, n_{2}}\right\|_{L_{2}\left(R^{d\left(n_{1}+n_{2}\right)}\right)}<\frac{l^{2} n_{1}\left(n_{1}-1\right)\left\|F_{n_{1}-2, n_{2}}\right\|_{L_{2}\left(R^{d\left(n_{1}+n_{2}-2\right)}\right)}}{\lambda_{2}^{0}(P)-z+\left(n_{1}+n_{2}-2\right) c_{0}}
$$

(for $n_{1}+n_{2}<4$ the vector $\left(\mathcal{J}_{1} F\right)_{n_{1}, n_{2}}$ does not belong to $\mathcal{H}^{>1}$ ). Hence,

$$
\begin{aligned}
\left\|\left(\mathcal{J}_{1} F\right)\right\|_{\mathcal{F}_{\mathrm{sym}}^{\otimes 22}} & <\frac{l^{2}}{c_{0}}\left(\sum_{\substack{n_{1}, n_{2} \\
n_{1}+n_{2} \geq 4 \\
n_{1} \geq 2}} \frac{\left(n_{1}\left(n_{1}-1\right)\right)^{2}}{\left(n_{1}+n_{2}-2\right)^{2}} \frac{\left\|F_{n_{1}-2, n_{2}}\right\|_{L_{2}\left(R^{d\left(n_{1}+n_{2}-2\right)}\right)}}{n_{1} ! n_{2} !}\right)^{1 / 2} \\
& \leq \frac{l^{2}}{c_{0}} \max _{n \geq 4} \frac{n}{n-2}\|F\|_{\mathcal{F}_{\mathrm{sym}}^{\otimes 2}} .
\end{aligned}
$$

The other summands of the first group are estimated in a similar fashion.

Now let

$$
\begin{aligned}
&\left(\mathcal{J}_{2} F\right)_{n_{1}, n_{2}}\left(\gamma_{1}, \gamma_{2}\right)=\frac{2 \int \sum_{s \in \gamma_{1}} w_{1,1}(s, t) F\left(\left(\gamma_{1} \backslash s\right) \cup\{t\}, \gamma_{2}\right) d t}{a_{n_{1}, n_{2}}^{(P)}\left(\gamma_{1}, \gamma_{2}\right)-z} \\
& n_{i}=\left|\gamma_{i}\right|, \quad i=1,2,
\end{aligned}
$$

be a vector in the second group of summands in (3.14).

For $n_{1}+n_{2} \geq 3, n_{1} \geq 1$ we have

$$
\left\|\left(\mathcal{J}_{2} F\right)_{n_{1}, n_{2}}\right\|_{L_{2}\left(R^{d\left(n_{1}+n_{2}\right)}\right)} \leq \frac{2 \operatorname{lr} n_{1}\left\|F_{n_{1}, n_{2}}\right\|_{L_{2}\left(R^{d\left(n_{1}+n_{2}\right)}\right)}}{\lambda_{2}^{0}(P)-z+\left(n_{1}+n_{2}-2\right) c_{0}},
$$

where $r=\max _{s} h(s)$.

For $n_{1}+n_{2}=2, n_{1} \geq 1$ we have

$$
\left\|\mathcal{J}_{2} F_{n_{1}, n_{2}}\right\|_{L_{2}\left(R^{2 d}\right)} \leq \frac{2 \operatorname{lr} n_{1}\left\|F_{n_{1}, n_{2}}\right\|_{L_{2}\left(R^{2 d}\right)}}{\lambda_{2}^{0}(P)-z}
$$

Thus,

$$
\left\|\left(\mathcal{J}_{2} F\right)\right\|_{\mathcal{F}_{\mathrm{sym}}^{\otimes 2}}<2 \operatorname{lr}\left(\max _{n \geq 3} \frac{n}{n-2}+\frac{2}{\lambda_{2}^{0}(p P)-z}\right)\|F\|_{\mathcal{F}_{\mathrm{sym}}^{\otimes 2}} .
$$

A similar estimate is obtained for the other summands of the second group. 
Finally, let

$$
\left(\mathcal{J}_{3} F\right)_{n_{1}, n_{2}}\left(\gamma_{1}, \gamma_{2}\right)=\frac{2 \iint w_{1,1}\left(t_{1}, t_{2}\right) F\left(\gamma_{1} \cup\left\{t_{1}, t_{2}\right\}, \gamma_{2}\right) d t d t}{a_{n_{1}, n_{2}}^{(P)}\left(\gamma_{1}, \gamma_{2}\right)-z} .
$$

For $n_{1}+n_{2} \geq 3$ we have

$$
\left\|\left(\mathcal{J}_{3} F\right)_{n_{1}, n_{2}}\right\|_{L_{2}\left(R^{d\left(n_{1}+n_{2}\right)}\right)}<\frac{l^{2}\left\|F_{n_{1}+2, n_{2}}\right\|_{L_{2}\left(R^{d\left(n_{1}+n_{2}+2\right)}\right)}}{\left(n_{1}+n_{2}-2\right) c_{0}} .
$$

For $n_{1}+n_{2}=2$ we have

$$
\left\|\left(\mathcal{J}_{3} F\right)_{n_{1}, n_{2}}\right\|_{L_{2}\left(R^{2 d}\right)}<\frac{l^{2}\left\|F_{n_{1}+2, n_{2}}\right\|_{L\left(R^{4 d}\right)}}{\lambda_{2}^{0}(P)-z} .
$$

Hence,

$$
\left\|\left(\mathcal{J}_{3} F\right)\right\|_{\mathcal{F}_{\mathrm{Fym}}^{\otimes 2}}<l^{2}\left(\max _{n \geq 3} \frac{n+2}{n-2}+\frac{\sqrt{4 !}}{\lambda_{2}^{0}(P)-z}\right)\|F\|_{\mathcal{F}_{\mathrm{sym}}^{\otimes 2}} .
$$

The other summands of the third group are estimated in a similar fashion. From (3.16), (3.18), (3.19) we obtain the estimate

$$
\|\Psi\|_{\mathcal{F}_{\mathrm{sym}}^{\otimes 2}}<\left(\frac{K_{1}^{\prime}}{\lambda_{2}^{0}(P)-z}+K_{2}^{\prime}\right)\|F\|_{\mathcal{F}_{\mathrm{sym}}^{\otimes 2}} .
$$

Estimate (3.4c) follows from (3.13) and (3.20).

Corollary 1. For a fixed $\varepsilon>0$ there exists $\widehat{e}_{0}=\widehat{e}_{0}(\varepsilon)$ such that $\|Q(z)\|<1 / 2$ for all $e<\widehat{e}_{0}$ and $z<\lambda_{2}^{0}(P)-\varepsilon$. Hence for $e<\widehat{e}_{0}$ the resolvent

$$
\left(h_{22}-\mathbb{I}_{2} z\right)^{-1}=\left(\mathbb{I}_{2}+Q(z)\right)^{-1}\left(h_{22}^{0}-\mathbb{I}_{2} z\right)^{-1}
$$

is defined for all $z<\lambda_{2}^{0}(P)-\varepsilon$, that is, $\bar{\lambda}_{2}(P)>\lambda_{2}^{0}(P)-\varepsilon$. This proves the second assertion of Lemma 2.1.

Corollary 2. It follows from the estimates of Lemma 3.1 that the operator

$$
S(z)=\left(h_{22}-\mathbb{I}_{2} z\right)^{-1} h_{21}=\left(\mathbb{I}_{2}+Q(z)\right)^{-1}\left(h_{22}^{0}-\mathbb{I}_{2} z\right)^{-1} h_{21}=\left(\mathbb{I}_{2}+Q(z)\right)^{-1} G(z)
$$

acting from $\mathcal{H}^{\leq 1}$ into $\mathcal{H}^{>1}$ is also bounded. As established above (see (2.10a)), using this operator the higher components $F_{2}^{0}$ of the eigenvector $F^{0}$ (as well as the higher components of the generalized eigenvectors $F_{2, j}^{\bar{q}}$ ) can be reconstructed from their lower components $F_{1}^{0}$ (or $\left.F_{\bar{q}, j, 1}^{0}=\psi_{\bar{q}, j}\right)$ :

$$
\left.F_{2}^{0}=S(z) F_{1}^{0} \quad \text { for } \quad z=\xi_{0} \text { (the eigenvalue for } F^{0}\right) .
$$

We now give a more explicit expression for the action of $S(z)$ on the lower components of the eigenvector $F^{0}$. Namely, we show that the higher components $F_{2}^{0}=$ $\left\{f_{n_{1} n_{2}}\left(\gamma_{1}, \gamma_{2}\right), n_{1}+n_{2} \geq 2\right\}$ of the vector $F^{0}$ are expressed in terms of its lower components $F_{1}^{0}=\left\{f_{00}, f_{10}, f_{01}\right\}$ by the following formulae.

Lemma 3.2. The following representation for the components $\left\{f_{n_{1} n_{2}}, n_{1}+n_{2} \geq 2\right\}$ of the vector $F_{2}^{0}$ holds:

$$
\begin{aligned}
f_{n_{1} n_{2}}\left(\gamma_{1}, \gamma_{2}\right)= & \sum_{y \in \gamma_{1}} b_{10}\left(\gamma_{1}, y ; \gamma_{2}\right) f_{10}(y)+\sum_{x \in \gamma_{2}} b_{01}\left(\gamma_{1} ; \gamma_{2}, x\right) f_{01}(x) \\
& +\int_{R^{d}} d_{10}\left(\gamma_{1}, t ; \gamma_{2}\right) f_{10}(t) d t \\
& +\int_{R^{d}} d_{01}\left(\gamma_{1} ; \gamma_{2}, t\right) f_{01}(t) d t+e\left(\gamma_{1}, \gamma_{2}\right) f_{00} .
\end{aligned}
$$


Here $b_{10}\left(\gamma_{1}, y ; \gamma_{2}\right)$ is a function depending on a pair of finite subsets $\gamma_{1}, \gamma_{2} \subset R^{d}$ and a point $y \in \gamma_{1} ; \quad b_{01}\left(\gamma_{1} ; \gamma_{2}, x\right)$ is a function depending on $\gamma_{1}, \gamma_{2}$ and a point $x \in \gamma_{2}$; $d_{10}\left(\gamma_{1}, t ; \gamma_{2}\right)$ depends on $\gamma_{1}, \gamma_{2}$ and a point $t \notin \gamma_{1}$; and $d_{01}\left(\gamma_{1} ; \gamma_{2}, t\right)$ depends on $\gamma_{1}, \gamma_{2}$ and a point $t \notin \gamma_{2}$; finally, $e\left(\gamma_{1}, \gamma_{2}\right)$ is a function of $\gamma_{1}$ and $\gamma_{2}$. These functions are called coefficient functions. They are infinitely differentiable with respect to all their arguments (including the parameter $P$ ), and for any $\varepsilon>0$ for a sufficiently small e they are analytic in $z$ in the half-plane $\operatorname{Re} z<\gamma_{2}^{0}(P)-\varepsilon$. More precisely, for any $r \geq 1$ and $\varepsilon>0$ there exists a value $\widehat{e}=\widehat{e}(r, \varepsilon)$ such that for $e<\widehat{e}$ and $\operatorname{Re} z<\gamma_{2}^{0}(P)-\varepsilon$ the coefficient functions and their derivatives satisfy the estimates

$$
\begin{aligned}
& \left|\partial^{\tilde{\mathcal{A}}_{n_{1}, n_{2}}} b_{10}\left(\gamma_{1}, y ; \gamma_{2}\right)\right|<\frac{e}{\left|\lambda_{2}^{0}(P)-z\right|} C\left(\tilde{\mathcal{A}}_{n_{1}, n_{2}}\right) \prod_{q \in \gamma_{1} \backslash y} h(q) \prod_{t \in \gamma_{2}} h(t)\left(n_{1} ! n_{2} !\right)^{1 / 2}, \\
& \left|\partial^{\tilde{\mathcal{A}}_{n_{1}, n_{2}}} b_{01}\left(\gamma_{1} ; \gamma_{2}, x\right)\right|<\frac{e}{\left|\lambda_{2}^{0}(P)-z\right|} C\left(\tilde{\mathcal{A}}_{n_{1}, n_{2}}\right) \prod_{q \in \gamma_{1}} h(q) \prod_{t \in \gamma_{2} \backslash x} h(t)\left(n_{1} ! n_{2} !\right)^{1 / 2}, \\
& \left|\partial^{\tilde{\mathcal{A}}_{n_{1}, n_{2}}} d_{10}\left(\gamma_{1}, y ; \gamma_{2}\right)\right|<\frac{e}{\left|\lambda_{2}^{0}(P)-z\right|} C\left(\tilde{\mathcal{A}}_{n_{1}, n_{2}}\right) \prod_{q \in \gamma_{1} \cup\{y\}} h(q) \prod_{t \in \gamma_{2}} h(t)\left(n_{1} ! n_{2} !\right)^{1 / 2}, \\
& \left|\partial^{\tilde{\mathcal{A}}_{n_{1}, n_{2}}} d_{01}\left(\gamma_{1} ; \gamma_{2}, x\right)\right|<\frac{e}{\left|\lambda_{2}^{0}(P)-z\right|} C\left(\tilde{\mathcal{A}}_{n_{1}, n_{2}}\right) \prod_{q \in \gamma_{1}} h(q) \prod_{t \in \gamma_{2} \cup\{x\}} h(t)\left(n_{1} ! n_{2} !\right)^{1 / 2}, \\
& \left|\partial^{\mathcal{A}_{n_{1}, n_{2}}} e_{00}\left(\gamma_{1}, \gamma_{2}\right)\right|<\frac{e}{\left|\lambda_{2}^{0}(P)-z\right|} C\left(\mathcal{A}_{n_{1}, n_{2}}\right) \prod_{q \in \gamma_{1}} h(q) \prod_{t \in \gamma_{2}} h(t)\left(n_{1} ! n_{2} !\right)^{1 / 2}, \\
& \left|\frac{d}{d z} \partial^{\tilde{\mathcal{A}}_{n_{1}, n_{2}}} b_{10}\left(\gamma_{1}, y ; \gamma_{2}\right)\right|<\frac{e}{\left|\lambda_{2}^{0}(P)-z\right|^{2}} C\left(\tilde{\mathcal{A}}_{n_{1}, n_{2}}\right) \prod_{q \in \gamma_{1} \backslash y} h(q) \prod_{t \in \gamma_{2}} h(t)\left(n_{1} ! n_{2} !\right)^{1 / 2}, \\
& \left|\frac{d}{d z} \partial^{\tilde{\mathcal{A}}_{n_{1}, n_{2}}} b_{01}\left(\gamma_{1} ; \gamma_{2}, x\right)\right|<\frac{e}{\left|\lambda_{2}^{0}(P)-z\right|^{2}} C\left(\tilde{\mathcal{A}}_{n_{1}, n_{2}}\right) \prod_{q \in \gamma_{1}} h(q) \prod_{t \in \gamma_{2} \backslash x} h(t)\left(n_{1} ! n_{2} !\right)^{1 / 2}, \\
& \left|\frac{d}{d z} \partial^{\tilde{\mathcal{A}}_{n_{1}, n_{2}}} d_{10}\left(\gamma_{1}, y ; \gamma_{2}\right)\right|<\frac{e}{\left|\lambda_{2}^{0}(P)-z\right|^{2}} C\left(\tilde{\mathcal{A}}_{n_{1}, n_{2}}\right) \prod_{q \in \gamma_{1} \cup\{y\}} h(q) \prod_{t \in \gamma_{2}} h(t)\left(n_{1} ! n_{2} !\right)^{1 / 2}, \\
& \left|\frac{d}{d z} \partial^{\tilde{\mathcal{A}}_{n_{1}, n_{2}}} d_{01}\left(\gamma_{1} ; \gamma_{2}, x\right)\right|<\frac{e}{\left|\lambda_{2}^{0}(P)-z\right|^{2}} C\left(\tilde{\mathcal{A}}_{n_{1}, n_{2}}\right) \prod_{q \in \gamma_{1}} h(q) \prod_{t \in \gamma_{2} \cup\{x\}} h(t)\left(n_{1} ! n_{2} !\right)^{1 / 2}, \\
& \left|\frac{d}{d z} \partial^{\mathcal{A}_{n_{1}, n_{2}}} e_{00}\left(\gamma_{1}, \gamma_{2}\right)\right|<\frac{e}{\left|\lambda_{2}^{0}(P)-z\right|^{2}} C\left(\mathcal{A}_{n_{1}, n_{2}}\right) \prod_{q \in \gamma_{1}} h(q) \prod_{t \in \gamma_{2}} h(t)\left(n_{1} ! n_{2} !\right)^{1 / 2} .
\end{aligned}
$$

Here

$$
\mathcal{A}_{n_{1}, n_{2}}=\left\{\alpha_{1}, \ldots, \alpha_{n_{1}}, \widehat{\alpha}_{1}, \ldots, \widehat{\alpha}_{n_{2}} ; \beta\right\} \quad \text { and } \quad \tilde{\mathcal{A}}_{n_{1}, n_{2}}=\left\{\alpha_{1}, \ldots, \alpha_{n_{1}}, \widehat{\alpha}_{1}, \ldots, \widehat{\alpha}_{n_{2}} ; \beta ; \delta\right\}
$$

are multi-indices, where

$$
\begin{aligned}
\alpha_{i}=\left(\alpha_{i}^{1}, \ldots, \alpha_{i}^{d}\right), & \quad \widehat{\alpha}_{j}=\left(\widehat{\alpha}_{j}^{1}, \ldots, \widehat{\alpha}_{j}^{d}\right), \quad i=1, \ldots, n_{1}, j=1, \ldots, n_{2}, \\
\beta & =\left(\beta^{1}, \ldots, \beta^{d}\right), \quad \delta=\left(\delta^{1}, \ldots, \delta^{d}\right), \\
\partial^{\mathcal{A}_{n_{1}, n_{2}}} & =\prod_{q_{i} \in \gamma_{1}} \partial_{q_{i}}^{\alpha_{i}} \prod_{t_{j} \in \gamma_{2}} \partial_{t_{j}}^{\widehat{\alpha}_{i}} \partial_{P}^{\beta}, \\
\partial^{\tilde{\mathcal{A}}_{n_{1}, n_{2}}} & =\prod_{q_{i} \in \gamma_{1}} \partial_{q_{i}}^{\alpha_{i}} \prod_{t_{j} \in \gamma_{2}} \partial_{t_{j}}^{\widehat{\alpha}_{i}} \partial_{P}^{\beta} \partial_{y}^{\delta} \quad \text { in the case of } d_{10} \text { and } b_{10}, \text { or } \\
\partial^{\tilde{\mathcal{A}}_{n_{1}, n_{2}}} & =\prod_{q_{i} \in \gamma_{1}} \partial_{q_{i}}^{\alpha_{i}} \prod_{t_{j} \in \gamma_{2}} \partial_{t_{j}}^{\widehat{\alpha}_{i}} \partial_{P}^{\beta} \partial_{x}^{\delta} \quad \text { in the case of } d_{01} \text { and } b_{01} .
\end{aligned}
$$


Furthermore,

$\left|\mathcal{A}_{n_{1}, n_{2}}\right|=\sum_{i=1}^{n_{1}}\left|\alpha_{i}\right|+\sum_{j=1}^{n_{2}}\left|\widehat{\alpha}_{j}\right|+|\beta| \leq r \quad$ and $\quad\left|\tilde{\mathcal{A}}_{n_{1}, n_{2}}\right|=\sum_{i=1}^{n_{1}}\left|\alpha_{i}\right|+\sum_{j=1}^{n_{2}}\left|\widehat{\alpha}_{j}\right|+|\beta|+|\delta| \leq r$.

The constants $C\left(A_{n_{1}, n_{2}}\right)$ and $C\left(\tilde{\mathcal{A}}_{n_{1}, n_{2}}\right)$ above depend only on these multi-indices.

The proof of Lemma 3.2 is given in the Appendix.

It follows from this lemma that the components $f_{2,0}, f_{0,2}, f_{1,1}, f_{2,1}, f_{1,2}, f_{3,0}, f_{0,3}$ of the vector $F_{2}$ occurring in the first group of equations, that is, in the system (3.1a), can be represented in the form

$$
\begin{aligned}
& f_{2,0}\left(\left\{k_{1}, k_{2}\right\} ; \varnothing\right)=b_{10}\left(\left\{k_{1}, k_{2}\right\}, k_{1} ; \varnothing\right) f_{10}\left(k_{1}\right)+b_{10}\left(\left\{k_{1}, k_{2}\right\}, k_{2} ; \varnothing\right) f_{10}\left(k_{2}\right) \\
& +\int d_{10}\left(\left\{k_{1}, k_{2}\right\}, t ; \varnothing\right) f_{10}(t) d t \\
& +\int d_{01}\left(\left\{k_{1}, k_{2}\right\} ; \varnothing, t\right) f_{01}(t) d t+e\left(\left\{k_{1}, k_{2}\right\} ; \varnothing\right) f_{0,0} ; \\
& f_{0,2}\left(\varnothing ;\left\{k_{1}, k_{2}\right\}\right)=b_{01}\left(\varnothing ;\left\{k_{1}, k_{2}\right\}, k_{1}\right) f_{01}\left(k_{1}\right)+b_{01}\left(\varnothing ;\left\{k_{1}, k_{2}\right\}, k_{2}\right) f_{01}\left(k_{2}\right) \\
& +\int d_{01}\left(\varnothing ;\left\{k_{1}, k_{2}\right\}, t\right) f_{01}(t) d t \\
& +\int d_{10}\left(\varnothing, t ;\left\{k_{1}, k_{2}\right\}\right) f_{10}(t) d t+e\left(\varnothing ;\left\{k_{1}, k_{2}\right\}\right) f_{0,0} ; \\
& f_{1,1}\left(\left\{k_{1}\right\},\left\{k_{2}\right\}\right)=b_{10}\left(\left\{k_{1}\right\}, k_{1} ;\left\{k_{2}\right\}\right) f_{10}\left(k_{1}\right)+b_{01}\left(\left\{k_{1}\right\} ;\left\{k_{2}\right\}, k_{2}\right) f_{01}\left(k_{2}\right) \\
& +\int d_{10}\left(\left\{k_{1}\right\}, t ;\left\{k_{2}\right\}\right) f_{10}(t) d t \\
& +\int d_{01}\left(\left\{k_{1}\right\} ;\left\{k_{2}\right\}, t\right) f_{01}(t) d t+e_{1,1}\left(\left\{k_{1}\right\} ;\left\{k_{2}\right\}\right) f_{0,0} ; \\
& f_{2,1}\left(\left\{k_{1}, k_{2}\right\} ;\{k\}\right)=b_{10}\left(\left\{k_{1}, k_{2}\right\}, k_{1} ;\{k\}\right) f_{10}\left(k_{1}\right)+b_{10}\left(\left\{k_{1}, k_{2}\right\}, k_{2} ;\{k\}\right) f_{10}\left(k_{2}\right) \\
& +b_{01}\left(\left\{k_{1}, k_{2}\right\} ;\{k\}, k\right) f_{01}(k)+\int d_{10}\left(\left\{k_{1}, k_{2}\right\} ; t ;\{k\}\right) f_{10}(t) d t \\
& +\int d_{01}\left(\left\{k_{1}, k_{2}\right\} ;\{k\}, t\right) f_{01}(t) d t+e_{2,1}\left(\left\{k_{1}, k_{2}\right\} ;\{k\}\right) f_{0,0} ; \\
& f_{1,2}\left(\{k\} ;\left\{k_{1}, k_{2}\right\}\right)=b_{10}\left(\{k\}, k ;\left\{k_{1}, k_{2}\right\}\right) f_{10}(k)+b_{01}\left(\{k\} ;\left\{k_{1}, k_{2}\right\}, k_{1}\right) f_{01}\left(k_{1}\right) \\
& +b_{01}\left(\{k\} ;\left\{k_{1}, k_{2}\right\}, k_{2}\right) f_{01}\left(k_{2}\right)+\int d_{10}\left(\{k\}, t ;\left\{k_{1}, k_{2}\right\}\right) f_{10}(t) d t \\
& +\int d_{01}\left(\{k\} ;\left\{k_{1}, k_{2}\right\}, t\right) f_{01}(t) d t ; \\
& f_{3,0}\left(\left\{k_{1}, k_{2}, k_{3}\right\} ; \varnothing\right)=b_{10}\left(\left\{k_{1}, k_{2}, k_{3}\right\}, k_{1} ; \varnothing\right) f_{10}\left(k_{1}\right)+b_{10}\left(\left\{k_{1}, k_{2}, k_{3}\right\}, k_{2} ; \varnothing\right) f_{10}\left(k_{2}\right) \\
& +b_{10}\left(\left\{k_{1}, k_{2}, k_{3}\right\}, k_{3} ; \varnothing\right) f_{10}\left(k_{3}\right)+\int d_{10}\left(\left\{k_{1}, k_{2}, k_{3}\right\}, t ; \varnothing\right) f_{10}(t) d t \\
& +\int d_{01}\left(\left\{k_{1}, k_{2}, k_{3}\right\}, \varnothing, t\right) f_{01}(t) d t+e_{3,0}\left(\left\{k_{1}, k_{2}, k_{3}\right\} ; \varnothing\right) f_{0,0} ; \\
& f_{0,3}\left(\varnothing,\left\{k_{1}, k_{2}, k_{3}\right\}\right)=b_{01}\left(\varnothing ;\left\{k_{1}, k_{2}, k_{3}\right\}, k_{1}\right) f_{01}\left(k_{1}\right)+b_{01}\left(\varnothing ;\left\{k_{1}, k_{2}, k_{3}\right\}, k_{2}\right) f_{01}\left(k_{2}\right) \\
& +b_{01}\left(\varnothing ;\left\{k_{1}, k_{2}, k_{3}\right\}, k_{3}\right) f_{01}\left(k_{3}\right)+\int d_{10}\left(\varnothing, t ;\left\{k_{1}, k_{2}, k_{3}\right\}\right) f_{10}(t) d t \\
& +\int d_{01}\left(\varnothing ;\left\{k_{1}, k_{2}, k_{3}\right\}, t\right) f_{01}(t) d t
\end{aligned}
$$


Substituting this expression into the system of equations (3.1a) with $l_{00}=l_{10}=l_{01}=0$ and setting $z=\xi$ we now obtain the equation

$$
\mathcal{A}(\xi) F_{1}-\xi F_{1}=0
$$

where the operator $\mathcal{A}(\xi)$ takes the form (2.10) with constant

$$
a_{00}(\xi)=a_{00}(P, \xi)
$$

given by

$$
\begin{aligned}
a_{00}(P, \xi)=\frac{P^{2}}{2}+\frac{e^{2}}{2}\left[\iint(\right. & e_{2,0}\left(\left\{t_{1}, t_{2}\right\} ; \varnothing\right) w_{1,1}\left(t_{1}, t_{2}\right) \\
& +e_{0,2}\left(\varnothing ;\left\{t_{1}, t_{2}\right\}\right) w_{2,2}\left(t_{1}, t_{2}\right) \\
& \left.\left.+2 e_{1}\left(\left\{t_{1}\right\} ;\left\{t_{2}\right\}\right) w_{1,2}\left\{t_{1}, t_{2}\right\}\right) d t_{1} d t_{2}\right]
\end{aligned}
$$

and functions

$$
v_{1,0}(t ; \xi)=v_{1,0}(t) \quad \text { and } \quad v_{0,1}(t ; \xi)=v_{0,1}(t)
$$

where

$$
\begin{aligned}
v_{1,0}(t)=-e c_{1}(P ; t)+e^{2}\left[\int b_{10}(\{s, t\}, t ; \varnothing) w_{1,1}(s, t) d s\right. \\
+\int b_{10}(\{t\}, t ;\{s\}) w_{1,2}(t, s) d s \\
+\frac{1}{2} \iint d_{10}\left(\left\{t_{1}, t_{2}\right\}, t ; \varnothing\right) w_{1,1}\left(t_{1}, t_{2}\right) d t_{1} d t_{2} \\
+\frac{1}{2} \iint d_{10}\left(\varnothing, t ;\left\{t_{1}, t_{2}\right\}\right) w_{2,2}\left(t_{1}, t_{2}\right) d t_{1} d t_{2} \\
\left.+\iint d_{10}\left(\left\{t_{1}\right\}, t ;\left\{t_{2}\right\}\right) w_{1,2}\left\{t_{1}, t_{2}\right\} d t_{1} d t_{2}\right], \\
v_{0,1}(t)=-e c_{2}(P ; t)+e^{2}\left[\int b_{01}(\varnothing,\{t, s\} ; t) w_{2,2}(s, t) d s\right. \\
+\int b_{01}(\{s\} ;\{t\}, t) w_{1,2}(s, t) d s \\
+\frac{1}{2} \iint d_{01}\left(\varnothing ;\left\{t_{1}, t_{2}\right\}, t\right) w_{2,2}\left(t_{1}, t_{2}\right) d t_{1} d t_{2} \\
+\frac{1}{2} \iint d_{01}\left(\left\{t_{1}, t_{2}\right\} ;\{\varnothing\}, t\right) w_{1,1}\left(t_{1}, t_{2}\right) d t_{1} d t_{2} \\
\left.+\iint d_{01}\left(\left\{t_{1}\right\} ;\left\{t_{2}\right\}, t\right) w_{1,2}\left\{t_{1}, t_{2}\right\} d t_{1} d t_{2}\right],
\end{aligned}
$$


with the matrix $M(q, \xi)$ given by

$$
M(q, \xi)=\left(\begin{array}{cc}
\frac{1}{2}(P-q)^{2}+\omega(q)+m_{1,1}(q ; \xi) & 0 \\
0 & \frac{1}{2}(P-q)^{2}+\omega(q)+m_{2,2}(q ; \xi)
\end{array}\right)
$$

where

$$
\begin{aligned}
m_{1,1}(q ; \xi)= & -e \int c_{1}(P-q ; t) b_{10}(\{t, q\}, q ;\{\varnothing\}) d t \\
& -e \int c_{2}(P-q ; t) b_{10}(\{q\}, q ;\{t\}) d t \\
& +\frac{e^{2}}{2} \iint w_{1,1}\left(t_{1}, t_{2}\right) b_{10}\left(\left\{t_{1}, t_{2}, q\right\}, q ; \varnothing\right) d t_{1} d t_{2} \\
& +\frac{e^{2}}{2} \iint w_{2,2}\left(t_{1}, t_{2}\right) b_{10}\left(\{q\}, q ;\left\{t_{1}, t_{2}\right\}\right) d t_{1} d t_{2} \\
& +e^{2} \iint w_{1,2}\left(t_{1}, t_{2}\right) b_{10}\left(\{t, q\}, q ;\left\{t_{2}\right\}\right) d t_{1} d t_{2} ;
\end{aligned}
$$

$$
\begin{aligned}
m_{2,2}(q ; \xi)= & -e \int c_{2}(P-q ; t) b_{01}(\varnothing ;\{t, q\}, q) d t \\
& -e \int c_{1}(P-q ; t) b_{01}(\{t\} ;\{q\}, q) d t \\
& +\frac{e^{2}}{2} \iint w_{2,2}\left(t_{1}, t_{2}\right) b_{01}\left(\varnothing ;\left\{t_{1}, t_{2}, q\right\}, q\right) d t_{1} d t_{2} \\
& +\frac{e^{2}}{2} \iint w_{1,1}\left(t_{1}, t_{2}\right) b_{01}\left(\left\{t_{1}, t_{2}\right\} ;\{q\}, q\right) d t_{1} d t_{2} \\
& +e^{2} \iint w_{1,2}\left(t_{1}, t_{2}\right) b_{01}\left(\left\{t_{1}\right\} ;\left\{t_{2}, q\right\}, q\right) d t_{1} d t_{2}
\end{aligned}
$$

and, finally, with the matrix

$$
D\left(q, q^{\prime} ; \xi\right)=\left(\begin{array}{cc}
D_{1,1}\left(q, q^{\prime} ; \xi\right) & D_{1,2}\left(q, q^{\prime} ; \xi\right) \\
D_{2,1}\left(q, q^{\prime} ; \xi\right) & D_{2,2}\left(q, q^{\prime} ; \xi\right)
\end{array}\right)
$$


THE HAMILTONIAN OF THE SPINLESS PAULI-FIERZ MODEL

where

$$
\begin{aligned}
& D_{1,1}\left(q, q^{\prime} ; \xi\right)=-e c_{1}\left(P-q, q^{\prime}\right) b_{10}\left(\left\{q, q^{\prime}\right\}, q^{\prime} ;\{s\}\right) \\
& -e \int c_{1}(P-q ; s) d_{10}\left(\left\{q, q^{\prime}, s\right\}, q^{\prime} ;\{\varnothing\}\right) d s \\
& -e \int c_{2}(P-q ; s) d_{10}\left(\left\{q, q^{\prime}\right\}, q^{\prime} ;\{s\}\right) d s \\
& +\frac{e^{2}}{2} w_{1,1}\left(q, q^{\prime}\right)+e^{2} \int w_{1,2}\left(q^{\prime}, s\right) b_{10}\left(\left\{q, q^{\prime}\right\}, q^{\prime} ;\{\varnothing\}\right) d s \\
& +e^{2} \iint w_{1,2}\left(s_{1}, s_{2}\right) d_{10}\left(\left\{q, q^{\prime}, s_{1}\right\}, q^{\prime} ;\left\{s_{2}\right\}\right) d s_{1} d s_{2} \\
& +e^{2} \int w_{1,1}\left(s, q^{\prime}\right) b_{10}\left(\left\{q, q^{\prime}, s\right\}, q^{\prime} ;\{\varnothing\}\right) d s \\
& +\frac{e^{2}}{2} \iint w_{1,1}\left(s_{1}, s_{2}\right) d_{10}\left(\left\{s_{1}, s_{2}, q\right\}, q^{\prime} ;\{\varnothing\}\right) d s_{1} d s_{2} \\
& +\frac{e^{2}}{2} \iint w_{2,2}\left(s_{1}, s_{2}\right) d_{10}\left(\{q\}, q^{\prime} ;\left\{s_{1}, s_{2}\right\}\right) d s_{1} d s_{2} ; \\
& D_{1,2}\left(q, q^{\prime} ; \xi\right)=-e \int c_{1}(P-q ; s) d_{01}\left(\{q, s\} ;\{\varnothing\}, q^{\prime}\right) d s \\
& -e c_{2}\left(P-q ; q^{\prime}\right) b_{01}\left(\{q\} ;\left\{q^{\prime}\right\}, q^{\prime}\right) \\
& -e \int c_{2}(P-q ; s) d_{01}\left(\{q\} ;\{s\}, q^{\prime}\right) d s \\
& +e^{2} w_{2,1}\left(q, q^{\prime}\right)+e^{2} \int w_{1,2}\left(s, q^{\prime}\right) b_{01}\left(\{q, s\} ;\{\varnothing\}, q^{\prime}\right) d s \\
& +e^{2} \iint w_{1,2}\left(s_{1}, s_{2}\right) d_{01}\left(\left\{q, s_{1}\right\} ;\left\{s_{2}\right\}, q^{\prime}\right) d s_{1} d s_{2} \\
& +\frac{e^{2}}{2} \iint w_{1,1}\left(s_{1}, s_{2}\right) d_{01}\left(\left\{s_{1}, s_{2}, q\right\} ;\{\varnothing\}, q^{\prime}\right) d s_{1} d s_{2} \\
& +\frac{e^{2}}{2} \iint w_{1,1}\left(s_{1}, s_{2}\right) d_{01}\left(\{q\} ;\left\{s_{1}, s_{2}\right\}, q^{\prime}\right) d s_{1} d s_{2} \\
& +e^{2} \int w_{2,2}\left(s, q^{\prime}\right) b_{01}\left(\{q\} ;\left\{q^{\prime}, s\right\}, s\right) d s ; \\
& D_{2,2}\left(q, q^{\prime} ; \xi\right)=-e c_{2}\left(P-q ; q^{\prime}\right) b_{01}\left(\{\varnothing\} ;\left\{q, q^{\prime}\right\}, q^{\prime}\right) \\
& -e \int c_{2}(P-q ; s) d_{01}\left(\{\varnothing\} ;\{s, q\}, q^{\prime}\right) d s \\
& -e \int c_{1}(P-q ; s) d_{01}\left(\{s\} ;\{q\}, q^{\prime}\right) d s \\
& +\frac{e^{2}}{2} w_{2,2}\left(q, q^{\prime}\right)+e^{2} \int w_{2,1}\left(s, q^{\prime}\right) b_{01}\left(\{s\} ;\left\{q, q^{\prime}\right\}, q^{\prime}\right) d s \\
& +\frac{e^{2}}{2} \iint w_{1,1}\left(s_{1}, s_{2}\right) d_{01}\left(\left\{s_{1}, s_{2}\right\} ;\{q\}, q^{\prime}\right) d s_{1} d s_{2} \\
& +e^{2} \iint w_{2,1}\left(s_{1}, s_{2}\right) d_{01}\left(\left\{s_{1}\right\} ;\left\{s_{2}, q\right\}, q^{\prime}\right) d s_{1} d s_{2} \\
& +e^{2} \int w_{2,2}\left(q^{\prime}, s\right) b_{01}\left(\{\varnothing\} ;\left\{q, q^{\prime}, s\right\}, q^{\prime}\right) d s \\
& +\frac{e^{2}}{2} \iint w_{2,2}\left(s_{1}, s_{2}\right) d_{01}\left(\{\varnothing\} ;\left\{s_{1}, s_{2}, q\right\}, q^{\prime}\right) d s_{1} d s_{2} .
\end{aligned}
$$


Since the operator $\mathcal{A}(\xi)$ is self-adjoint (for real $\xi$ ), the kernel satisfies

$$
D_{2,1}\left(q, q^{\prime}, \xi\right)=\overline{D_{1,2}\left(q^{\prime}, q, \xi\right)} .
$$

Remark 1 . As we mentioned in the Introduction, the action (1.9b) of the gauge transformations $S=\left\{S(k), k \in R^{d}\right\}$ generates a unitary map $T_{S}$ of the space $\mathcal{F}_{\mathrm{sym}}^{\otimes 2}$ into itself. It is easy to verify that each of the spaces

$$
\mathcal{H}^{N}=\bigoplus_{n_{1}+n_{2}=N} \mathcal{H}_{n_{1}, n_{2}}
$$

is invariant under $T_{S}$. In particular, in the space $\mathcal{H}^{0}$ (vacuum) the map $T_{S}$ acts as the identity, and in the space $\mathcal{H}_{1}=\mathcal{H}_{1,0} \oplus \mathcal{H}_{0,1}$ consisting of the pair of functions $\left\{f_{10}(q), f_{01}(q)\right\}$ it transforms these functions according to the formulae

$$
\begin{aligned}
& \tilde{f}_{10}=s_{11}(q) f_{10}(q)+s_{12}(q) f_{01}(q), \\
& \tilde{f}_{01}=s_{21}(q) f_{10}(q)+s_{22}(q) f_{01}(q),
\end{aligned}
$$

where $\left\{s_{i j}(q)\right\}_{i, j=1,2}$ is the matrix of the gauge transformation $S(q)$. Furthermore, the matrix $M(q, \xi)$ is transformed by the rule

$$
M(q, \xi) \rightarrow S^{-1}(q) M(q, \xi) S(q)
$$

and remains a diagonal matrix for any $S(q)$. Hence the matrix $M(q, \xi)$ is a multiple of the identity matrix, that is,

$$
m_{11}(q ; \xi)=m_{22}(q ; \xi)=m(q ; \xi) .
$$

Remark 2. Expanding with respect to the parameter $e$, the function $m(q ; \xi)$ has leading term

$$
m(q ; \xi)=-e^{2} \int \frac{c_{1}^{2}(P-q ; t)+c_{2}^{2}(P-q ; t)}{\frac{1}{2}(P-q-t)^{2}+\omega(q)+\omega(t)-\xi} d t+O\left(e^{3}\right) .
$$

From this it is evident that $m(q ; \xi)<0$ for small $e$, and for fixed $q$ the function $m(q ; \xi)$ decreases monotonically as $\xi$ increases.

Remark 3. For sufficiently small $e$ and for all $\xi<\lambda_{2}^{0}(P)-\varepsilon$, the function

$$
a_{P, 1}(q, \xi) \equiv \frac{1}{2}(P-q)^{2}+\omega(q)+m(q ; \xi)
$$

has a unique minimum point $q_{0}=q_{0}(\xi)$; this minimum is non-degenerate and its value is

$$
\tilde{\lambda}_{1}(P, \xi)=a_{P, 1}\left(q_{0}, \xi\right)<\lambda_{1}^{0}(P) .
$$

Furthermore, the function $a_{P, 1}(q, \xi)$ satisfies the following conditions:

$$
\begin{aligned}
c_{2}<\left|a_{P, 1}(q, \xi)\right| & <c_{0}|q|^{2}+c_{1}, \\
\left|\nabla_{q} a_{P, 1}(q, \xi)\right| & <c_{0}(|q|+1), \\
\left|\partial_{q}^{\alpha} a_{P, 1}(q, \xi)\right| & <c_{0} \quad \text { for }|\alpha| \geq 2,
\end{aligned}
$$

where $c_{0}, c_{1}, c_{2}$ are positive constants.

Remark 4 . The functions $v_{1,0}(t)$ and $v_{0,1}(t)$ are smooth functions and satisfy the estimate

$$
\left|v_{\alpha}(t)\right|<e|p| h(t), \quad \alpha=(1,0),(0,1) .
$$

Taking the leading term in $e$, these functions are equal to

$$
v_{1,0}(t)=e c_{1}(P, t)+o\left(e^{2}\right), \quad v_{0,1}(t)=e c_{2}(P, t)+o\left(e^{2}\right) .
$$


Remark 5. The matrix $\left\{D_{i, j}\left(q, q^{\prime} ; \xi\right)\right\}_{i, j=1,2}$ is a smooth function of the variables $q, q^{\prime}$ and the following estimates hold for the derivatives of its matrix elements:

$$
\max _{\substack{i, j \\|\alpha| \leq 3,\left|\alpha^{\prime}\right| \leq 3}}\left|\partial_{q}^{\alpha} \partial_{q^{\prime}}^{\alpha^{\prime}} D_{i, j}\left(q, q^{\prime} ; \xi\right)\right|<N h(q) h\left(q^{\prime}\right) .
$$

Here $\alpha=\left(\alpha^{1}, \alpha^{2}, \alpha^{3}\right)$ and $\alpha^{\prime}=\left(\alpha^{1}, \alpha^{\prime 2}, \alpha^{\prime 3}\right)$ are multi-indices, where $|\alpha|=\max \alpha^{i}$, and $\left|\alpha^{\prime}\right|=\max \alpha^{\prime i}$. The linear space of matrix kernels satisfying condition (3.35) is denoted by $\mathcal{B}$. In $\mathcal{B}$ we introduce the norm

$$
\|D\|=\inf N
$$

where the infimum is taken over all the numbers $N$ for which the estimate (3.35) holds.

Taking the leading term in $e$, the matrix elements of the matrix $D$ are equal to

$$
D_{i_{1}, i_{2}}\left(q, q^{\prime} ; \xi\right)=e^{2}\left(\frac{c_{i_{1}}\left(P-q^{\prime} ; q\right) c_{i_{2}}\left(P-q^{\prime} ; q\right)}{\frac{1}{2}\left(P-q-q^{\prime}\right)^{2}+\omega(q)+\omega\left(q^{\prime}\right)-\xi}+w_{i_{1}, i_{2}}\left(q, q^{\prime}\right)\right)+O\left(e^{3}\right) .
$$

Remark 6. In terms of $e$, the leading term of the second summand in the expression (3.27) for the function $a_{0,0}(P, \xi)$ is equal to

$$
R(\xi, P)=-\frac{e^{4}}{2} \iint \frac{w_{1,1}^{2}\left(t_{1}, t_{2}\right)+w_{2,2}^{2}\left(t_{1}, t_{2}\right)+2 w_{1,2}^{2}\left(t_{1}, t_{2}\right)}{\frac{1}{2}\left(P-t_{1}-t_{2}\right)^{2}+\omega\left(t_{1}\right)+\omega\left(t_{2}\right)-\xi} d t_{1} d t_{2}+O\left(e^{5}\right) .
$$

\section{Analysing the operator $\mathcal{A}(\xi)$}

4.1. Calculating the resolvent $(\mathcal{A}(\xi)-w I)^{-1}$. First we consider an auxiliary operator $B(\xi)$ acting in the space $\mathcal{H}_{1}=\mathcal{H}_{1,0} \oplus \mathcal{H}_{0,1}$ by the formula

$$
(B(\xi) \widehat{F})(q)=M(q ; \xi) \widehat{F}(q)+\int D\left(q, q^{\prime} ; \xi\right) \widehat{F}\left(q^{\prime}\right) d q^{\prime},
$$

where $M(q ; \xi)$ and $D\left(q, q^{\prime} ; \xi\right)$ are the matrices introduced above (see (3.28a) and (3.29a)) and $\widehat{F}(q)$ is the vector

$$
\widehat{F}(q)=\left(\begin{array}{c}
f_{10}(q) \\
f_{01}(q)
\end{array}\right) \in \mathcal{H}_{1}
$$

Lemma 4.1. For sufficiently small e the resolvent $R_{B(\xi)}(w)=\left(B(\xi)-w \widetilde{\mathbb{I}}_{1}\right)^{-1}$ of the operator $B(\xi)$ (here $\widetilde{\mathbb{I}}_{1}$ is the identity operator in $\mathcal{H}_{1}$ ) has the form

$$
\begin{aligned}
\left(R_{B(\xi)}(w) \widehat{F}\right)(q)= & \left(M(q ; \xi)-w \widetilde{\mathbb{I}}_{1}\right)^{-1} \widehat{F}(q) \\
& +\int \frac{K\left(q, q^{\prime} ; \xi ; w\right) \widehat{F}\left(q^{\prime}\right)}{\left(a_{P, 1}(q, \xi)-w\right)\left(a_{P, 1}\left(q^{\prime}, \xi\right)-w\right)} d q^{\prime},
\end{aligned}
$$

where the matrix kernel $K\left(q, q^{\prime} ; \xi ; w\right)$ (as a function of the variables $\left.q, q^{\prime}\right)$ belongs to the space $\mathcal{B}$ introduced above and its norm $\left\|K\left(q, q^{\prime} ; \xi ; w\right)\right\|_{\mathcal{B}}$ is uniformly bounded for all real $\xi<\lambda_{2}^{0}(P)-\varepsilon$ and for $w \in \mathbb{C} \backslash \mathcal{I}(\xi)$. Here $\mathcal{I}(\xi)$ is the cut in $\mathbb{C}$ along the real axis given by

$$
\mathcal{I}(\xi)=\left(\lambda_{1}(P ; \xi), \infty\right) \text {. }
$$

Furthermore, the kernel $K\left(q, q^{\prime} ; \xi ; w\right)$ is a $\mathcal{B}$-valued analytic function of $w \in \mathbb{C} \backslash \mathcal{I}(\xi)$ and its boundary values on the cut $\mathcal{I}(\xi)$, given by

$$
K^{ \pm}\left(q, q^{\prime} ; \xi, x\right)=\lim _{\varepsilon \searrow 0} K\left(q, q^{\prime} ; \xi ; x \pm i \varepsilon\right), \quad x \in \mathcal{I}(\xi),
$$

exist; the limit in (4.4) is understood as the limit with respect to the norm in $\mathcal{B}$. The limit kernel $K^{ \pm}\left(q, q^{\prime} ; \xi, x\right)$ belongs to $\mathcal{B}$ and, as a $\mathcal{B}$-valued function of the variable $x$, 
satisfies, together with its derivatives with respect to $q$ and $q^{\prime}$, a Hölder condition with exponent $1 / 3$, that is,

$$
\left\|K^{ \pm}\left(q, q^{\prime} ; \xi, x+\Delta\right)-K^{ \pm}\left(q, q^{\prime} ; \xi, x\right)\right\|_{\mathcal{B}}<C \Delta^{1 / 3}, \quad 0<\Delta<1,
$$

where $C=C(\xi)$ is a constant independent of $x \in \mathcal{I}(\xi)$.

The proof of this lemma is given in the Appendix.

We will now calculate the resolvent

$$
R_{\mathcal{A}(\xi)}(w)=\left(\mathcal{A}(\xi)-\mathbb{I}_{1} w\right)^{-1},
$$

where $\mathbb{I}_{1}$ is the identity operator in the space $\mathcal{H}^{\leq 1}$. The vector

$$
F=R_{\mathcal{A}(\xi)}(w) G, \quad \text { where } \quad G=\left(g_{00}, \widehat{G}\right) \in \mathcal{H}^{\leq 1}, \quad \widehat{G} \in \mathcal{H}_{1},
$$

is a solution of the equation

$$
\begin{aligned}
\left(a_{00}-w\right) f_{00}+(\widehat{F}, v) & =g_{00} \\
\left(B(\xi)-w \widetilde{\mathbb{I}}_{1}\right) \widehat{F}+f_{00} v & =\widehat{G}
\end{aligned}
$$

where we denote

$$
F=\left(f_{00}, \widehat{F}\right), \quad \widehat{F} \in \mathcal{H}_{1}, \quad v=\left(\begin{array}{l}
v_{10}(q) \\
v_{01}(q)
\end{array}\right) \in \mathcal{H}_{1},
$$

and $(\widehat{F}, v)$ means the scalar product in $\mathcal{H}_{1}$.

This means that

$$
\widehat{F}(q)=\left(\left(B(\xi)-w \widetilde{\mathbb{I}}_{1}\right)^{-1}\left(\widehat{G}-f_{00} v\right)\right)(q) .
$$

Substituting this expression into the first equation in (4.6) we find that

$$
\left(a_{00}-w-\Delta(w)\right) f_{00}=g_{00}-\left(\left(B(\xi)-w \widetilde{\mathbb{I}}_{1}\right)^{-1} \widehat{G}, v\right),
$$

where

$$
\Delta(w)=\left(\left(B(\xi)-w \widetilde{\mathbb{I}}_{1}\right)^{-1} v, v\right)
$$

Thus, if

$$
a_{00}-w-\Delta(w) \neq 0
$$

we obtain that

$$
\begin{aligned}
\left(R_{\mathcal{A}(\xi)}(w) G\right)_{0,0} & =\frac{g_{00}-\left(R_{B(\xi)}(w) G, v\right)}{a_{00}-w-\Delta(w)}, \\
\left(R_{\mathcal{A}(\xi)}(w) G\right)(q) & =R_{B(\xi)}(w)\left(\widehat{G}-\frac{g_{00}-\left(R_{B(\xi)}(w) G, v\right)}{a_{00}-w-\Delta(w)} v\right) .
\end{aligned}
$$

From (4.3) and (4.6a) we find that $\Delta(w)$ is equal to

$$
\Delta(w)=\Delta(w ; \xi)=\int \frac{|v(q)|^{2} d q}{a_{P, 1}(q ; \xi)-w}+\iint \frac{\left(K\left(q, q^{\prime} ; \xi, w\right) v\left(q^{\prime}\right), v(q)\right)}{\left(a_{P, 1}(q ; \xi)-w\right)\left(a_{P, 1}\left(q^{\prime} ; \xi\right)-w\right)} d q d q^{\prime}
$$

Hence, it is clear that the function $\Delta(w)$ is analytic outside the cut $\mathcal{I}(\xi)$ and, since $v(q)$ and $K\left(q, q^{\prime} ; \xi, w\right)$ are smooth, the limits

$$
\Delta^{ \pm}(x)=\lim _{\varepsilon \searrow 0} \Delta(x \pm i \varepsilon)
$$


exist and are equal to

$$
\begin{aligned}
\Delta^{ \pm}(x)= & \int \frac{|v(q)|^{2} d q}{a_{P, 1}(q ; \xi)-(x \pm i 0)} \\
& +\iint \frac{\left(K^{ \pm}\left(q, q^{\prime} ; \xi, x\right) v\left(q^{\prime}\right), v(q)\right)}{\left(a_{P, 1}(q ; \xi)-(x \pm i 0)\right)\left(a_{P, 1}\left(q^{\prime} ; \xi\right)-(x \pm i 0)\right)} d q d q^{\prime} .
\end{aligned}
$$

Furthermore, the functions $\Delta^{ \pm}(x), x \in \mathcal{I}(\xi)$, are Hölder functions.

4.2. The spectrum of the operator $\mathcal{A}(\xi)$. The continuous spectrum of the operator $\mathcal{A}(\xi)$ coincides with the cut $\mathcal{I}(\xi)$, and the eigenvalues $w_{0}=w_{0}(\xi)$ lying outside the cut $\mathcal{I}(\xi)$ are zeros of the equation

$$
a_{00}(\xi)-\Delta\left(w_{0}, \xi\right)=w_{0}, \quad w_{0}<\lambda_{1}(P)=\lambda_{1}(P, \xi) .
$$

It follows from (4.8) and (3.34) that the leading term in $e$ of $\Delta(w)$ is

$$
\Delta(w)=e^{2} \int \frac{c_{1}^{2}(P ; q)+c_{2}^{2}(P ; q)}{a_{P, 1}(q ; \xi)-w} d q+O\left(e^{4}\right) .
$$

It is evident from this expression that for real values of $w<\lambda_{1}(P, \xi)$, as $w$ increases the function $a_{00}-\Delta(w)$ decreases monotonically from the value $a_{00}$ at $w=-\infty$ to the value $a_{00}-\Delta\left(\lambda_{1}(P, \xi)\right)$ at $w=\lambda_{1}(P, \xi)$, as depicted in Figures 1 and 2 , It is evident from these pictures that equation (4.11) has a unique simple root $w_{0}<\lambda_{1}(P, \xi)$ if and only if

$$
a_{00}(\xi)-\Delta\left(\lambda_{1}(P, \xi)\right)<\lambda_{1}(P, \xi) .
$$

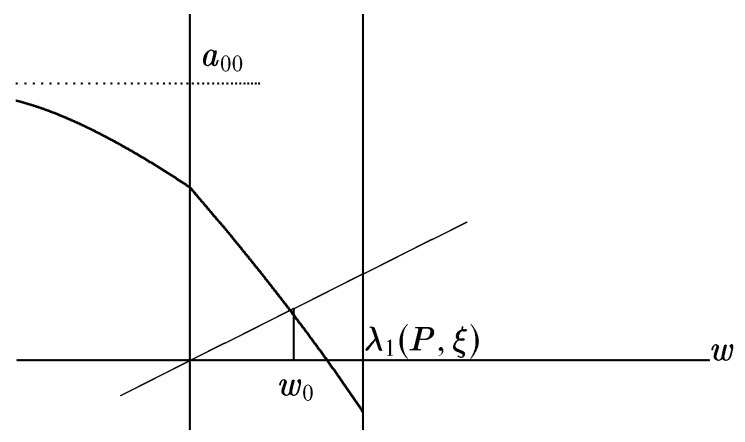

Figure 1. The case $a_{00}(\xi)-\Delta\left(\lambda_{1}(P, \xi)\right)<\lambda_{1}(P, \xi)$

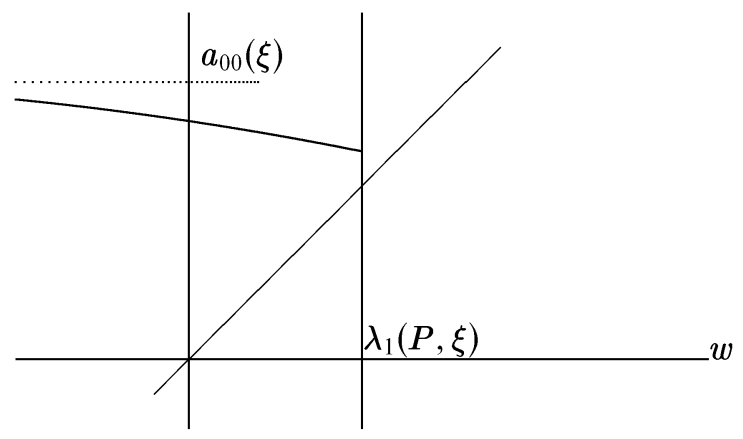

Figure 2. The case $a_{00}(\xi)-\Delta\left(\lambda_{1}(P, \xi)\right) \geq \lambda_{1}(P, \xi)$ 
When the root $w_{0}$ exists, a corresponding eigenvector $F_{0}$ of the operator $\mathcal{A}(\xi)$ (its ground state) has the form (as follows from (4.6a))

$$
F_{0}(\xi)=\left\{f_{00}=1, \widehat{F}_{0}(\xi)\right\}, \quad \text { where } \quad \widehat{F}_{0}(\xi)=R_{B(\xi)}\left(w_{0}\right) v .
$$

It is easy to verify that for a fixed $\xi$, the set of $P \in R^{3}$ for which condition (4.13) holds,

$$
a_{00}(\xi, P)-\Delta\left(\lambda_{1}(P, \xi)\right)<\lambda_{1}(P, \xi),
$$

is a bounded domain $G_{0}(\xi) \subset R^{3}$.

Note that for $w_{0}=\lambda_{1}(P, \xi)$ (that is, for $a_{00}-\Delta\left(\lambda_{1}(P, \xi)=\lambda_{1}(P, \xi)\right)$, at the point $q=q_{0}$ (a minimum point of $\left.a_{P, 1}(q, \xi)\right)$ the vector $\widehat{F}_{0}(q)$ has a singularity $\sim \frac{1}{\left|q-q_{0}\right|^{2}}$ and, consequently, does not belong to the space $\mathcal{H}_{1}$; in other words, if $F_{0}$ is an eigenvector for the operator $\mathcal{A}(\xi)$ corresponding to the eigenvalue $w_{0}$ that happens to lie on the boundary of the continuous spectrum, then it is not a ground state.

Next, it is clear from (4.12) that if $x \in \mathcal{I}(\xi)$, then

$$
\operatorname{Im} \Delta^{ \pm}(x)=\mp e^{2} \pi \int_{a_{P, 1}(q ; \xi)=x}\left(c_{1}^{2}(P, q)+c_{2}^{2}(P, q)\right) d q+O\left(e^{4}\right) \neq 0
$$

for sufficiently small $e$. This means that the operator $\mathcal{A}(\xi)$ has no eigenvalues lying inside the continuous spectrum.

Using the general criterion for when a self-adjoint operator has no singular spectrum (see [24, vol. 4]), it is easy to show that the operator $\mathcal{A}(\xi)$ has no singular spectrum for all $\xi<\lambda_{2}^{0}(P)-\varepsilon$.

Thus,

$$
\mathcal{H}^{\leq 1}= \begin{cases}\mathcal{H}_{a c}(\xi)+\left\{c F_{0}(\xi)\right\} & \text { for } P \in G_{0}(\xi), \\ \mathcal{H}_{a c}(\xi) & \text { for } P \notin G_{0}(\xi) .\end{cases}
$$

Here $\mathcal{H}_{a c}(\xi)$ is the subspace of the absolutely continuous spectrum of $\mathcal{A}(\xi)$, and $\left\{c F_{0}(\xi)\right\}$ is the one-dimensional space spanned by the eigenvector $F_{0}(\xi)$.

4.3. Eigenfunctions of the continuous spectrum of $\mathcal{A}(\xi)$. We denote by $\mathcal{E}$ the operator of embedding $\mathcal{H}_{1}$ into $\mathcal{H}^{\leq 1}$ :

$$
\mathcal{E} \widehat{F}=(0, \widehat{F}) \in \mathcal{H}^{\leq 1}, \quad \widehat{F}=\left(f_{1,0}, f_{0,1}\right) \in \mathcal{H}_{1} .
$$

According to the general criteria of scattering theory (see [26]) the wave operator

$$
W(\xi)=s \cdot \lim _{t \rightarrow+\infty} e\{i t \mathcal{A}(\xi)\} \mathcal{E} e\{-i t M(\xi)\}
$$

exists and is complete; it is a unitary map from the space $\mathcal{H}_{1}$ onto the space $\mathcal{H}_{a c} \subseteq \mathcal{H} \leq 1$ of the absolutely continuous spectrum of $\mathcal{A}(\xi)$. Here $M(\xi)$ is the operator in $\mathcal{H}_{1}$ defined by the matrix $M(q ; \xi)$ (see $(3.28 \mathrm{a})$ ). Furthermore, the operator $W(\xi)$ takes the generalized eigenfunctions of the continuous spectrum of $M(\xi)$,

$$
\left\{F_{\bar{q}, 0}^{\alpha}(q)=\delta(q-\bar{q}) s^{\alpha}, \alpha=(1,0),(0,1), \bar{q} \in R^{3}\right\},
$$

where

$$
s^{1,0}=\left(\begin{array}{l}
1 \\
0
\end{array}\right), \quad s^{0,1}=\left(\begin{array}{l}
0 \\
1
\end{array}\right)
$$

with eigenvalue $a_{P, 1}(\bar{q} ; \xi)$ to eigenvectors $\left\{F_{\bar{q}}^{\alpha}(q), \alpha=(1,0),(0,1), \bar{q} \in R^{3}\right\}$ of the continuous spectrum of $\mathcal{A}(\xi)$ with the same eigenvalues. Following the general formulae of scattering theory (see [26]) we find that

$$
\widetilde{F}_{\bar{q}}^{\alpha}=W(\xi) \widehat{F}_{\bar{q}, 0}^{\alpha}=\lim _{\varepsilon \searrow 0} i \varepsilon R_{\mathcal{A}(\xi)}\left(a_{P, 1}(\bar{q} ; \xi)-i \varepsilon\right) \mathcal{E} \widehat{F}_{\bar{q}, 0}^{\alpha} .
$$

Setting

$$
\widetilde{F}_{\bar{q}}^{\alpha}=\left(f_{00, \bar{q}}^{\alpha}, f_{10, \bar{q}}^{\alpha}(q), f_{01, \bar{q}}^{\alpha}(q)\right)
$$


and using (4.3) and (4.7) we obtain

$$
\begin{aligned}
f_{00, \bar{q}}^{\alpha}= & -\left(a_{00}(\xi)-\Delta^{-}\left(a_{P, 1}(\bar{q} ; \xi)\right)-a_{P, 1}(\bar{q} ; \xi)\right)^{-1} \\
& \times\left[v_{\alpha}(\bar{q})+\int \frac{\left(K^{-}\left(q^{\prime}, \bar{q} ; \xi ; a_{P, 1}(\bar{q} ; \xi)\right) s^{\alpha}, v\left(q^{\prime}\right)\right)}{a_{P, 1}\left(q^{\prime} ; \xi\right)-a_{P, 1}(\bar{q} ; \xi)+i 0} d q^{\prime}\right]
\end{aligned}
$$

(here $($,$) is the scalar product in R^{2}$ ) and

$$
\begin{aligned}
f_{\beta, \bar{q}}^{\alpha}(q)= & \delta(q-\bar{q}) \delta_{\alpha, \beta}+\frac{\left(K^{-}\left(q, \bar{q} ; \xi ; a_{P, 1}(\bar{q} ; \xi)\right) s^{\alpha}\right)_{\beta}}{a_{P, 1}(q ; \xi)-a_{P, 1}(\bar{q} ; \xi)+i 0} \\
& -f_{00, \bar{q}}^{\alpha}\left[v_{\beta}(q)+e^{2} \int \frac{\left(K^{-}\left(q, q^{\prime} ; \xi ; a_{P, 1}(\bar{q} ; \xi)\right) v\left(q^{\prime}\right)\right)_{\beta}}{a_{P, 1}\left(q^{\prime} ; \xi\right)-a_{P, 1}(\bar{q} ; \xi)+i 0} d q^{\prime}\right]
\end{aligned}
$$

where $\beta=(1,0),(0,1)$. In the following lemma we establish that the vectors $\left\{\widetilde{F}_{\bar{q}}^{\alpha}, \alpha=\right.$ $\left.(1,0),(0,1), \bar{q} \in R^{3}\right\}$, which we have constructed, are indeed well-defined generalized functions and any vector $F \in \mathcal{H}_{a c}$ admits an expansion into an integral with respect to the system $\left\{\widetilde{F}_{\bar{q}}^{\alpha}\right\}$.

Lemma 4.2. For any fixed $\xi<\lambda_{2}^{0}(P)-\varepsilon$, the following assertions hold.

1. The component $f_{00, \bar{q}}^{\alpha}=f_{00, \bar{q}}^{\alpha}(\xi)$ of the vector $\widetilde{F}_{\bar{q}}^{\alpha}$ is a bounded continuous function of $\bar{q} \in R^{d}$.

2. For any fixed $\bar{q} \in R^{d}$, the components $f_{10, \bar{q}}^{\alpha}$ and $f_{01, \bar{q}}^{\alpha}$ of the vector $\widetilde{F}_{\bar{q}}^{\alpha}$ belong to the space $S^{\prime}\left(R^{3}\right)$ of generalized functions of moderate growth. Furthermore, for a fixed $q$, each of these components, as a function of $\bar{q}$, is also a generalized function in $S^{\prime}\left(R^{3}\right)$.

3. For each basic function $\varphi \in S\left(R^{3}\right)$ and for each value $\alpha=(1,0),(0,1)$, consider the vector

$$
\psi_{\varphi}^{\alpha}=\left(C_{0,0, \varphi}^{\alpha}, C_{10, \varphi}^{\alpha}(q), C_{01, \varphi}^{\alpha}(q)\right) \in \mathcal{H}^{\leq 1},
$$

where

$$
\begin{aligned}
C_{0,0, \varphi}^{\alpha} & =\int f_{0,0, \bar{q}}^{\alpha} \varphi(\bar{q}) d \bar{q}, \\
C_{10, \varphi}^{\alpha} & =\int f_{10, \bar{q}}^{\alpha} \varphi(\bar{q}) d \bar{q}, \\
C_{01, \varphi}^{\alpha} & =\int f_{01, \bar{q}}^{\alpha} \varphi(\bar{q}) d \bar{q} .
\end{aligned}
$$

Then for any $\varphi_{1}, \varphi_{2} \in S\left(R^{3}\right)$,

$$
\begin{aligned}
\left(\psi_{\varphi_{1}}^{\alpha_{1}}, \psi_{\varphi_{2}}^{\alpha_{2}}\right)_{\mathcal{H} \leq 1}= & C_{0,0, \varphi_{1}}^{\alpha_{1}} \cdot C_{0,0, \varphi_{2}}^{\alpha_{2}} \\
& +\int\left(C_{10, \varphi_{1}}^{\alpha_{1}}(q) \cdot \bar{C}_{10, \varphi_{2}}^{\alpha_{2}}(q)+C_{01, \varphi_{1}}^{\alpha_{1}}(q) \cdot \bar{C}_{01, \varphi_{2}}^{\alpha_{2}}(q)\right) d q \\
= & \delta_{\alpha_{1}, \alpha_{2}}\left(\varphi_{1}, \varphi_{2}\right)_{L_{2}\left(R^{3}\right)} .
\end{aligned}
$$

Consequently, the maps $\varphi \rightarrow \psi_{\varphi}^{\alpha}$, $\alpha=(1,0),(0,1)$, can be extended by continuity to the whole space $L_{2}\left(R^{3}\right)$ and taken together they generate an isometry

$$
W(\xi): \mathcal{H}_{1} \rightarrow \mathcal{H}^{\leq 1}, \quad \operatorname{Ran} W(\xi)=\mathcal{H}_{a c} \subseteq \mathcal{H}^{\leq 1},
$$

which coincides with the wave operator (4.15).

Thus, this lemma gives us license to apply the wave operator (4.15) to the generalized functions (4.16). The proof of this lemma is deferred to the Appendix. 
Corollary. Every vector $F \in \mathcal{H}_{a c}$ admits the expansion

$$
F=F_{f}=\sum_{\alpha} \int f_{\alpha}(\bar{q}) \widetilde{F}_{\bar{q}}^{\alpha} d \bar{q},
$$

where $f=\left\{f_{10}(\bar{q}), f_{01}(\bar{q})\right\} \in \mathcal{H}_{1}$ and $\|F\|_{\mathcal{H} \leq 1}=\|f\|_{\mathcal{H}_{1}}$.

Remark 1. Relation (4.20) can be formally represented as follows:

$$
\begin{aligned}
\left(\widetilde{F}_{\bar{q}_{1}}^{\alpha_{1}}, \widetilde{F}_{\bar{q}_{2}}^{\alpha_{2}}\right)_{\mathcal{H} \leq 1} & =f_{0,0, \bar{q}_{1}}^{\alpha_{1}} \cdot \bar{f}_{0,0, \bar{q}_{2}}^{\alpha_{2}}+\left(f_{10, \bar{q}_{1}}^{\alpha_{1}}, f_{10, \bar{q}_{2}}^{\alpha_{2}}\right)_{L_{2}\left(R^{3}\right)}+\left(f_{01, \bar{q}_{1}}^{\alpha_{1}}, f_{01, \bar{q}_{2}}^{\alpha_{2}}\right)_{L_{2}\left(R^{3}\right)} \\
& =\delta_{\alpha_{1} \alpha_{2}} \delta\left(\bar{q}_{1}-\bar{q}_{2}\right) .
\end{aligned}
$$

Remark 2. Usually generalized eigenvectors of a self-adjoint operator $\mathcal{A}$ acting in a Hilbert space $\mathcal{H}$ are defined as the derivatives

$$
\frac{d E(\lambda) \varphi}{d \sigma_{\varphi}(\lambda)}
$$

where $\varphi \in \mathcal{H}$ is an arbitrary vector, $\{E(\lambda)\}$ is the family of spectral projectors of $\mathcal{A}$, and $\sigma_{\varphi}(\lambda)$ is the spectral measure of the element $\varphi$ (see [6] and [14]). Furthermore, if $\mathcal{A}$ leaves some dense subspace of $\mathcal{H}_{+} \subset \mathcal{H}$ invariant, where the subspace has the structure of a Hilbert space and is nuclearly embedded into $\mathcal{H}$, then for $\varphi \in \mathcal{H}_{+}$the derivative

$$
\frac{d E(\lambda) \varphi}{d \sigma_{\varphi}(\lambda)}=\chi(\lambda)
$$

exists as an element of the dual space $\mathcal{H}_{-}=\mathcal{H}_{+}^{*}$ and is an eigenvector of the operator $\left(\left.\mathcal{A}\right|_{\mathcal{H}_{+}}\right)^{*}$, for the eigenvalue $\lambda$, which is some extension of the operator $\mathcal{A}$. The vector $\chi(\lambda)$ is usually called a generalized eigenvector of $\mathcal{A}$. One can show that the generalized vectors $\widetilde{F}_{\bar{q}}^{\alpha}$ introduced above are generalized eigenvectors of the operator $\mathcal{A}(\xi)$ in this sense.

\section{The LOWER PaRT OF THE SPECTRUM OF THE OPERATOR $H_{P}$}

5.1. The ground state. According to the remark in the Introduction, having found a solution of the equation

$$
w_{0}\left(\xi_{0}\right)=\xi_{0},
$$

where $w_{0}(\xi)$ is an eigenvalue of the operator $\mathcal{A}(\xi)$, and a corresponding eigenvector $F_{1}^{0}\left(\xi_{0}\right) \in \mathcal{H}^{\leq 1}$, we define an eigenvector $F^{0}=\left(F_{1}^{0}, F_{2}^{0}\right), F_{1}^{0} \in \mathcal{H}^{\leq 1}, F_{2}^{0} \in \mathcal{H}^{>1}$, of the operator $H_{P}$ by setting

$$
\begin{aligned}
& F_{1}^{0}=F_{1}^{0}\left(\xi_{0}\right), \\
& F_{2}^{0}=S\left(\xi_{0}\right) F_{1}^{0}\left(\xi_{0}\right) .
\end{aligned}
$$

Here $S\left(\xi_{0}\right)$ is the operator introduced in (3.23) that takes the lower components of the eigenvector $F^{0}$ to its higher components.

Consider the value $\bar{\xi}$ determined from the equation

$$
\bar{\xi}=\widetilde{\lambda}_{1}(P, \bar{\xi}),
$$

where $\widetilde{\lambda}_{1}(P, \xi)$ is the left boundary of the continuous spectrum of the operator $\mathcal{A}(\xi)$ :

$$
\widetilde{\lambda}_{1}(P, \xi)=\min _{q} a_{P, 1}(q, \xi),
$$

which, as is evident from (3.31) and (3.31a), is a monotonically decreasing function of $\xi$ on the interval $-\infty \leq \xi<\lambda_{2}^{0}(P)-\varepsilon$ taking the value $\lambda_{1}^{0}(P)$ at $\xi=-\infty$. Hence equation (5.3) has a unique solution $\bar{\xi}$, which is denoted by $\lambda_{1}(P)$ (see Figure 3). Clearly, $\lambda_{1}(P)<\lambda_{1}^{0}(P)$. 


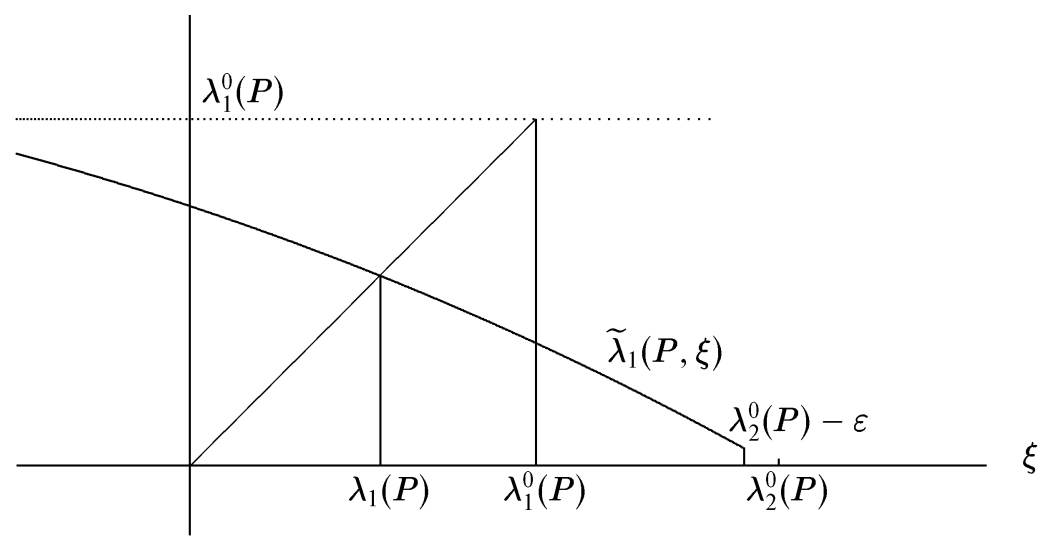

Figure 3 . The graph of the function $\widetilde{\lambda}_{1}(P, \xi)$

Each $\xi<\lambda_{1}(P)$ satisfies the condition $\xi<\widetilde{\lambda}_{1}(P, \xi)$ (as is clear from Figure 3), that is, it lies to the left of the continuous spectrum of $\mathcal{A}(\xi)$. Conversely, if $\xi>\lambda_{1}(P)$, then, again it is clear from Figure 3 that $\xi>\lambda_{1}(P, \xi)$, that is, it lies on the continuous spectrum of $\mathcal{A}(\xi)$. Consequently, a solution $\xi_{0}$ of equation (5.1), being an eigenvalue of the operator $\mathcal{A}\left(\xi_{0}\right)$, lies to the left of $\lambda_{1}(P)$ :

$$
\xi_{0}<\lambda_{1}(P) \text {. }
$$

It is obvious that equation (5.1) is equivalent to equation (4.11) with both $w_{0}$ and $\xi$ replaced by $\xi_{0}$ :

$$
a_{00}\left(P, \xi_{0}\right)-\bar{\Delta}\left(\xi_{0}\right)=\xi_{0},
$$

where $\bar{\Delta}\left(\xi_{0}\right)=\left.\Delta\left(w_{0}, \xi_{0}\right)\right|_{w_{0}=\xi_{0}}$. It is also clear from (3.36a), (4.8) and (4.12) that the left-hand side of equation (5.4) is again a monotonically decreasing function of $\xi_{0}$ on the interval $\left(-\infty, \lambda_{1}(P)\right)$ taking the value $\frac{1}{2} P^{2}$ at $\xi_{0}=-\infty$, and $\frac{1}{2} P^{2}+R\left(\lambda_{1}(P), P\right)$ at $\xi_{0}=\lambda_{1}(P)$, where the quantity $R\left(\xi_{0}, P\right)$ was defined in (3.36a). Thus, as is clear from Figure 4a, equation (5.4) has a unique solution $\xi_{0}=\xi_{0}(P)$ under the condition that

$$
\frac{1}{2} P^{2}+R\left(\lambda_{1}(P), P\right)<\lambda_{1}(P),
$$

and this solution satisfies $\xi_{0}<\lambda_{1}(P)$, as well as $\xi_{0}<\frac{1}{2} P^{2}$. In the case where condition (5.5) is violated, equation (5.4) has no solutions.

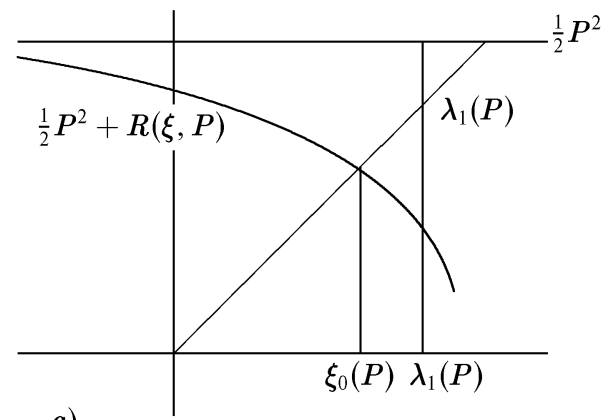

a)

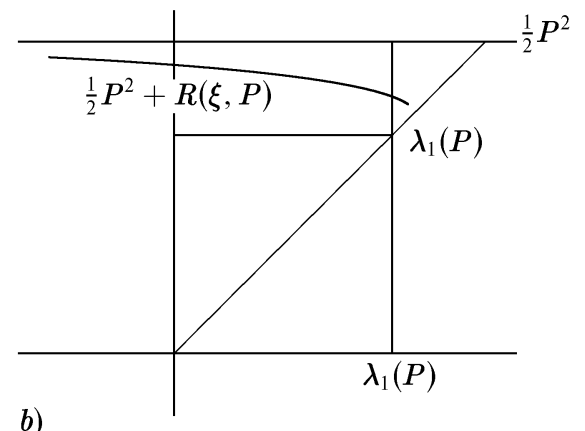

b)

Figure 4. a) $\frac{1}{2} P^{2}+R\left(\lambda_{1}(P) ; P\right)<\lambda_{1}(P) ; \quad$ b) $\frac{1}{2} P^{2}+R\left(\lambda_{1}(P) ; P\right) \geq \lambda_{1}(P)$ 
The domain of the momenta $P$ for which there exists a solution $\xi_{0}(P)$ of equation (5.4) has the form

$$
G_{0}=\left\{P \in R^{3}: \frac{1}{2} P^{2}+R\left(\lambda_{1}(P) ; P\right)<\lambda_{1}(P)\right\} .
$$

Since the quantity $R\left(\lambda_{1}(P) ; P\right)$ is bounded (see (3.36a)) and $\lambda_{1}(P)$ satisfies the inequality

$$
\lambda_{1}(P)<\lambda_{1}^{0}(P)<C_{1}|P|+C_{2},
$$

where $C_{1}, C_{2}>0$ are constants (the last inequality in (5.7) is derived in the same way as (3.5a)), the domain $G_{0}$ is bounded. It is also obvious that $\xi_{0}(P) \rightarrow \lambda_{1}\left(P^{\prime}\right)$ as $P \rightarrow P^{\prime} \in \partial G_{0}$. Thus, we have proved the first assertion of Theorem 1.1

5.2. One-boson subspaces. Now we turn to the second assertion of Theorem 1.1 concerning two one-boson subspaces for the operator $\bar{H}_{P}$.

Since for $\xi<\lambda_{2}^{0}(P)-\varepsilon$ the continuous spectrum of the operator $\mathcal{A}(\xi)$ coincides with the values of the function $a_{P, 1}(q, \xi)$, we should, as we explained in the Introduction and in the preceding subsection, seek the eigenvalues of the continuous spectrum of the operator $\bar{H}_{P}$ from the equation

$$
a_{P, 1}(\bar{q}, \xi)=\xi .
$$

Once we have found its solution $\xi=\xi(\bar{q})$, we will construct the generalized eigenvectors $F_{\bar{q}}^{\alpha}$ of the operator $\bar{H}_{P}$ by setting

$$
F_{\bar{q}}^{\alpha}=F_{\bar{q}, 1}^{\alpha}+F_{\bar{q}, 2}^{\alpha},
$$

where $F_{\bar{q}, 1}^{\alpha}=\widetilde{F}_{\bar{q}}^{\alpha}=\left(f_{0,0, \bar{q}}^{\alpha}, f_{10, \bar{q}}^{\alpha}, f_{01, \bar{q}}^{\alpha}\right)$ are the generalized eigenvectors of the operator $\mathcal{A}\left(\xi(\bar{q})\right.$ ) (see (4.19) ) and the vector $F_{\bar{q}, 2}^{\alpha}$ is determined, purely formally for the moment, by using the equality

$$
F_{\bar{q}, 2}^{\alpha}=S(\xi(\bar{q})) F_{\bar{q}, 1}^{\alpha},
$$

where the operators $\left\{S(\xi), \xi<\lambda_{2}^{0}(P)-\varepsilon\right\}$ were introduced in (3.23) as operators acting from the space $\mathcal{H} \leq 1$ into the space $\mathcal{H}^{>1}$. Below we extend the action of these operators to generalized functions (giving (5.10) a precise meaning), as well as describe a general class of generalized functions containing the vectors $F_{\bar{q}}^{\alpha}$. However, first we analyse equation (5.8).

It follows from (3.31) that if $\bar{q}$ is fixed, the function $a_{P, 1}(\bar{q}, \xi)$ is a monotonically decreasing function of $\xi<\lambda_{2}^{0}(P)-\varepsilon$ taking the value $\frac{1}{2}(P-\bar{q})^{2}+\omega(\bar{q})$ at $\xi=-\infty$ (see Figure 5).

It is clear from this picture that when

$$
\left.a_{P, 1}(\bar{q}, \xi)\right|_{\xi=\lambda_{2}^{0}(P)-\varepsilon}<\lambda_{2}^{0}(P)-\varepsilon,
$$

equation (5.8) has a unique simple root $\xi(\bar{q})<\lambda_{2}^{0}(P)-\varepsilon$ situated on the right of the value $\lambda_{1}(P)$. In the opposite case, when

$$
\left.a_{P, 1}(\bar{q}, \xi)\right|_{\xi=\lambda_{2}^{0}(P)-\varepsilon} \geq \lambda_{2}^{0}(P)-\varepsilon,
$$

there is no such a root. It follows from what we have said above that those $\bar{q} \in R^{3}$ for which there exists a solution $\xi(\bar{q})$ of equation (5.8) form the domain

$$
G_{1, P}^{\varepsilon}=\left\{\bar{q} \in R^{3}:\left.a_{P, 1}(\bar{q}, \xi)\right|_{\xi=\lambda_{2}^{0}(P)-\varepsilon}<\lambda_{2}^{0}(P)-\varepsilon\right\} .
$$

Since the absolute value of the quantity $m(\xi)$ is bounded, this domain is also bounded. Since $a_{P, 1}(\bar{q}, \xi)$ is monotonic as $\xi$ increases, it follows that

$$
G_{1, P}^{\varepsilon_{1}} \subset G_{1, P}^{\varepsilon_{2}} \quad \text { for } \quad \varepsilon_{2}<\varepsilon_{1} .
$$




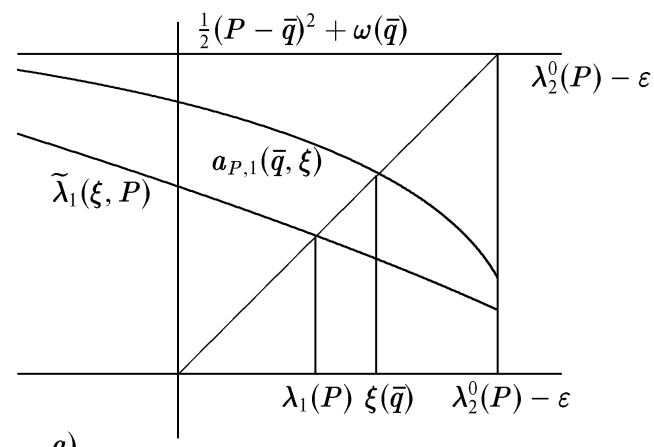

a)

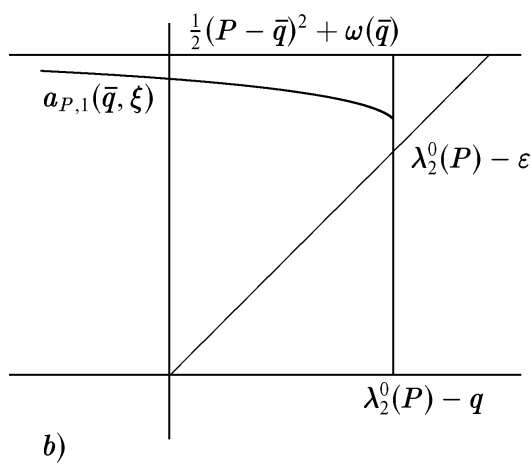

b)

FIGURE 5. a) $\left.\left.a_{P, 1}(\bar{q}, \xi)\right|_{\xi=\lambda_{2}^{0}(P)-\varepsilon}<\lambda_{2}^{0}(P)-\varepsilon ; b\right)\left.a_{P, 1}(\bar{q}, \xi)\right|_{\xi=\lambda_{2}^{0}(P)-\varepsilon} \geq$ $\lambda_{2}^{0}(P)-\varepsilon$

It is easy to verify that the range of the function $\xi(\bar{q})$ for $\bar{q} \in G_{P, 1}^{\varepsilon}$ coincides with the interval $\left(\lambda_{1}(P), \lambda_{2}^{0}(P)-\varepsilon\right)$, and the function $\xi(\bar{q})$ itself is smooth.

We now describe a class of generalized vectors containing the elements $F_{\bar{q}}^{\alpha}$ (see (5.9)) and make relation (5.10) well defined.

Let $\mathcal{L} \subset \mathcal{F}_{\text {sym }}^{\otimes 2}$ be the space of sequences of functions

$$
\Phi=\left\{\varphi_{n_{1}, n_{2}}\left(k_{1}, \ldots, k_{n_{1}} ; \bar{k}_{1}, \ldots, \bar{k}_{n_{2}}\right) ; n_{1}=0,1, \ldots ; n_{2}=0,1, \ldots\right\},
$$

where $\varphi_{0,0} \in \mathbb{C}^{1}$ and $\varphi_{n_{1}, n_{2}}$ is a function of two sets of variables $\left\{k_{i}\right\}_{i=1}^{n_{1}},\left\{\bar{k}_{j}\right\}_{j=1}^{n_{2}}$ (one of which may be empty) that is symmetric with respect to the variables of each set and three times differentiable in each variable so that the function $\varphi_{n_{1}, n_{2}}$ itself and all its derivatives admit the estimate

$$
\left|\left(\prod_{i=1}^{n_{1}} \partial_{k_{i}}^{\alpha_{i}} \prod_{j=1}^{n_{2}} \partial_{\bar{k}_{j}}^{\bar{\alpha}_{j}} \varphi_{n_{1}, n_{2}}\right)\left(k_{1}, \ldots, k_{n_{1}} ; \bar{k}_{1}, \ldots, \bar{k}_{n_{2}}\right)\right|<C_{n_{1}, n_{2}} \prod_{i=1}^{n_{1}} h\left(k_{i}\right) \prod_{j=1}^{n_{2}} h\left(\bar{k}_{j}\right),
$$

where

$$
\begin{array}{ccc}
\alpha_{i}=\left(\alpha_{i}^{(1)}, \alpha_{i}^{(2)}, \alpha_{i}^{(3)}\right), & \alpha_{i}^{m} \leq 3, \quad m=1,2,3, & i=1, \ldots, n_{1} \\
\bar{\alpha}_{j}=\left(\bar{\alpha}_{j}^{(1)}, \bar{\alpha}_{j}^{(2)}, \bar{\alpha}_{j}^{(3)}\right), & \bar{\alpha}_{j}^{m} \leq 3, \quad m=1,2,3, \quad j=1, \ldots, n_{2}
\end{array}
$$

are multi-indices, and $C_{n_{1}, n_{2}}>0$ are constants depending only on the function $\varphi_{n_{1}, n_{2}}$. Furthermore, the set of constants $\left\{C_{n_{1}, n_{2}} ; n_{1}=0,1, \ldots ; n_{2}=0,1, \ldots\right\}$ satisfies the estimate

$$
\sum_{\left\{n_{1}, n_{2}\right\}} \frac{\left|C_{n_{1}, n_{2}}\right|^{2}}{n_{1} ! n_{2} !}\|h\|_{L_{2}\left(R^{3}\right)}^{2\left(n_{1}+n_{2}\right)} \equiv K\left(\left\{C_{n_{1}, n_{2}}\right\}\right)<\infty .
$$

In $\mathcal{L}$ we introduce the norm

$$
\|\Phi\|_{\mathcal{L}}=\inf _{\left\{C_{n_{1}, n_{2}}\right\}} K^{1 / 2}\left(\left\{C_{n_{1}, n_{2}}\right\}\right),
$$

where the infimum is taken over all the sets of constants for which the conditions in (5.13) hold. We observe that the space $\mathcal{L}$ decomposes into the direct sum of spaces

$$
\mathcal{L}=\bigoplus \mathcal{L}_{n_{1}, n_{2}}
$$

where $\mathcal{L}_{0,0}=\mathbb{C}^{1}$ and $\mathcal{L}_{n_{1}, n_{2}}$ consists of the sequences (5.12) in which only the element $\varphi_{n_{1}, n_{2}}$ can be non-zero. The dual space $\mathcal{L}^{*}$ consists of the sequences

$$
F=\left\{f_{n_{1}, n_{2}} ; n_{1}=0,1, \ldots ; n_{2}=0,1, \ldots\right\},
$$


where $f_{0,0} \in \mathbb{C}^{1}$ and $f_{n_{1}, n_{2}} \in S^{1}\left(R^{3\left(n_{1}+n_{2}\right)}\right)$ is a generalized function of moderate growth of the $n_{1}+n_{2}$ variables $\left(k_{1}, \ldots, k_{n_{1}}\right),\left(\bar{k}_{1}, \ldots, \bar{k}_{n_{2}}\right)$ that is symmetric with respect to the variables of each set and such that it is defined on all the functions $\varphi_{n_{1}, n_{2}}$ satisfying condition (5.13). Furthermore, any sequence of functions $f_{n_{1}, n_{2}}$ of the form (5.17) is such that the series

$$
(F, \Phi) \equiv f_{00} \varphi_{00}+\sum_{\substack{n_{1}, n_{2} \\ n_{1}+n_{2}>0}} \frac{f_{n_{1}, n_{2}}\left(\varphi_{n_{1}, n_{2}}\right)}{n_{1} ! n_{2} !}
$$

converges absolutely for all $\Phi \in \mathcal{L}$. Here $f_{n_{1}, n_{2}}\left(\varphi_{n_{1}, n_{2}}\right)$ is the value of a generalized function $f_{n_{1}, n_{2}}$ at a basic function $\left(\varphi_{n_{1}, n_{2}}\right)$. As usual, the norm in $\mathcal{L}^{*}$ is defined by the formula

$$
\|F\|_{\mathcal{L}^{*}}=\sup _{\substack{\Phi \in \mathcal{L} \\\|\Phi\|=1}}(F, \Phi)
$$

As in the case of the space $\mathcal{L}$, the space $\mathcal{L}^{*}$ has a natural decomposition into the direct sum of spaces

$$
\mathcal{L}^{*}=\bigoplus \mathcal{L}_{n_{1}, n_{2}}^{*}
$$

As above, we set

$$
\left(\mathcal{L}^{*}\right)^{\leq n}=\bigoplus_{n_{1}+n_{2} \leq n} \mathcal{L}_{n_{1}, n_{2}}^{*} \quad \text { and } \quad\left(\mathcal{L}^{*}\right)^{>n}=\bigoplus_{n_{1}+n_{2}>n} \mathcal{L}_{n_{1}, n_{2}}^{*} .
$$

Lemma 5.1. 1. The generalized functions

$$
\widetilde{F}_{\bar{q}}^{\alpha}=\left(f_{00, \bar{q}}^{\alpha}, f_{10, \bar{q}}^{\alpha}, f_{01, \bar{q}}^{\alpha}\right)
$$

constructed above belong to the space $\left(\mathcal{L}^{*}\right) \leq 1$.

2. The map $S(\xi):\left(\mathcal{L}^{*}\right)^{\leq 1} \rightarrow\left(\mathcal{L}^{*}\right)^{>1}$ acting according to (3.24):

$$
\begin{aligned}
f_{n_{1}, n_{2}}\left(\left\{k_{i}\right\}_{1}^{n_{1}},\left\{\bar{k}_{j}\right\}_{1}^{n_{2}}\right)= & \sum_{q \in\left\{k_{i}\right\}_{1}^{n_{1}}} b_{10}\left(\left\{k_{i}\right\}_{1}^{n_{1}}, q ;\left\{\bar{k}_{j}\right\}_{1}^{n_{2}}\right) f_{10}(q) \\
& +\sum_{q \in\left\{\bar{k}_{j}\right\}_{1}^{n_{2}}} b_{01}\left(\left\{k_{i}\right\}_{1}^{n_{1}} ;\left\{\bar{k}_{j}\right\}_{1}^{n_{2}}, q\right) f_{01}(q) \\
& +\int d_{10}\left(\left\{k_{i}\right\}_{1}^{n_{1}}, q ;\left\{\bar{k}_{j}\right\}_{1}^{n_{2}}\right) f_{10}(q) d q \\
& +\int d_{01}\left(\left\{k_{i}\right\}_{1}^{n_{1}} ;\left\{\bar{k}_{j}\right\}_{1}^{n_{2}}, q\right) f_{01}(q) d q \\
& +e_{0,0}\left(\left\{k_{i}\right\}_{1}^{n_{1}} ;\left\{\bar{k}_{j}\right\}_{1}^{n_{2}}\right) f_{00}, \quad n_{1}+n_{2}>1,
\end{aligned}
$$

is well defined for elements $\left(f_{00}, f_{10}, f_{01}\right) \in\left(\mathcal{L}^{0}\right)^{\leq 1}$, and the sequences

$$
F=\left\{f_{n_{1}, n_{2}}, n_{1}+n_{2}>1\right\}
$$

belong to $\left(\mathcal{L}^{*}\right)^{>1}$.

3. The vectors $F_{\bar{q}}^{\alpha}$, constructed from the generalized eigenvectors $\widetilde{F}_{\bar{q}}^{\alpha} \in\left(\mathcal{L}^{*}\right)^{\leq 1}$ of the operator $\mathcal{A}(\xi(\bar{q}))$ using formulae (5.9), (5.10), and (5.21), belong to the space $\mathcal{L}^{*}$ and can be represented in the form

$$
F_{\bar{q}}^{\alpha}=\delta_{\bar{q}}^{\alpha}+\overline{\bar{F}}_{\bar{q}}^{\alpha}
$$

where the sequence $\delta_{\bar{q}}^{\alpha}$ has the form

$$
\delta_{\bar{q}}^{\alpha}(q)=\delta(\bar{q}-q) \delta_{\alpha, \beta} \text { for } \beta=(1,0),(0,1), \quad \text { and } \quad\left(\delta_{\bar{q}}^{\alpha}\right)_{n_{1}, n_{2}}=0 \text { for } n_{1}+n_{2} \neq 1 \text {, }
$$


while $\overline{\bar{F}}_{\bar{q}}^{\alpha}$ satisfies the estimate

$$
\left\|\overline{\bar{F}}_{\bar{q}}^{\alpha}\right\|_{\mathcal{L}^{*}}<\operatorname{Meh}(\bar{q})
$$

where $M>0$ is some constant.

The proof of this lemma is given in the Appendix.

Thus, we have constructed the two families

$$
\left\{F_{\bar{q}}^{1,0}, \bar{q} \in G_{1, P}^{\varepsilon}\right\} \quad \text { and } \quad\left\{F_{\bar{q}}^{0,1}, \bar{q} \in G_{1, P}^{\varepsilon}\right\}
$$

of generalized eigenvectors of the operator $\bar{H}_{P}$ with eigenvalues $\xi(\bar{q})=\xi_{P}(\bar{q})$. It is useful to give a formula connecting $\xi_{P}(\bar{q})$ with the eigenvalues $\xi_{P}^{0}$ of the ground states for various $P \in G_{0}$.

Lemma 5.2. The function $\xi_{P}(\bar{q})$ can be represented in the form

$$
\xi_{P}(\bar{q})=\omega(\bar{q})+\xi_{P-\bar{q}}^{0},
$$

where $\xi_{P-\bar{q}}^{0}$ for $P-\bar{q} \in G_{0}$ is the eigenvalue corresponding to the ground state of the operator $\bar{H}_{P-\bar{q}}$.

The proof of this lemma repeats virtually word-for-word the proof of Proposition 4.9 in 1, which is similar, so it is omitted.

Starting from the families (5.25) of generalized eigenfunctions of $\bar{H}_{P}$ we have constructed, we next construct two Hilbert subspaces $\mathcal{H}^{1,0}$ and $\mathcal{H}^{0,1}$ of the space $\mathcal{F}_{\text {sym }}^{\otimes 2}$ that are invariant under the operator $\bar{H}_{P}$.

In $G_{P, 1}^{\varepsilon}$ we consider the level surfaces of the function $\xi(q)$,

$$
\mathcal{X}_{x}=\left\{q \in G_{P, 1}^{\varepsilon} ; \xi(q)=x\right\}, \quad \lambda_{1}(P)<x<\lambda_{2}^{0}(P)-\varepsilon,
$$

and, using the smoothness of this function, on each surface $\mathcal{X}_{x}$ we define a measure $\nu_{x}$ (the so-called Gel'fand-Leray measure; see [3] such that the equality

$$
\int_{G_{P, 1}^{\varepsilon}} \varphi(q) d q=\int_{\lambda_{1}(P)}^{\lambda_{2}^{0}(P)-\varepsilon} d x \int_{\mathcal{X}_{x}} \varphi(q) d \nu_{x}(q)
$$

holds for any function $\varphi(q), q \in G_{1, P}^{\varepsilon}$. Correspondingly, the Hilbert space $L_{2}\left(G_{P, 1}^{\varepsilon}, d q\right)$ can be decomposed into the direct integral

$$
L_{2}\left(G_{P, 1}^{\varepsilon}, d q\right)=\int_{\lambda_{1}(P)}^{\lambda_{2}^{0}(P)-\varepsilon} \mathcal{H}_{x} d x
$$

where $\mathcal{H}_{x}=L_{2}\left(\mathcal{X}_{x}, d \nu_{x}\right)$.

For each infinitely smooth function $\varphi \in \mathcal{D}\left(G_{P, 1}^{\varepsilon}\right)$ with support in $G_{P, 1}^{\varepsilon}$, consider the generalized function

$$
F_{\varphi}^{\alpha}=\int F_{\bar{q}}^{\alpha} \varphi(\bar{q}) d \bar{q} \in \mathcal{L}^{*}
$$

Lemma 5.3. 1. The function $F_{\varphi}^{\alpha}$ belongs to $\mathcal{F}_{\mathrm{sym}}^{\otimes 2}$ for any $\varphi \in \mathcal{D}\left(G_{P, 1}^{\varepsilon}\right)$.

2. There exist two functions $S^{\alpha}(q)$ and $M^{\alpha}\left(q, q^{\prime}\right), q, q^{\prime} \in G_{P, 1}^{\varepsilon}$ such that for any $\varphi_{1}, \varphi_{2} \in \mathcal{D}\left(G_{P, 1}^{\varepsilon}\right)$ the following equality holds:

$$
\begin{aligned}
\left(F_{\varphi^{(1)}}^{\alpha}, F_{\varphi^{(2)}}^{\alpha^{\prime}}\right)_{\mathcal{F}_{\mathrm{sym}}^{\otimes 2}}^{\otimes 2}=\delta_{\alpha, \alpha^{\prime}} & \int_{\lambda_{1}(P)}^{\lambda_{2}^{0}(P)-\varepsilon} d x\left[\int_{\mathcal{X}_{x}}\left(1+S_{x}(q)\right) \varphi_{x}^{(1)}(q) \overline{\varphi_{x}^{(2)}}(q) d \nu_{x}(q)\right. \\
& \left.+\int_{\mathcal{X}_{x}} \int_{\mathcal{X}_{x}} M_{x}\left(q, q^{\prime}\right) \varphi_{x}^{(1)}(q) \overline{\varphi_{x}^{(2)}}\left(q^{\prime}\right) d \nu_{x}(q) d \nu_{x}\left(q^{\prime}\right)\right] .
\end{aligned}
$$


Here $\varphi_{x}^{(1)}, \varphi_{x}^{(2)}, S_{x}$ and $M_{x}$ are the restrictions of the functions $\varphi^{(1)}, \varphi^{(2)}, S$ and $M$ to the surface $\mathcal{X}_{x}$ :

$$
\varphi_{x}^{(1)}(q)=\left.\varphi^{(1)}(q)\right|_{q \in \mathcal{X}_{x}}, \quad M_{x}\left(q, q^{\prime}\right)=\left.M\left(q, q^{\prime}\right)\right|_{\substack{q \in \mathcal{X}_{x} \\ q^{\prime} \in \mathcal{X}_{x}}},
$$

and similarly for $\varphi^{(2)}$ and $S$.

3. The following estimates hold with suitable constants $C_{1}$ and $C_{2}$ :

$$
|S(q)|<C_{1} e \varepsilon^{-1}, \quad\left|M\left(q, q^{\prime}\right)\right|<C_{2} e h(q) h\left(q^{\prime}\right), \quad q, q^{\prime} \in G_{P, 1}^{\varepsilon} .
$$

These estimates imply that for a sufficiently small e we have

$$
F_{\varphi}^{\alpha} \in \mathcal{F}_{\mathrm{sym}}^{\otimes 2} \quad \text { for } \quad \varphi \in L_{2}\left(G_{P, 1}^{\varepsilon}, d q\right)
$$

and

$$
\bar{C}_{2}\|\varphi\|_{L_{2}\left(G_{P, 1}^{\varepsilon}, d q\right)}<\left\|F_{\varphi}^{\alpha}\right\|_{\mathcal{F}_{\mathrm{sym}}^{\otimes 2}}<\bar{C}_{1}\|\varphi\|_{L_{2}\left(G_{P, 1}^{\varepsilon}, d q\right)},
$$

where $\bar{C}_{1}>0, \bar{C}_{2}$ are constants.

The proof of this lemma repeats virtually word-for-word the proof of Lemma 4.10 in [1] and is omitted.

Thus, if we denote the subspace spanned by the vectors $\left\{F_{\varphi}^{\alpha}, \varphi \in L_{2}\left(G_{P, 1}^{\varepsilon}, d q\right)\right\}$ by $\mathcal{H}_{\alpha}^{\varepsilon} \subset \mathcal{F}_{\text {sym }}^{\otimes 2}$, then the spaces $\mathcal{H}_{10}^{\varepsilon}$ and $\mathcal{H}_{01}^{\varepsilon}$ are orthogonal, the map

$$
L_{2}\left(G_{P, 1}^{\varepsilon}, d q\right) \rightarrow \mathcal{H}_{\alpha}^{\varepsilon}, \quad \varphi \rightarrow F_{\varphi}^{\alpha}, \quad \varphi \in L_{2}\left(G_{P, 1}^{\varepsilon}, d q\right),
$$

is continuous and invertible, and the action of the operator $\bar{H}_{P}$ on a vector $F_{\varphi}^{\alpha}$ has the form

$$
\bar{H}_{P} F_{\varphi}^{\alpha}=F_{\bar{\xi} \varphi}^{\alpha}
$$

where $(\bar{\xi} \varphi)(q)=\xi(q) \varphi(q)$.

Furthermore, from (5.11), for $\varepsilon_{2}<\varepsilon_{1}$ we have

$$
\mathcal{H}_{\alpha}^{\varepsilon_{1}} \subset \mathcal{H}_{\alpha}^{\varepsilon_{2}}
$$

and (1.19) obviously holds.

Thus, we have proved the second assertion of Theorem 1.1.

Note that the system of vectors $\left\{F_{\varphi}^{\alpha}, \varphi \in L_{2}\left(G_{P, 1}^{\varepsilon}, d q\right)\right\}$ we have obtained is not orthogonal in $\mathcal{H}_{\alpha}^{\varepsilon}$. The following lemma shows that we can slightly "correct" this system to achieve orthogonality.

Lemma 5.4. There exists a bounded invertible operator $B_{\alpha}$ in $\mathcal{H}_{\alpha}^{\varepsilon}$ commuting with the operator $\left.\bar{H}_{P}\right|_{\mathcal{H}_{\alpha}^{\varepsilon}}$ such that the functions

$$
\widetilde{F}_{\varphi}^{\alpha}=B_{\alpha} F_{\varphi}^{\alpha} \in \mathcal{H}_{\alpha}^{\varepsilon}
$$

satisfy the condition

$$
\left(\widetilde{F}_{\varphi_{1}}^{\alpha}, \widetilde{F}_{\varphi_{2}}^{\alpha}\right)_{\mathcal{H}_{\alpha}^{\varepsilon}}=\left(\varphi_{1}, \varphi_{2}\right)_{L_{2}\left(G_{P, 1}^{\varepsilon}, d q\right)} .
$$

The essence of the proof is that for each level surface $\mathcal{X}_{x}, x \in\left(\lambda_{1}(P), \lambda_{2}^{0}(P)-\varepsilon\right)$ the quadratic form (5.30) considered in $L_{2}\left(\mathcal{X}_{x} d \nu_{x}\right)$ is naturally diagonalizable. See the details in [1].

\section{APPENDIX}

The proofs of the lemmas in the main text largely repeat the proofs of similar lemmas in 11. Therefore we refer the reader to that paper for the details. 
I. Proof of Lemma 3.2, We observe that if the components of the vector

$$
F=\left\{f_{n_{1} n_{2}}, n_{1}+n_{2} \geq 2\right\} \in \mathcal{H}_{>1}
$$

are represented in the form (3.24), then the components of the vector $Q(z) F$ again have an analogous representation with coefficient functions $\widehat{b}_{10}, \widehat{b}_{01}, \widehat{d}_{10}, \widehat{d}_{01}$, and $e$ that have the form

$$
\begin{aligned}
& \widehat{b}_{10}\left(\gamma_{1}, y ; \gamma_{2}\right)=\frac{1}{\frac{1}{2}\left(P-\sum_{k \in \gamma_{1}} k-\sum_{k \in \gamma_{2}} k\right)^{2}+\sum_{k \in \gamma_{1}} \omega(k)+\sum_{k \in \gamma_{2}} \omega(k)-z} \\
& \times\left[-e \sum_{s \in \gamma_{1} \backslash\{y\}} c_{1}\left(P-\sum_{k \in \gamma_{1}} k-\sum_{k \in \gamma_{2}} k ; s\right) b_{10}\left(\gamma_{1} \backslash\{s\}, y ; \gamma_{2}\right)\right. \\
& -e \sum_{s \in \gamma_{2}} c_{2}\left(P-\sum_{k \in \gamma_{1}} k-\sum_{k \in \gamma_{2}} k ; s\right) b_{10}\left(\gamma_{1}, y ; \gamma_{2} \backslash\{s\}\right) \\
& -e \int c_{1}\left(P-\sum_{k \in \gamma_{1}} k-\sum_{k \in \gamma_{2}} k ; t\right) b_{10}\left(\gamma_{1} \cup\{t\}, y ; \gamma_{2}\right) d t \\
& -e \int c_{2}\left(P-\sum_{k \in \gamma_{1}} k-\sum_{k \in \gamma_{2}} k ; t\right) b_{10}\left(\gamma_{1}, y ; \gamma_{2} \cup\{t\}\right) d t \\
& +\frac{e^{2}}{2} \sum_{\substack{s_{1} \in \gamma_{1} \backslash\{y\} \\
s_{2} \in \gamma_{1} \backslash\{y\} \\
s_{1} \neq s_{2}}} w_{1,1}\left(s_{1}, s_{2}\right) b_{10}\left(\gamma_{1} \backslash\left\{s_{1}, s_{2}\right\}, y ; \gamma_{2}\right) \\
& +e^{2} \sum_{\substack{s_{1} \in \gamma_{1} \backslash\{y\} \\
s_{2} \in \gamma_{2}}} w_{1,2}\left(s_{1}, s_{2}\right) b_{10}\left(\gamma_{1} \backslash\left\{s_{1}\right\}, y ; \gamma_{2} \backslash\left\{s_{2}\right\}\right) \\
& +\frac{e^{2}}{2} \sum_{\substack{s_{1} \in \gamma_{2} \\
s_{2} \in \gamma_{2} \\
s_{1} \neq s_{2}}} w_{2,2}\left(s_{1}, s_{2}\right) b_{10}\left(\gamma_{1}, y ; \gamma_{2} \backslash\left\{s_{1}, s_{2}\right\}\right) \\
& +e^{2} \sum_{s \in \gamma_{1} \backslash\{y\}} \int w_{1,1}(s, t) b_{10}\left(\left(\gamma_{1} \backslash\{s\}\right) \cup\{t\}, y ; \gamma_{2}\right) d t \\
& +e^{2} \sum_{s \in \gamma_{2}} \int w_{1,2}(t, s) b_{10}\left(\gamma_{1} \cup\{t\}, y ; \gamma_{2} \backslash\{s\}\right) d t \\
& +e^{2} \sum_{s \in \gamma_{2}} \int w_{1,2}(t, s) b_{10}\left(\gamma_{1} \cup\{t\}, y ; \gamma_{2} \backslash\{s\}\right) d t \\
& +e^{2} \sum_{s \in \gamma_{1} \backslash\{y\}} \int w_{2,1}(s, t) b_{10}\left(\gamma_{1} \backslash\{s\}, y ; \gamma_{2} \cup\{t\}\right) d t \\
& +e^{2} \sum_{s \in \gamma_{2}} \int w_{2,2}(s, t) b_{10}\left(\gamma_{1}, y ;\left(\gamma_{2} \backslash\{s\}\right) \cup\{t\}\right) d t \\
& +\frac{e^{2}}{2} \iint w_{1,1}\left(t_{1}, t_{2}\right) b_{10}\left(\gamma_{1} \cup\left\{t_{1}, t_{2}\right\}, y ; \gamma_{2}\right) d t_{1} d t_{2} \\
& +e^{2} \iint w_{1,2}\left(t_{1}, t_{2}\right) b_{10}\left(\gamma_{1} \cup\left\{t_{1}\right\}, y ; \gamma_{2} \cup\left\{t_{2}\right\}\right) d t_{1} d t_{2} \\
& \left.+\frac{e^{2}}{2} \iint w_{2,2}\left(t_{1}, t_{2}\right) b_{10}\left(\gamma_{1}, y ; \gamma_{2} \cup\left\{t_{1}, t_{2}\right\}\right) d t_{1} d t_{2}\right] ;
\end{aligned}
$$




$$
\begin{aligned}
& \widehat{b}_{01}\left(\gamma_{1} ; \gamma_{2}, x\right)=\frac{1}{\frac{1}{2}\left(P-\sum_{k \in \gamma_{1}} k-\sum_{k \in \gamma_{2}} k\right)^{2}+\sum_{k \in \gamma_{1}} \omega(k)+\sum_{k \in \gamma_{2}} \omega(k)-z} \\
& \times\left[-e \sum_{s \in \gamma_{1}} c_{1}\left(P-\sum_{k \in \gamma_{1}} k-\sum_{k \in \gamma_{2}} k ; s\right) b_{01}\left(\gamma_{1} \backslash\{s\} ; \gamma_{2}, x\right)\right. \\
& -e \sum_{s \in \gamma_{2} \backslash\{x\}} c_{2}\left(P-\sum_{k \in \gamma_{1}} k-\sum_{k \in \gamma_{2}} k ; s\right) b_{01}\left(\gamma_{1} ; \gamma_{2} \backslash\{s\}, x\right) \\
& -e \int c_{1}\left(P-\sum_{k \in \gamma_{1}} k-\sum_{k \in \gamma_{2}} k ; t\right) b_{01}\left(\gamma_{1} \cup\{t\} ; \gamma_{2}, x\right) d t \\
& -e \int c_{2}\left(P-\sum_{k \in \gamma_{1}} k-\sum_{k \in \gamma_{2}} k ; t\right) b_{01}\left(\gamma_{1} ; \gamma_{2} \cup\{t\}, x\right) d t \\
& +\frac{e^{2}}{2} \sum_{\substack{s_{1} \in \gamma_{1} \\
s_{2} \in \gamma_{2} \\
s_{1} \neq s_{2}}} w_{1,1}\left(s_{1}, s_{2}\right) b_{01}\left(\gamma_{1} \backslash\left\{s_{1}, s_{2}\right\} ; \gamma_{2}, x\right) \\
& +\sum_{\substack{s_{1} \in \gamma_{1} \\
s_{2} \in \gamma_{2} \backslash\{x\}}} w_{1,2}\left(s_{1}, s_{2}\right) b_{01}\left(\gamma_{1} \backslash\left\{s_{1}\right\} ; \gamma_{2} \backslash\left\{s_{2}\right\}, x\right) \\
& +\frac{e^{2}}{2} \sum_{\substack{s_{1} \in \gamma_{2} \backslash\{x\} \\
s_{2} \in \gamma_{2} \backslash\{x\} \\
s_{1} \neq s_{2}}} w_{2,2}\left(s_{1}, s_{2}\right) b_{01}\left(\gamma_{1} ; \gamma_{2} \backslash\left\{s_{1}, s_{2}\right\}, x\right) \\
& +e^{2} \sum_{s \in \gamma_{1}} \int w_{1,1}(s, t) b_{01}\left(\left(\gamma_{1} \backslash\{s\}\right) \cup\{t\} ; \gamma_{2}, x\right) d t \\
& +e^{2} \sum_{s \in \gamma_{2} \backslash\{x\}} \int w_{1,2}(t, s) b_{01}\left(\gamma_{1} \cup\{t\} ; \gamma_{2} \backslash\{s\}, x\right) d t \\
& +e^{2} \sum_{s \in \gamma_{1}} \int w_{2,1}(s, t) b_{01}\left(\gamma_{1} \backslash\{s\} ; \gamma_{2} \cup\{t\}, x\right) d t \\
& +e^{2} \sum_{s \in \gamma_{2} \backslash\{x\}} \int w_{2,2}(s, t) b_{01}\left(\gamma_{1} ;\left(\gamma_{2} \backslash\{s\}\right) \cup\{t\}, x\right) d t \\
& +\frac{e^{2}}{2} \iint w_{1,1}\left(t_{1}, t_{2}\right) b_{01}\left(\gamma_{1} \cup\left\{t_{1}, t_{2}\right\} ; \gamma_{2}, x\right) d t_{1} d t_{2} \\
& +e^{2} \iint w_{1,2}\left(t_{1}, t_{2}\right) b_{01}\left(\gamma_{1} \cup\left\{t_{1}\right\} ; \gamma_{2} \cup\left\{t_{2}\right\}, x\right) d t_{1} d t_{2} \\
& \left.+\frac{e^{2}}{2} \iint w_{2,2}\left(t_{1}, t_{2}\right) b_{01}\left(\gamma_{1} ; \gamma_{2} \cup\left\{t_{1}, t_{2}\right\}, x\right) d t_{1} d t_{2}\right] ;
\end{aligned}
$$




$$
\begin{aligned}
& \widehat{d}_{10}\left(\gamma_{1}, y ; \gamma_{2}\right)=\frac{1}{\frac{1}{2}\left(P-\sum_{k \in \gamma_{1}} k-\sum_{k \in \gamma_{2}} k\right)^{2}+\sum_{k \in \gamma_{1}} \omega(k)+\sum_{k \in \gamma_{2}} \omega(k)-z} \\
& \times\left[-e \sum_{s \in \gamma_{1}} c_{1}\left(P-\sum_{k \in \gamma_{1}} k-\sum_{k \in \gamma_{2}} k ; s\right) d_{10}\left(\gamma_{1} \backslash\{s\}, y ; \gamma_{2}\right)\right. \\
& -e \sum_{s \in \gamma_{2}} c_{2}\left(P-\sum_{k \in \gamma_{1}} k-\sum_{k \in \gamma_{2}} k ; s\right) d_{10}\left(\gamma_{1}, y ; \gamma_{2} \backslash\{s\}\right) \\
& -e \int c_{1}\left(P-\sum_{k \in \gamma_{1}} k-\sum_{k \in \gamma_{2}} k ; t\right) d_{10}\left(\gamma_{1} \cup\{t\}, y ; \gamma_{2}\right) d t \\
& -e \int c_{2}\left(P-\sum_{k \in \gamma_{1}} k-\sum_{k \in \gamma_{2}} k ; t\right) d_{10}\left(\gamma_{1}, y ; \gamma_{2} \cup\{t\}\right) d t \\
& -e c_{1}\left(P-\sum_{k \in \gamma_{1}} k-\sum_{k \in \gamma_{2}} k ; y\right) b_{10}\left(\gamma_{1} \cup\{y\}, y ; \gamma_{2}\right) \\
& +\frac{e^{2}}{2} \sum_{\substack{s_{1} \in \gamma_{1} \\
s_{2} \in \gamma_{2} \\
s_{1} \neq s_{2}}} w_{1,1}\left(s_{1}, s_{2}\right) d_{10}\left(\gamma_{1} \backslash\left\{s_{1}, s_{2}\right\}, y ; \gamma_{2}\right) \\
& +e^{2} \sum_{\substack{s_{1} \in \gamma_{1} \\
s_{2} \in \gamma_{2}}} w_{1,2}\left(s_{1}, s_{2}\right) d_{10}\left(\gamma_{1} \backslash\left\{s_{1}\right\}, y ; \gamma_{2} \backslash\left\{s_{2}\right\}\right) \\
& +\frac{e^{2}}{2} \sum w_{2,2}\left(s_{1}, s_{2}\right) d_{10}\left(\gamma_{1}, y ; \gamma_{2} \backslash\left\{s_{1}, s_{2}\right\}\right) \\
& +e^{2} \sum_{s \in \gamma_{1}} \int w_{1,1}(s, t) d_{10}\left(\left(\gamma_{1} \backslash\{s\}\right) \cup\{t\}, y ; \gamma_{2}\right) d t \\
& +e^{2} \sum_{s \in \gamma_{2}} \int w_{1,2}(t, s) d_{10}\left(\gamma_{1} \cup\{t\}, y ; \gamma_{2} \backslash\{s\}\right) d t \\
& +e^{2} \sum_{s \in \gamma_{1}} \int w_{2,1}(s, t) d_{10}\left(\gamma_{1} \backslash\{s\}, y ; \gamma_{2} \cup\{t\}\right) d t \\
& +e^{2} \sum_{s \in \gamma_{2}} \int w_{2,2}(s, t) d_{10}\left(\gamma_{1}, y ;\left(\gamma_{2} \backslash\{s\}\right) \cup\{t\}\right) d t \\
& +e^{2} \sum_{s \in \gamma_{2}} w_{1,2}(y, s) b_{10}\left(\gamma_{1} \cup\{y\} ; y ; \gamma_{2} \backslash\{s\}\right) \\
& +e^{2} \sum_{s \in \gamma_{1} \backslash\{y\}} w_{1,1}(s, y) b_{10}\left(\left(\gamma_{1} \backslash\{s\}\right) \cup\{y\}, y ; \gamma_{2}\right) \\
& +\frac{e^{2}}{2} \iint w_{1,1}\left(t_{1}, t_{2}\right) d_{10}\left(\gamma_{1} \cup\left\{t_{1}, t_{2}\right\}, y ; \gamma_{2}\right) d t_{1} d t_{2} \\
& +e^{2} \iint w_{1,2}\left(t_{1}, t_{2}\right) d_{10}\left(\gamma_{1} \cup\left\{t_{1}\right\}, y ; \gamma_{2} \cup\left\{t_{2}\right\}\right) d t_{1} d t_{2} \\
& +\frac{e^{2}}{2} \iint w_{2,2}\left(t_{1}, t_{2}\right) d_{10}\left(\gamma_{1}, y ; \gamma_{2} \cup\left\{t_{1}, t_{2}\right\}\right) d t_{1} d t_{2} \\
& +e^{2} \int w_{1,1}(t, y) b_{10}\left(\gamma_{1} \cup\{t, y\}, y ; \gamma_{2}\right) d t \\
& \left.+\frac{e^{2}}{2} \int w_{1,2}(y, t) b_{10}\left(\gamma_{1} \cup\{y\}, y ; \gamma_{2} \cup\{t\}\right) d t\right] ;
\end{aligned}
$$




$$
\begin{aligned}
& \widehat{d}_{01}\left(\gamma_{1} ; \gamma_{2}, x\right)=\frac{1}{\frac{1}{2}\left(P-\sum_{k \in \gamma_{1}} k-\sum_{k \in \gamma_{2}} k\right)^{2}+\sum_{k \in \gamma_{1}} \omega(k)+\sum_{k \in \gamma_{2}} \omega(k)-z} \\
& \times\left[-e \sum_{s \in \gamma_{1}} c_{1}\left(P-\sum_{k \in \gamma_{1}} k-\sum_{k \in \gamma_{2}} k ; s\right) d_{01}\left(\gamma_{1} \backslash\{s\} ; \gamma_{2}, x\right)\right. \\
& -e \sum_{s \in \gamma_{2}} c_{2}\left(P-\sum_{k \in \gamma_{1}} k-\sum_{k \in \gamma_{2}} k ; s\right) d_{01}\left(\gamma_{1} ; \gamma_{2} \backslash\{s\}, x\right) \\
& -e \int c_{1}\left(P-\sum_{k \in \gamma_{1}} k-\sum_{k \in \gamma_{2}} k ; t\right) d_{01}\left(\gamma_{1} \cup\{t\} ; \gamma_{2}, x\right) d t \\
& -e \int c_{2}\left(P-\sum_{k \in \gamma_{1}} k-\sum_{k \in \gamma_{2}} k ; t\right) d_{01}\left(\gamma_{1} ; \gamma_{2} \cup\{t\}, x\right) d t \\
& -e c_{2}\left(P-\sum_{k \in \gamma_{1}} k-\sum_{k \in \gamma_{2}} k ; x\right) d_{01}\left(\gamma_{1} ; \gamma_{2} \cup\{x\}, x\right) \\
& +\frac{e^{2}}{2} \sum_{\substack{s_{1} \in \gamma_{1} \\
s_{2} \in \gamma_{1} \\
s_{1} \neq s_{2}}} w_{1,1}\left(s_{1}, s_{2}\right) d_{01}\left(\gamma_{1} \backslash\left\{s_{1}, s_{2}\right\} ; \gamma_{2}, x\right) \\
& +e^{2} \sum_{\substack{s_{1} \in \gamma_{1} \\
s_{2} \in \gamma_{2}}} w_{1,2}\left(s_{1}, s_{2}\right) d_{01}\left(\gamma_{1} \backslash\left\{s_{1}\right\} ; \gamma_{2} \backslash\left\{s_{2}\right\}, x\right) \\
& +\frac{e^{2}}{2} \sum_{\substack{s_{1} \in \gamma_{2} \\
s_{2} \in \gamma_{2} \\
s_{1} \neq s_{2}}} w_{2,2}\left(s_{1}, s_{2}\right) d_{01}\left(\gamma_{1} ; \gamma_{2} \backslash\left\{s_{1}, s_{2}\right\}, x\right) \\
& +e^{2} \sum_{s \in \gamma_{1}} \int w_{1,1}(t, s) d_{01}\left(\left(\gamma_{1} \backslash\{s\}\right) \cup\{t\} ; \gamma_{2}, x\right) d t \\
& +e^{2} \sum_{s \in \gamma_{2}} \int w_{1,2}(t, s) d_{01}\left(\gamma_{1} \cup\{t\} ; \gamma_{2} \backslash\{s\}, x\right) d t \\
& +e^{2} \sum_{s \in \gamma_{1}} \int w_{2,1}(s, t) d_{01}\left(\gamma_{1} \backslash\{s\} ; \gamma_{2} \cup\{t\}, x\right) d t \\
& +e^{2} \sum_{s \in \gamma_{2}} \int w_{2,2}(s, t) d_{01}\left(\gamma_{1} ;\left(\gamma_{2} \backslash\{s\}\right) \cup\{t\}, x\right) d t \\
& +\frac{e^{2}}{2} \iint w_{1,1}\left(t_{1}, t_{2}\right) d_{01}\left(\gamma_{1} \cup\left\{t_{1}, t_{2}\right\} ; \gamma_{2}, x\right) d t_{1} d t_{2} \\
& +e^{2} \iint w_{1,2}\left(t_{1}, t_{2}\right) d_{01}\left(\gamma_{1} \cup\left\{t_{1}\right\} ; \gamma_{2} \cup\left\{t_{2}\right\}, x\right) d t_{1} d t_{2} \\
& +\frac{e^{2}}{2} \iint w_{2,2}\left(t_{1}, t_{2}\right) d_{01}\left(\gamma_{1} ; \gamma_{2} \cup\left\{t_{1}, t_{2}\right\}, x\right) d t_{1} d t_{2} \\
& +e^{2} \sum_{s \in \gamma_{1}} w_{2,1}(s, x) b_{01}\left(\gamma_{1} \backslash\{s\} ; \gamma_{2} \cup\{x\}, x\right) \\
& +e^{2} \sum_{s \in \gamma_{2}} w_{2,2}(s, x) b_{01}\left(\gamma_{1} ;\left(\gamma_{2} \backslash\{s\}\right) \cup\{x\}, x\right) \\
& +\frac{e^{2}}{2} \int w_{1,2}(t, x) b_{01}\left(\gamma_{1} \cup\{t\} ; \gamma_{2} \cup\{x\}, x\right) d t \\
& \left.+e^{2} \int w_{2,2}(t, x) b_{01}\left(\gamma_{1} \cup\{t\} ; \gamma_{2} \cup\{x\}, x\right) d t\right] ;
\end{aligned}
$$


THE HAMILTONIAN OF THE SPINLESS PAULI-FIERZ MODEL

$$
\begin{aligned}
&\left.\widehat{e}_{0,0}\left(\gamma_{1} ; \gamma_{2}\right)=\frac{1}{\frac{1}{2}(} P-\sum_{k \in \gamma_{1}} k-\sum_{k \in \gamma_{2}} k\right)^{2}+\sum_{k \in \gamma_{1}} \omega(k)+\sum_{k \in \gamma_{2}} \omega(k)-z \\
& \times\left[-e \sum_{s \in \gamma_{1}} c_{1}\left(P-\sum_{k \in \gamma_{1}} k-\sum_{k \in \gamma_{2}} k ; s\right) e_{00}\left(\gamma_{1} \backslash\{s\} ; \gamma_{2}\right)\right. \\
& \quad-e \sum_{s \in \gamma_{2}} c_{2}\left(P-\sum_{k \in \gamma_{1}} k-\sum_{k \in \gamma_{2}} k ; s\right) e_{00}\left(\gamma_{1} ; \gamma_{2} \backslash\{s\}\right) \\
&+e \int c_{1}\left(P-\sum_{k \in \gamma_{1}} k-\sum_{k \in \gamma_{2}} k ; t\right) e_{00}\left(\gamma_{1} \cup\{t\} ; \gamma_{2}\right) d t \\
& \quad-e \int c_{2}\left(P-\sum_{k \in \gamma_{1}} k-\sum_{k \in \gamma_{2}} k ; t\right) e_{00}\left(\gamma_{1} ; \gamma_{2} \cup\{t\}\right) d t \\
&+\frac{e^{2}}{2} \sum_{s_{1} \in \gamma_{1}} w_{1,1}\left(s_{1}, s_{2}\right) e_{00}\left(\gamma_{1} \backslash\left\{s_{1}, s_{2}\right\} ; \gamma_{2}\right) \\
&+e_{s_{2} \in \gamma_{2}} \int e_{s_{1}} \int s_{2} w_{1,2}\left(t_{1}, t_{2}\right) e_{00}\left(\gamma_{1} \cup\left\{t_{1}\right\} ; \gamma_{2} \cup\left\{t_{2}\right\}\right) d t_{1} d t_{2} \\
&+e^{2} \sum_{s_{1} \in \gamma_{1}} w_{1,2}\left(s_{1}, s_{2}\right) e_{00}\left(\gamma_{1} \backslash\left\{s_{1}\right\} ; \gamma_{2} \backslash\left\{s_{2}\right\}\right) \\
&+w_{2} \in \gamma_{2}
\end{aligned}
$$


It is clear from formulae (3.3a)-(3.3g) that the vector $G F_{1}$ has the form (3.24) with the non-zero coefficient functions

(A.I.6)

$$
\begin{aligned}
& b_{10}^{0}\left(\left\{k_{1}, k_{2}\right\}, y, \varnothing\right)=\frac{e c_{1}\left(P-k_{1}-k_{2} ; y\right)}{\frac{1}{2}\left(P-k_{1}-k_{2}\right)^{2}+\omega\left(k_{1}\right)+\omega\left(k_{2}\right)-z} ; \\
& b_{10}^{0}\left(\left\{k_{1}\right\}, y ;\left\{k_{2}\right\}\right)=\frac{e c_{2}\left(P-k_{1}-k_{2} ; y\right)}{\frac{1}{2}\left(P-k_{1}-k_{2}\right)^{2}+\omega\left(k_{1}\right)+\omega\left(k_{2}\right)-z} ; \\
& b_{10}^{0}\left(\left\{k_{1}, k_{2}, k_{3}\right\}, y ; \varnothing\right)=\frac{-\frac{e^{2}}{2} w_{1,1}\left(\left\{k_{1}, k_{2}, k_{3}\right\} \backslash\{y\}\right)}{\frac{1}{2}\left(P-k_{1}-k_{2}-k_{3}\right)^{2}+\omega\left(k_{1}\right)+\omega\left(k_{2}\right)+\omega\left(k_{3}\right)-z}, \\
& y \in\left\{k_{1}, k_{2}, k_{3}\right\} \\
& b_{10}^{0}\left(\left\{k_{1}, k_{2}\right\}, y ;\{k\}\right)=\frac{-\frac{e^{2}}{2} w_{1,2}\left(\left\{k_{1}, k_{2}\right\} \backslash\{y\}, k\right)}{\frac{1}{2}\left(P-k_{1}-k_{2}-k\right)^{2}+\omega\left(k_{1}\right)+\omega\left(k_{2}\right)+\omega(k)-z}, \\
& y \in\left\{k_{1}, k_{2}\right\} ; \\
& b_{10}^{0}\left(\{k\} ; y ;\left\{k_{1}, k_{2}\right\}\right)=\frac{-\frac{e^{2}}{2} w_{2,2}\left(k_{1}, k_{2}\right)}{\frac{1}{2}\left(P-k_{1}-k_{2}-k\right)^{2}+\omega\left(k_{1}\right)+\omega\left(k_{2}\right)+\omega(k)-z} ; \\
& b_{01}^{0}\left(\varnothing ;\left\{k_{1}, k_{2}\right\}, x\right)=\frac{e c_{2}\left(P-k_{1}-k_{2} ; x\right)}{\frac{1}{2}\left(P-k_{1}-k_{2}\right)^{2}+\omega\left(k_{1}\right)+\omega\left(k_{2}\right)-z} ; \\
& b_{01}^{0}\left(\left\{k_{1}\right\},\left\{k_{2}\right\}, x\right)=\frac{e c_{1}\left(P-k_{1}-k_{2} ; x\right)}{\frac{1}{2}\left(P-k_{1}-k_{2}\right)^{2}+\omega\left(k_{1}\right)+\omega\left(k_{2}\right)-z} ; \\
& b_{01}^{0}\left(\varnothing ;\left\{k_{1}, k_{2}, k_{3}\right\}, x\right)=\frac{-\frac{e^{2}}{2} w_{2,1}\left(\left\{k_{1}, k_{2}, k_{3}\right\} \backslash\{x\}\right)}{\frac{1}{2}\left(P-k_{1}-k_{2}-k_{3}\right)^{2}+\omega\left(k_{1}\right)+\omega\left(k_{2}\right)+\omega\left(k_{3}\right)-z}, \\
& x \in\left\{k_{1}, k_{2}, k_{3}\right\} ; \\
& b_{01}^{0}\left(\left\{k_{1}, k_{2}\right\} ;\{k\}, x\right)=\frac{-\frac{e^{2}}{2} w_{1,1}\left(k_{1}, k_{2}\right)}{\frac{1}{2}\left(P-k_{1}-k_{2}-k\right)^{2}+\omega\left(k_{1}\right)+\omega\left(k_{2}\right)+\omega(k)-z}, \\
& x \in\left\{k_{1}, k_{2}\right\} \\
& b_{01}^{0}\left(\{k\} ;\left\{k_{1}, k_{2}\right\}, x\right)=\frac{-\frac{e^{2}}{2} w_{1,2}\left(k,\left\{k_{1}, k_{2}\right\} \backslash\{x\}\right)}{\frac{1}{2}\left(P-k_{1}-k_{2}-k\right)^{2}+\omega\left(k_{1}\right)+\omega\left(k_{2}\right)+\omega(k)-z} ; \\
& e_{00}^{0}\left(\left\{k_{1}, k_{2}\right\}, \varnothing\right)=\frac{-\frac{e^{2}}{2} w_{1,1}\left(k_{1}, k_{2}\right)}{\frac{1}{2}\left(P-k_{1}-k_{2}\right)^{2}+\omega\left(k_{1}\right)+\omega\left(k_{2}\right)-z} ; \\
& e_{00}^{0}\left(\varnothing,\left\{k_{1}, k_{2}\right\}\right)=\frac{-\frac{e^{2}}{2} w_{2,2}\left(k_{1}, k_{2}\right)}{\frac{1}{2}\left(P-k_{1}-k_{2}\right)^{2}+\omega\left(k_{1}\right)+\omega\left(k_{2}\right)-z} ; \\
& e_{00}^{0}\left(\left\{k_{1}\right\},\left\{k_{2}\right\}\right)=\frac{-\frac{e^{2}}{2} w_{1,2}\left(k_{1}, k_{2}\right)}{\frac{1}{2}\left(P-k_{1}-k_{2}\right)^{2}+\omega\left(k_{1}\right)+\omega\left(k_{2}\right)-z} .
\end{aligned}
$$

Now consider the space $\mathcal{M}$ of tuples of functions

$$
\mu=\left(b_{10}\left(\gamma_{1}, y ; \gamma_{2}\right), b_{01}\left(\gamma_{1} ; \gamma_{2}, x\right), d_{10}\left(\gamma_{1}, y ; \gamma_{2}\right), d_{01}\left(\gamma_{1} ; \gamma_{2}, x\right), e_{00}\left(\gamma_{1}, \gamma_{2}\right)\right)
$$


where $\gamma_{1}, \gamma_{2} \in \Gamma_{0} ; y \in \gamma_{1}$ in the case of $b_{10} ; x \in \gamma_{2}$ in the case of $b_{01} ; y \notin \gamma_{1}$ and $x \notin \gamma_{2}$ in the cases of $d_{10}$ and $d_{01}$, respectively; and the tuples satisfy the estimates

$$
\begin{aligned}
\left|b_{10}\left(\gamma_{1}, y ; \gamma_{2}\right)\right|<A \prod_{q \in \gamma_{1} \backslash\{y\}} h(q) \cdot \prod_{t \in \gamma_{2}} h(t)\left(\left|\gamma_{1}\right| !\left|\gamma_{2}\right| !\right)^{1 / 2}, \\
b_{01}\left(\gamma_{1} ; \gamma_{2}, x\right)<A \prod_{q \in \gamma_{1}} h(q) \cdot \prod_{t \in \gamma_{2} \backslash\{x\}} h(t)\left(\left|\gamma_{1}\right| !\left|\gamma_{2}\right| !\right)^{1 / 2}, \\
d_{10}\left(\gamma_{1}, y ; \gamma_{2}\right)<A \prod_{q \in \gamma_{1} \cup\{y\}} h(q) \cdot \prod_{t \in \gamma_{2}} h(t)\left(\left|\gamma_{1}\right| !\left|\gamma_{2}\right| !\right)^{1 / 2}, \\
d_{01}\left(\gamma_{1} ; \gamma_{2}, x\right)<A \prod_{q \in \gamma_{1}} h(q) \cdot \prod_{t \in \gamma_{2} \cup\{x\}} h(t)\left(\left|\gamma_{1}\right| !\left|\gamma_{2}\right| !\right)^{1 / 2}, \\
e_{00}\left(\gamma_{1}, \gamma_{2}\right)<A \prod_{q \in \gamma_{1}} h(q) \cdot \prod_{t \in \gamma_{2}} h(t)\left(\left|\gamma_{1}\right| !\left|\gamma_{2}\right| !\right)^{1 / 2},
\end{aligned}
$$

where the constant $A>0$ depends only on the whole tuple (A.I.7). After $\mathcal{M}$ is equipped with the norm

$$
\|\mu\|=\inf A,
$$

where the infimum is taken over all the $A$ for which the estimates (A.I.8) hold, the space $\mathcal{M}$ becomes a Banach space. From the estimates (3.5) and (3.7) we find that the tuple (A.I.6)

$$
\mu_{0}=\left\{b_{10}^{0}, b_{01}^{0}, d_{10}^{0}, d_{01}^{0}, e_{00}^{0}\right\}
$$

belongs to the space $\mathcal{M}$ and

$$
\left\|\mu_{0}\right\|<e\left(\frac{C_{1}}{\lambda_{2}^{0}(P)-z}+C_{2}\right)
$$

where $C_{1}>0, C_{2}>0$ are constants.

We now consider the operator $\mathcal{T}: \mathcal{M} \rightarrow \mathcal{M}$ acting by formulae (A.I.1)-(A.I.5). Again using the arguments applied in the proof of (3.2) and, in particular, the estimate (3.5), we find that the norm of the operator $\mathcal{T}$ is bounded above:

$$
\|\mathcal{T}\|<e\left(\frac{B_{1}}{\lambda_{2}^{0}(P)-z}+B_{2}\right)
$$

where $B_{1}>0, B_{2}>0$ are constants. From this it is clear that for any $\varepsilon>0$ and for sufficiently small values of $e$ the norm satisfies $\|\mathcal{T}\|<1$. Thus, if the components of the vector $F_{2}$ that is a solution of the equation

$$
F_{2}+Q(z) F_{2}=G(z) F_{1}
$$

are represented in the form (3.24), then the tuple of coefficient functions

$$
\mu=\left\{b_{10}, b_{01}, d_{10}, d_{01}, e_{00}\right\}
$$

involved in this representation must satisfy the equation

$$
\mu+\mathcal{T} \mu=\mu^{0} .
$$

For sufficiently small $e$ this equation has a unique solution in the space $\mathcal{M}$ and its norm is bounded above:

$$
\|\mu\|<e\left(\frac{D_{1}}{\lambda_{2}^{0}(P)-z}+D_{2}\right),
$$

where $D_{1}>0, D_{2}>0$ are constants. We have thus proved the representation (3.24) and established the estimates (3.25) for the coefficient functions themselves. The estimates for the derivatives of these functions with respect to their arguments can be obtained by the recurrence procedure described in 1]. Namely, by differentiating equation (A.I.13), 
say, with respect to the first argument $q_{1} \in \gamma_{1}$, we obtain that the derivatives $\nabla_{q_{1}} \mu$ again satisfy equation (A.I.13) with a modified right-hand side containing functions in the tuple $\mu$. Thus, $\nabla_{q} \mu$ exists and admits an estimate of the form (3.25). By continuing to differentiate further, by degrees we obtain all the derivatives up to order $r$ and obtain their estimates (3.25).

For a detailed exposition, see 1 .

II. Proof of Lemma 4.1. Consider the operator

$$
V=D(M-z \widetilde{\mathbb{I}})^{-1},
$$

where $D$ is the operator in $\mathcal{H}_{1}$ with matrix kernel $D\left(q, q^{\prime} ; \xi\right)$ and $M$ is the operator of multiplication by the matrix function $M(q ; \xi)$ (see (4.1)). We can formally represent the resolvent $R_{B(\xi)}$ as the series

$$
R_{B(\xi)}=\left(M-w I_{1}\right)^{-1}+\sum_{n=1}^{\infty}(-1)^{n}\left(M-w I_{1}\right)^{-1} V^{n},
$$

where $\left(M-w I_{1}\right)^{-1} V^{n}, n \geq 1$, is an integral operator with kernel

$$
\left(a_{P, 1}(q, \xi)-w\right)^{-1} L_{n}\left(q, q^{\prime} ; \xi, w\right)\left(a_{P, 1}(q, \xi)-w\right)^{-1},
$$

where $L_{n}\left(q, q^{\prime} ; \xi, w\right)$ is the matrix kernel equal to

$$
L_{n}\left(q, q^{\prime} ; \xi, w\right)=\int \ldots \int \frac{D\left(q, q_{1} ; \xi\right) D\left(q, q_{2} ; \xi\right) \cdots D\left(q_{n-1}, q^{\prime} ; \xi\right)}{\prod_{i=1}^{n-1}\left(a_{P, 1}\left(q_{i}, \xi\right)-w\right)} d q_{1} \cdots d q_{n-1} .
$$

We must verify the following properties of the kernels $L_{n}\left(q, q^{\prime} ; \xi, w\right)$ :

i) $L_{n}\left(q, q^{\prime} ; \xi, w\right) \in \mathcal{B}$ and $\left\|L_{n}\right\|_{\mathcal{B}}<\left(C e^{2}\right)^{n-1}$, where $C$ is a constant;

ii) $\lim _{\varepsilon \backslash 0} L_{n}\left(q, q^{\prime} ; \xi ; x \pm i \varepsilon\right)=L_{n}\left(q, q^{\prime} ; \xi ; x\right), x \in \mathcal{I}(\xi)$, exists in the space $\mathcal{B}$ for all $\mathcal{I}(\xi)$

iii) $L_{n}^{ \pm}\left(q, q^{\prime} ; \xi ; x\right)$ as a $\mathcal{B}$-valued function of $x \in \mathcal{I}(\xi)$ satisfies the Hölder condition with exponent $1 / 3$ (see (4.5)), that is,

$$
\max _{i, j}\left|\partial_{q}^{\alpha} \partial_{q}^{\alpha^{\prime}} L_{n, i, j}^{ \pm}\left(q, q^{\prime} ; \xi ; x\right)\right|<N h(q) h\left(q^{\prime}\right) \quad \text { for all } \quad|\alpha| ;\left|\alpha^{\prime}\right|<3
$$

and

$\left|\partial_{q}^{\alpha} \partial_{q^{\prime}}^{\alpha^{\prime}} L_{n, i, j}^{ \pm}\left(q, q^{\prime} ; \xi ; x+\Delta\right)-\partial_{q}^{\alpha} \partial_{q^{\prime}}^{\alpha^{\prime}} L_{n, i, j}^{ \pm}\left(q, q^{\prime} ; \xi ; x\right)\right|<N h(q) h\left(q^{\prime}\right) \Delta^{1 / 3}, \quad 0<\Delta<1$, where $N=(C e)^{n-1}$.

These properties of the kernels $L_{n}$ obviously ensure that the series (A.II.2) converges, the limit (4.4) exists and that property (4.5) holds. We verify i), ii), iii) by induction. For $n=1$, when $L_{1}\left(q, q^{\prime}, \xi, w\right)=D\left(q, q^{\prime} ; \xi\right)$, all these properties are obvious and follow from the smoothness of $D\left(q, q^{\prime} ; \xi\right)$ (see (3.35) ). For $\operatorname{Im} w>0$ we have

$$
\begin{aligned}
L_{n+1}\left(q, q^{\prime} ; \xi, w\right) & =\int D\left(q, \bar{q}^{\prime} ; \xi\right) L_{n}\left(\bar{q}, q^{\prime} ; \xi, w\right)\left(a_{P, 1}(\bar{q} ; \xi)-w\right)^{-1} d \bar{q} \\
& =i \int d t e^{i w t} \int D\left(q, \bar{q}^{\prime} ; \xi\right) L_{n}\left(\bar{q}, q^{\prime} ; \xi, w\right) e^{-i t a_{P, 1}(\bar{q} ; \xi)} d \bar{q}
\end{aligned}
$$

and, consequently,

$$
\begin{aligned}
& \partial_{q}^{\alpha} \partial_{q^{\prime}}^{\alpha^{\prime}} L_{n+1}\left(q, q^{\prime} ; \xi, w\right) \\
& \quad=i \int_{0}^{\infty} e^{i w t} d t \int \partial_{q}^{\alpha} D(q, \bar{q} ; \xi) \partial_{q^{\prime}}^{\alpha^{\prime}} L_{n}\left(\bar{q}, q^{\prime} ; \xi, w\right) e^{-i t a_{P, 1}(\bar{q} ; \xi)} d \bar{q} .
\end{aligned}
$$


Using (3.35) and the induction hypothesis, we can represent the inner integral in (A.II.5) by the stationary phase method (see [3]) in the form

$$
\frac{\widehat{C} \partial_{q}^{\alpha} D\left(q, q_{0} ; \xi\right) \partial_{q^{\prime}}^{\alpha^{\prime}} L_{n}\left(q_{0}, q^{\prime} ; \xi, w\right) e^{-i t a_{P, 1}\left(q_{0} ; \xi\right)}}{t^{3 / 2}+1}+\Delta_{\alpha, \alpha^{\prime}}\left(t, q, q^{\prime}\right),
$$

where $q_{0}$ is a minimum point of the function $a_{P, 1}(q ; \xi), \widehat{C}$ is an absolute constant, and the matrix kernel $\Delta_{\alpha, \alpha^{\prime}}\left(t, q, q^{\prime}\right)$ satisfies the estimate

$$
\max _{i, j}\left|\Delta_{\alpha, \alpha^{\prime}}^{i, j}\right|<\bar{N}(C e)^{n-1} \frac{h(q) h\left(q^{\prime}\right)}{t^{5 / 2}+1},
$$

where $\bar{N}$ is a constant. Thus, since $\partial_{q}^{\alpha} D\left(q, q^{\prime}\right) \sim e$, the contribution to (A.II.4) of each of the summands in (A.II.6) is bounded above by

$$
\widehat{C} \bar{N}(C e)^{n} h(q) h\left(q^{\prime}\right) .
$$

Next, for $\operatorname{Im} w \geq 0$ the integral

$$
\int_{0}^{\infty} \frac{d t e^{i\left(w-a_{P, 1}\left(q_{0} ; \xi\right)\right) t}}{t^{3 / 2}+1}
$$

is a bounded continuous function of $w$ satisfying a Hölder condition with exponent $1 / 2$; the contribution of the first summand in (A.II.6) also has this property. By (A.II.7), the same is true for the contribution of the second summand in (A.II.6). Lemma 4.1 is proved.

III. Proof of Lemma 4.2, 1. Since

$$
a_{00}(\xi)-\Delta^{-}\left(a_{P, 1}(\bar{q} ; \xi)\right)-a_{P, 1}(\bar{q} ; \xi) \neq 0
$$

for all $\bar{q}$, we need to verify that the integral

$$
\int \frac{\left(K^{-}\left(q^{\prime}, \bar{q} ; \xi ; a_{P, 1}(\bar{q} ; \xi)\right) s^{\alpha}, v\left(q^{\prime}\right)\right)}{a_{P, 1}\left(q^{\prime} ; \xi\right)-a_{P, 1}(\bar{q} ; \xi)+i 0} d q^{\prime},
$$

which can be represented in the form

$$
i \int_{0}^{\infty} d t \int e^{i t\left(a_{P, 1}\left(q^{\prime} ; \xi\right)-a_{P, 1}(\bar{q} ; \xi)\right)}\left(K^{-}\left(q^{\prime}, \bar{q} ; \xi ; a_{P, 1}(\bar{q} ; \xi)\right) s^{\alpha}, v\left(q^{\prime}\right)\right) d q^{\prime},
$$

is continuous and bounded, and again apply the stationary phase method, as we did in the proof of the preceding lemma.

2. The first assertion is obvious. In order to prove the second assertion for each fixed $q$ we consider the integral

$$
C_{10, \varphi}^{\alpha}=\int f_{10, \bar{q}}^{\alpha} \varphi(\bar{q}) d \bar{q},
$$

where $\varphi \in S\left(R^{3}\right)$. The first two summands in (4.19), as functions of $\bar{q}$, obviously belong to $S^{\prime}\left(R^{3}\right)$ and their contribution to the integral (A.III.2) is well defined. We consider the final, "suspicious" summand in (4.19). It can be represented in the form

$$
\int m_{\varphi}^{\alpha}(x) I^{\alpha}(x, q) d x
$$

where

$$
I^{\alpha}(x, q)=i \int_{0}^{\infty} d t \int e^{i t\left(a_{P, 1}\left(q^{\prime} ; \xi\right)-x\right)}\left(K^{-}\left(q, q^{\prime} ; \xi, x\right), v\left(q^{\prime}\right)\right) d q^{\prime}
$$

and

$$
m_{\varphi}^{\alpha}(x)=\int_{a_{P, 1}(\bar{q}, \xi)=x} f_{00, \bar{q}}^{\alpha} \varphi(\bar{q}) d \bar{q} .
$$


Again using the stationary phase method we can verify that $I^{\alpha}(x, q)$ is a bounded (Hölder) function of $x \in \mathcal{I}(\xi)$. Since the critical point of the function $a_{P, 1}(\bar{q}, \xi)$ is nondegenerate, the function $m_{\varphi}^{\alpha}(x)$ is a bounded (Hölder) function of $x \in \mathcal{I}(\xi)$, which, since $\varphi(q)$ decreases rapidly as $q \rightarrow \infty$, is also rapidly decreasing as $x \rightarrow \infty$. Thus, the integral (A.III.3) is well-defined (and depends continuously on $\varphi$ in the topology of $S\left(R^{3}\right)$ ).

3. We use the representation

$$
\varphi(\bar{q})=\int \varphi\left(q_{0}\right) \delta\left(\bar{q}-q_{0}\right) d q_{0},
$$

from which we find that

$$
W(\xi)\left(\varphi s^{\alpha}\right)=\int \varphi\left(q_{0}\right) F_{q_{0}}^{\alpha} d q_{0}
$$

Since $W(\xi): \mathcal{H}^{1} \rightarrow \mathcal{H}_{a c}$ is unitary, we have the equality

$$
\begin{aligned}
&\left(\varphi_{1} s^{\alpha_{1}}, \varphi_{2} s^{\alpha_{2}}\right)_{\mathcal{H}^{1}}=\delta_{\alpha_{1} \alpha_{2}}\left(\varphi_{1}, \varphi_{2}\right)_{L_{2}\left(R^{3}\right)}=\left(\psi_{\varphi_{1}}^{\alpha_{1}}, \psi_{\varphi_{2}}^{\alpha_{2}}\right) \\
&=C_{00, \varphi_{1}}^{\alpha_{1}} \cdot \bar{C}_{00, \varphi_{2}}^{\alpha_{2}}+\int\left(C_{10, \varphi_{1}}^{\alpha_{1}}(q) \cdot \bar{C}_{10, \varphi_{2}}^{\alpha_{2}}(q)+C_{01, \varphi_{1}}^{\alpha_{1}}(q) \cdot \bar{C}_{01, \varphi_{2}}^{\alpha_{2}}(q)\right) d q,
\end{aligned}
$$

that is, equality (4.20). Lemma 4.2 is proved.

IV. Proof of Lemma 5.1. The first assertion of the lemma follows from the fact that the function $a_{00}(\xi)-\Delta^{-1}(x)-x$ is separated from zero for $x \in \mathcal{I}(\xi)$, the estimate (3.34) for $v_{\alpha}$, and estimates for the integrals in (4.18) and (4.19) obtained by using their representations, which are similar to (A.II.4), and then applying the stationary phase method.

The second and third assertions of the lemma follow from the estimates (3.25) for the coefficient functions and from the properties of the lower components $f_{00, \bar{q}}^{\alpha}, f_{10, \bar{q}}^{\alpha}, f_{01, \bar{q}}^{\alpha}$ of the vector $F_{\bar{q}}^{\alpha}$ proved above. See [1] for the details.

\section{REFERENCES}

[1] N. Angelescu, R. A. Minlos, and V. A. Zagrebnov, Lower spectral branches of a particle coupled to a Bose field, Rev. Math. Phys. 17 (2005), no. 9, 1-32. MR2187291 (2007b:81072)

[2] A. Arai, Spectral analysis of a quantum harmonic oscillator coupled to infinitely many scalar bosons, J. Math. Anal. Appl. 140 (1989), 270-288. MR0997857 (90j:81035)

[3] V.I. Arnol'd, S. M. Guser̆n-Zade, and A. N. Varchenko, Singularities of differentiable maps. Vol. II. Monodromy and asymptotics of integrals, Nauka, Moscow, 1984; English transl., Birkhäuser, Boston, MA, 1988. MR0966191 (89g:58024)

[4] V. Bach, J. Fröhlich, and A. Pizzo, Infrared-finite algorithms in QED: the groundstate in an atom interacting with the quantized radiation field, Commun. Math. Phys. 264 (2006), no. 1, 145-165. MR2212219(2007d:81248)

[5] V. Bach, J. Fröhlich, and I. M. Sigal,

1. Spectral analysis for systems of atoms and molecules coupled to the quantized radiation field, Commun. Math. Phys. 207 (1999), no. 2, 249-290. MR1724854 (2001j:81256)

2. Quantum electrodynamics of confined nonrelativistic particles, Adv. Math. 137 (1998), no. 2, 299-395. MR1639713 (99e:81051b)

[6] Ju. M. Berezanskiū, Expansions in eigenfunctions of selfadjoint operators, Naukova Dumka, Kiev, 1965; English transl., Translations of Mathematical Monographs, Vol. 17, Amer. Math. Soc., Providence, RI, 1968. MR0222718 (36:5768)

[7] F. A. Berezin, The method of second quantization, Nauka, Moscow, 1965; English transl., Pure and Applied Physics, Vol. 24 Academic Press, New York, 1966. MR0208930 (34:8738)

[8] M.Sh. Birman and M.Z. Solomyak, Spectral theory of selfadjoint operators in Hilbert space, Leningrad Univ., Leningrad, 1980; English transl., Mathematics and its Applications (Soviet Series). D. Reidel, Dordrecht, 1987. MR.1192782 (93g:47001)

[9] J. Dereziński and C. Gérard, Asymptotic completeness in quantum field theory. Massive Pauli-Fierz Hamiltonians, Rev. Math. Phys. 11 (1999), no. 4, 383-450. MR1682684 (2000h:81141)

[10] H. Fröhlich, Electrons in lattice fields, Adv. Phys. 3 (1954), 325-364. 
[11] J. Fröhlich,

1. On the infrared problem in a model of scalar electrons and massless, scalar bosons, Ann. Inst. H. Poincaré Sect. A (N. S.) 19 (1973), 1-103. MR0368649 (51:4890)

2. Existence of dressed one-electron states in a class of persistent models, Fortschr. Phys. 22 (1974), 159-198.

[12] J. Fröhlich, M. Griesemer, and B. Schlein, Asymptotic completeness for Compton scattering, Commun. Math. Phys. 252 (2004), no. 1-3, 415-476. MR2104885 (2006d:81331)

[13] C. Gérard,

1. On the existence of ground states for massless Pauli-Fierz Hamiltonians, Ann. Henri Poincaré 1 (2000), no. 3, 443-459. MR.1777307 (2001g:81065)

2. On the scattering theory of massless Nelson models, Rev. Math. Phys. 14 (2002), no. 11, 1165-1280. MR.1943190 (2004g:81137)

[14] I. M. Gel'fand and G. E. Shilov, Generalized functions, Vol. 3, Nauka, Moscow, 1984; English transl. of 1st ed.: Generalized functions. Vol. 3: Theory of differential equations, Academic Press, New York, 1967. MR0217416 (36:506)

[15] B. Gerlach and H. Löwen, Analytical properties of polaron systems or: Do polaronic phase transitions exist or not? Rev. Modern Phys. 63 (1991), no. 1, 63-90. MR.1102193 (92j:82021)

[16] M. Hirokawa, F. Hiroshima, and H. Spohn, Ground state for point particles interacting through a massless scalar Bose field, Adv. Math. 191 (2005), no. 2, 339-392. MR.2103217 (2005h:81206)

[17] F. Hiroshima and H. Spohn, Ground state degeneracy of the Pauli-Fierz Hamiltonian with spin, Adv. Theor. Math. Phys. 5 (2001), no. 6, 1091-1104. MR1926665 (2003m:81294)

[18] F. Hiroshima, Ground states and spectrum of quantum electrodynamics of nonrelativistic particles, Trans. Amer. Math. Soc. 353 (2001), no. 11, 4497-4528 (electronic). MR1851181 (2002f:81014)

[19] T. D. Lee, F. E. Low, and D. Pines, The motion of slow electrons in a polar crystal, Phys. Rev. (2) 90 (1953) 297-302. MR0103072 (21:1856)

[20] E. H. Lieb and L. E. Thomas, Exact ground state energy of the strong-coupling polaron, Commun. Math. Phys. 183 (1997), no. 3, 511-519; Erratum: "Exact ground state energy of the strong-coupling polaron", ibid. 188 (1997), no. 2, 499-500. MR1462224(99a:81034a) MR.1471825(99a:81034b)

[21] V.A. Malyshev and R. A. Minlos, Linear operators in infinite-particle systems, Nauka, Moscow, 1994; English transl.: Linear infinite-particle operators, Translations of Mathematical Monographs, Vol. 143, Amer. Math. Soc., Providence, RI, 1995. MR1317349 (96d:82038)

[22] R. A. Minlos, On the lower branch of the spectrum of a fermion interacting with a boson gas (a polaron), Teoret. Mat. Fiz. 92 (1992), no. 2, 255-268; English transl. in Theoret. Math. Phys. 92 (1993), no. 2, 869-878. MR1226014 (94b:81153)

[23] J.S. Møller, The translation invariant massive Nelson model. I. The bottom of the spectrum, Ann. Henri Poincaré 6 (2005), no. 6, 1091-1135. MR2189378 (2006m:81168)

[24] M. Reed and B. Simon, Methods of modern mathematical physics. III. Scattering theory, Academic Press, New York, 1979; IV. Analysis of operators, Academic Press, New York, 1978. MR0529429 (80m:81085) MR0493421 (58:12429c)

[25] H. Spohn,

1. Ground state of a quantum particle coupled to a scalar Bose field, Lett. Math. Phys. 44 (1998), no. 1, 9-16. MR.1623746 (99c:81278)

2. The polaron at large total momentum, J. Phys. A 21 (1988), no. 5, 1199-1211. MR0939707 (89m:81207)

3. Dynamics of charged particles and their radiation field, Cambridge Univ. Press, Cambridge, 2004. MR2097788 (2005g:81355)

[26] D. R. Yafaev, Mathematical scattering theory. General theory, S.-Peterburg. Univ., 1994; English transl., Transl. of Math. Monographs, Vol. 105. Amer. Math. Soc., Providence, RI, 1992. MR.1180965 (94f:47012)

Institute for Information Transmission Problems, Russian Academy of Sciences, Moscow, Russia

E-mail address: minl@iitp.ru

Translated by E. KHUKHRO 